\title{
Overview of methods for deriving the radiative transfer theory from the Maxwell equations. I: Approach based on the far-field Foldy equations
}

\author{
Adrian Doicu $^{\mathrm{a}, *}$, Michael I. Mishchenko ${ }^{\mathrm{b}}$ \\ a Deutsches Zentrum für Luft- und Raumfahrt (DLR), Institut für Methodik der Fernerkundung (IMF), Oberpfaffenhofen 82234, Germany \\ ${ }^{\mathrm{b}}$ NASA Goddard Institute for Space Studies, 2880 Broadway, New York, NY 10025, USA
}

\section{A R T I C L E I N F O}

\section{Article history:}

Received 30 July 2018

Revised 5 September 2018

Accepted 5 September 2018

Available online 7 September 2018

\section{Keywords:}

Electromagnetic scattering

Frequency-domain macroscopic

electromagnetics

Discrete random media

Foldy equations

Ladder diagrams

Radiative transfer theory

\begin{abstract}
A B S T R A C T
In this paper, we revisit, with further enhancements and clarifications, the self-consistent first-principles approach developed previously for deriving the vector radiative transfer theory for a discrete random medium with a sparse concentration of particles. We specifically consider the case of a plane-parallel particulate layer embedded in an otherwise homogeneous unbounded medium. The solution method is based on the far-field Foldy equations, an order-of-scattering expansion for the total field derived under the Twersky approximation, the computation of the coherent field by assuming that the positions of the particles are uncorrelated, and the ladder approximation for the coherency dyadic. The latter yields an integral equation for the diffuse specific coherency dyadic, defined through an angular spectrum representation for the coherency dyadic, which in turn, gives the vector radiative transfer equation for the diffuse specific intensity column vector. We analyze specifically the computation of the coherent field for inhomogeneous particulate media and multiple species of particles, the continuous extension of the far-field representation to the near field, the Foldy approximation, and the Foldy integral equation for the coherent field. Finally, we discuss the transition from the vector to the scalar radiative transfer equation.
\end{abstract}

(c) 2018 Elsevier Ltd. All rights reserved.

\section{Introduction}

In a series of papers we aim to analyze several methods for deriving the radiative transfer theory for a discrete random layer with a sparse concentration of particles from the macroscopic Maxwell equations. For such media we make the following generic assumptions adhered to in most cases:

1. The positions of the particles are uncorrelated (statistically independent), and the spatial distribution of the particles is statistically uniform.

2. Each particle is located in the far-field regions of all the other particles, and the observation point is also located in the farfield region of any particle (but in the near-field region of the entire group).

In the first two papers, we consider non-spherical particles distributed in a domain confined to a layer with non-scattering boundaries, while in the third paper, we consider spherical particles distributed in a layer with scattering boundaries. Roughly

\footnotetext{
* Corresponding author.

E-mail address: adrian.doicu@dlr.de (A. Doicu).
}

speaking, the solution methods rely on (i) the far-field Foldy equations obtained under the far-field approximation, (ii) the Dyson and the Bethe-Salpeter equations derived from the Foldy equations, and (iii) the Wigner transform of the Bethe-Salpeter equation for the dyadic correlation function. Our overarching goal is to analyze whether the different methods give consistent results, as well as to emphasize the similarities and differences between the underlying assumptions.

The first method to be discussed is the self-consistent firstprinciples approach for addressing the scattering by sparse discrete random media developed in Refs. [1-4]. The main advantage of this approach is that the statistical properties of the radiation field are not postulated but rather follow from specific underlying assumptions about the micro- and macrophysical properties of the random particulate medium. Furthermore, this approach addresses explicitly the two fundamental problems of macroscopic electromagnetics as applied to discrete random media: (i) how to quantify the reading of a specific detector of electromagnetic energy flow called a well-collimated radiometer, and (ii) how to quantify the energy budget of a finite volume of particulate medium [3,4]. As a consequence, all primary and derivative optical observables (such as the Poynting-Stokes dyad, the dyadic correlation function, the 


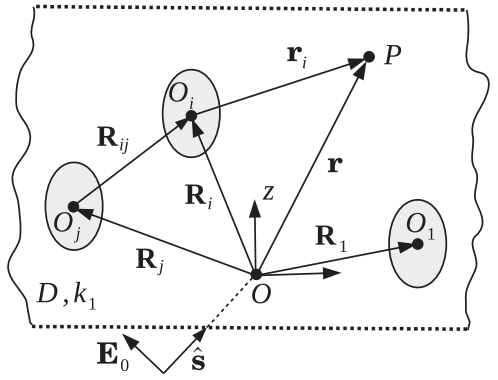

(a)

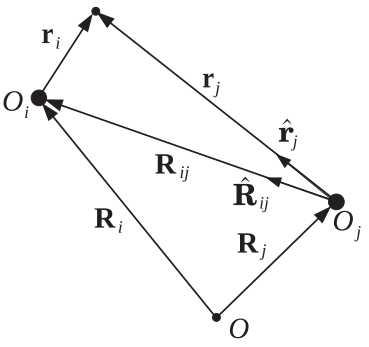

(b)
Fig. 1. (a) A system of particles confined to a layer with non-scattering boundaries, and (b) the geometry for computing the field exciting particle $i$ due to particle $j$.

coherent and diffuse specific intensity column vectors, etc.) emerge naturally and have a well-defined physical and practical meaning.

Below we supplement the summary of this approach by several mathematical enhancements and clarifications. In particular, we analyze the computation of the coherent field for inhomogeneous particulate media and multiple species of particles, the continuous extension of the far-field representation to the near field, the Foldy approximation, and the Foldy integral equation for the coherent field. We also outline an approach to derive the scalar radiative transfer theory.

\section{Scattering problem}

We consider a discrete random medium [5] in the form of a group of $N$ identical particles centered at $\mathbf{R}_{1}, \mathbf{R}_{2}, \ldots, \mathbf{R}_{N}$, and distributed in a domain $D$ confined to a laterally infinite plane-parallel layer with imaginary (non-scattering) boundaries (Fig. 1(a)). For simplicity, we assume that the particles are homogeneous, have the same orientation, and that the particle coordinate systems are aligned with the global coordinate system. The permittivities of the nonabsorbing background medium and the particles are $\varepsilon_{1}$ and $\varepsilon_{2}$, respectively, while the wavenumbers are $k_{1}$ and $k_{2}=\mathrm{m} k_{1}$, where $\mathrm{m}$ is the relative refractive index of the particles. The discrete medium is characterized by the number concentration (the number of particles per unit volume) $n_{0}=N / V$, where $V$ is the volume occupied by the particles. If $n_{0}$ is small then the particulate medium is sparse; otherwise the medium is dense. Because the medium is infinite in the horizontal directions, we let $V \rightarrow \infty$ and $N \rightarrow \infty$ such that $n_{0}$ is constant. The particulate layer is illuminated by a plane electromagnetic wave with propagation direction $\widehat{\mathbf{s}}$ and amplitude $\mathcal{E}_{0}(\widehat{\mathbf{s}})$,

$\mathbf{E}_{0}(\mathbf{r}, t)=\mathbf{E}_{0}(\mathbf{r}) \mathrm{e}^{-\mathrm{j} \omega \mathrm{t}}, \mathbf{E}_{0}(\mathbf{r})=\mathcal{E}_{0}(\widehat{\mathbf{s}}) \mathrm{e}^{\mathrm{j} \mathrm{k}_{1} \widehat{\mathbf{s}} \cdot \mathbf{r}}, \widehat{\mathbf{s}} \cdot \mathcal{E}_{0}(\widehat{\mathbf{s}})=0$,

where $\mathrm{j}=\sqrt{-1}, t$ is time, and $\omega$ is the angular frequency. The harmonic time dependence will be implicit form now on.

\section{Optical observables and assumption of ergodicity}

In the discipline of electromagnetic scattering by particles, the computation of the scattered field in and of itself is usually not the end goal since in most cases this field is not directly observable, especially in the optical and infrared range of frequencies [6]. Furthermore, any actual measurement takes a finite amount of time over which any first moment in the electromagnetic field would vanish as a result of an extremely high frequency of timeharmonic oscillations. One must therefore be careful in defining relevant optical observables as measurable second moments in the field such that they do not vanish upon temporal averaging. Another key aspect is the presumed temporal randomness of the particulate medium. The computation of the instantaneous scattered field followed by averaging over time of an optical observable represent an exceedingly difficult problem since they require the invocation of an explicit dynamical model of the medium. We will deal with this aspect by assuming full ergodicity of the random multi-particle group and replacing temporal averaging by ensemble averaging $[2,3]$. However, it is imperative to recognize that ensemble averaging is not a primordial physical concept and can only be used as a substitute for time averaging. As summarized eloquently by Truesdell [7], "The purpose of statistical mechanics, for phenomena in equilibrium, is to calculate time averages, and the ensemble theory is useful only as a tool enabling us to calculate time averages without knowing how to integrate the equations of motion. The ensemble theory is a mathematical device; we are wasting our time if we try to explain it by itself."

Before proceeding we give a brief summary of the probability theory needed. Let $\Lambda_{N}=\left\{\mathbf{R}_{1}, \ldots, \mathbf{R}_{N}\right\}$ be a spatial configuration of $N$ particles, and $p\left(\Lambda_{N}\right) \mathrm{d} \Lambda_{N}$ be the probability $p\left(\mathbf{R}_{1}, \ldots, \mathbf{R}_{N}\right) \mathrm{d}^{3} \mathbf{R}_{1} \ldots \mathrm{d}^{3} \mathbf{R}_{N}$ of finding the particles in a configuration in which the first particle resides in the volume element $\mathrm{d}^{3} \mathbf{R}_{1}$ centered at the point $\mathbf{R}_{1}$, the second particle resides in the volume element $d^{3} \mathbf{R}_{2}$ centered at the point $\mathbf{R}_{2}$, and so on up to $\mathbf{R}_{N}$. We assume that the integral of the joint probability density function $p\left(\Lambda_{N}\right)=p\left(\mathbf{R}_{1}, \ldots, \mathbf{R}_{N}\right)$ over all configurations is normalized to unity, and that the $N$ particles are indistinguishable, so that the order of the arguments of $p$ is irrelevant. According to the Bayes theorem, we have

$$
\begin{aligned}
p\left(\Lambda_{N}\right) & =p\left(\mathbf{R}_{i}\right) p\left(\Lambda_{N-1}^{i} \mid \mathbf{R}_{i}\right) \\
& =p\left(\mathbf{R}_{i}\right) p\left(\mathbf{R}_{j} \mid \mathbf{R}_{i}\right) p\left(\Lambda_{N-2}^{i j} \mid \mathbf{R}_{i}, \mathbf{R}_{j}\right),
\end{aligned}
$$

where $p\left(\mathbf{R}_{i}\right)$ is the probability of finding particle $i$ at the point $\mathbf{R}_{i}, p\left(\Lambda_{N-1}^{i} \mid \mathbf{R}_{i}\right)$ with $\Lambda_{N-1}^{i}=\Lambda_{N} \backslash\left\{\mathbf{R}_{i}\right\}$ is the conditional probability of finding the remaining particles at the corresponding points, $p\left(\mathbf{R}_{j} \mid \mathbf{R}_{i}\right)$ is the conditional probability of finding particle $j$ at the point $\mathbf{R}_{j}$ if it is known that particle $i$ is at the point $\mathbf{R}_{i}$, and $p\left(\Lambda_{N-2}^{i j} \mid \mathbf{R}_{i}, \mathbf{R}_{j}\right)$ with $\Lambda_{N-2}^{i j}=\Lambda_{N} \backslash\left\{\mathbf{R}_{i}, \mathbf{R}_{j}\right\}$ is the conditional probability of finding the remaining particles at the corresponding points. Note that $p\left(\Lambda_{N}\right)$ can also be expressed in terms of joint probability density functions, e.g.,

$p\left(\mathbf{R}_{i}, \mathbf{R}_{j}\right)=p\left(\mathbf{R}_{i}\right) p\left(\mathbf{R}_{j} \mid \mathbf{R}_{i}\right)$,

where $p\left(\mathbf{R}_{i}, \mathbf{R}_{j}\right) \mathrm{d}^{3} \mathbf{R}_{i} \mathrm{~d}^{3} \mathbf{R}_{j}$ is the joint probability of finding particle $i$ in $\mathrm{d}^{3} \mathbf{R}_{i}$ and particle $j$ in $\mathrm{d}^{3} \mathbf{R}_{j}$. The configuration average of a function $f\left(\mathbf{r}, \Lambda_{N}\right)$ is given by

$$
\begin{aligned}
\left\langle f\left(\mathbf{r}, \Lambda_{N}\right)\right\rangle & =\int f\left(\mathbf{r}, \Lambda_{N}\right) p\left(\Lambda_{N}\right) \mathrm{d} \Lambda_{N} \\
& =\int f\left(\mathbf{r}, \Lambda_{N}\right) p\left(\mathbf{R}_{i}\right) p\left(\Lambda_{N-1}^{i} \mid \mathbf{R}_{i}\right) \mathrm{d} \Lambda_{N-1}^{i} \mathrm{~d}^{3} \mathbf{R}_{i} \\
& =\int p\left(\mathbf{R}_{i}\right)\left\langle f\left(\mathbf{r}, \Lambda_{N}\right)\right\rangle_{i} \mathrm{~d}^{3} \mathbf{R}_{i},
\end{aligned}
$$

where

$\left\langle f\left(\mathbf{r}, \Lambda_{N}\right)\right\rangle_{i}=\int f\left(\mathbf{r}, \Lambda_{N}\right) p\left(\Lambda_{N-1}^{i} \mid \mathbf{R}_{i}\right) \mathrm{d} \Lambda_{N-1}^{i}$

is the conditional configuration average of $f\left(\mathbf{r}, \Lambda_{N}\right)$ while holding the position of particle $i$ fixed. In particular, if the positions of the particles are statistically independent (i.e., their positions are uncorrelated), we have, for example, $p\left(\mathbf{R}_{j} \mid \mathbf{R}_{i}\right)=p\left(\mathbf{R}_{j}\right)$, and so,

$$
p\left(\Lambda_{N}\right)=p\left(\mathbf{R}_{i}\right) p\left(\Lambda_{N-1}^{i}\right)=p\left(\mathbf{R}_{i}\right) p\left(\mathbf{R}_{j}\right) p\left(\Lambda_{N-2}^{i j}\right)=\ldots=\prod_{i} p\left(\mathbf{R}_{i}\right)
$$

where the multiplication runs implicitly from $i=1$ to $i=N$. Moreover, if the particles are distributed uniformly throughout the scattering layer then the positions of all the particles are equally 
probable within the volume $V$, and the single particle probability density function is

$p\left(\mathbf{R}_{i}\right)=\frac{1}{V}$.

Consequently, if the positions of the particles are independent and the particles are uniformly distributed, the configuration average of the sum $\Sigma_{i} f\left(\mathbf{r}, \mathbf{R}_{i}\right)$ computes as

$\sum_{i}\left\langle f\left(\mathbf{r}, \mathbf{R}_{i}\right)\right\rangle=N \int f\left(\mathbf{r}, \mathbf{R}_{i}\right) p\left(\mathbf{R}_{i}\right) \mathrm{d}^{3} \mathbf{R}_{i}=n_{0} \int f\left(\mathbf{r}, \mathbf{R}_{i}\right) \mathrm{d}^{3} \mathbf{R}_{i}$,

where, again, the summation runs implicitly from $i=1$ to $i=N$.

To simplify the statement about the assumptions that we use, we adopt the following convention: when we say that the positions of the particles are statistically independent, we also mean that the particles are uniformly distributed. Thus, $p\left(\Lambda_{N}\right)$ is given by Eq. (6) in conjunction with Eq. (7).

\section{Far-field Foldy equations}

From now on, we will assume that (i) each particle is located in the far zones of all the other particles, and (ii) the observation point is located in the far zones of all the particles forming the particulate layer. Note that since the particulate medium is infinite, the observation point always resides in the near field of the medium.

The total field at position $\mathbf{r}$ sums the contribution of the incident field and of the fields scattered by all particles

$\mathbf{E}(\mathbf{r})=\mathbf{E}_{0}(\mathbf{r})+\sum_{i} \mathbf{E}_{\mathrm{scti}}(\mathbf{r})$.

The field exciting particle $i, \mathbf{E}_{\text {exci }}^{(i)}\left(\mathbf{r}_{i}\right)$, is a superposition of the incident field $\mathbf{E}_{0}^{(i)}\left(\mathbf{r}_{i}\right)$ and of the exciting fields $\mathbf{E}_{\text {excij }}^{(i)}$ produced by all particles $j$ excepting $i$, that is,

$\mathbf{E}_{\text {exci }}^{(i)}\left(\mathbf{r}_{i}\right)=\mathbf{E}_{0}^{(i)}\left(\mathbf{r}_{i}\right)+\sum_{j \neq i} \mathbf{E}_{\text {excij }}^{(i)}\left(\mathbf{r}_{i}\right)$.

Here, the superscript $(i)$ indicates that the fields are written in the coordinate system of particle $i$.

In the far-field region, the field scattered by particle $i$ is

$\mathbf{E}_{\mathrm{sct} i}(\mathbf{r})=\mathbf{E}_{\mathrm{sct} i}^{(i)}\left(\mathbf{r}_{i}\right)=\frac{\mathrm{e}^{\mathrm{j} \mathrm{k}_{1} \mathrm{r}_{\mathrm{i}}}}{r_{i}} \mathbf{E}_{\mathrm{sct} i}^{\infty}\left(\widehat{\mathbf{r}}_{i}\right), \quad r_{i} \rightarrow \infty$,

where $\mathbf{E}_{\mathrm{sct} i}^{\infty}\left(\widehat{\mathbf{r}}_{i}\right)$ is the (electric) far-field pattern. Using the approximation $\widehat{\mathbf{r}}_{j} \approx \widehat{\mathbf{R}}_{i j}$, implying (Fig. 1(b))

$\frac{\mathrm{e}^{\mathrm{j} \mathrm{k}_{1} \mathrm{r}_{\mathrm{j}}}}{r_{j}} \approx \frac{\mathrm{e}^{\mathrm{j} \mathrm{k}_{1} \mathrm{R}_{\mathrm{ij}}}}{R_{i j}} \mathrm{e}^{\mathrm{j} \mathrm{k}_{1} \widehat{\mathbf{R}}_{\mathrm{ij}} \cdot \mathbf{r}_{\mathrm{i}}}$,

we find that locally, the contribution of the $j$ th particle to the field exciting particle $i$ is a plane electromagnetic wave of amplitude $\mathbf{E}_{i j}$ propagating in the direction $\widehat{\mathbf{R}}_{i j}$, i.e.,

$\mathbf{E}_{\mathrm{exc} i j}^{(i)}\left(\mathbf{r}_{i}\right)=\mathbf{E}_{\mathrm{sct} j}^{(j)}\left(\mathbf{r}_{j}\right)=\frac{\mathrm{e}^{\mathrm{j} \mathrm{k}_{1} \mathrm{r}_{\mathrm{j}}}}{r_{j}} \mathbf{E}_{\mathrm{sct} j}^{\infty}\left(\widehat{\mathbf{r}}_{j}\right) \approx \mathrm{e}^{\mathrm{j} k_{1} \widehat{\mathbf{R}}_{i j} \cdot \mathbf{r}_{i}} \mathbf{E}_{i j}$,

where

$\mathbf{E}_{i j}=\mathbf{E}_{\text {excij }}^{(i)}(0)=\frac{\mathrm{e}^{\mathrm{j} \mathrm{k}_{1} \mathrm{R}_{\mathrm{ij}}}}{R_{i j}} \mathbf{E}_{\mathrm{sct} j}^{\infty}\left(\widehat{\mathbf{R}}_{i j}\right)$.

Inserting Eq. (13) in Eq. (10) and using

$\mathbf{E}_{0}^{(i)}\left(\mathbf{r}_{i}\right)=\mathbf{E}_{0}\left(\mathbf{R}_{i}\right) \mathrm{e}^{\mathrm{j} \mathrm{k}_{1} \widehat{\hat{\mathbf{s}}} \cdot \mathbf{r}_{\mathrm{i}}}$

yields

$\mathbf{E}_{\text {exci }}^{(i)}\left(\mathbf{r}_{i}\right)=\mathbf{E}_{0}\left(\mathbf{R}_{i}\right) \mathrm{e}^{\mathrm{j} \mathrm{k}_{1} \widehat{\mathbf{s}} \cdot \mathbf{r}_{\mathrm{i}}}+\sum_{j \neq i} \mathbf{E}_{i j} \mathrm{e}^{\mathrm{j} k_{1} \widehat{\mathbf{R}}_{i j} \cdot \mathbf{r}_{i}}$, and we see that the field exciting particle $i, \mathbf{E}_{\text {exci }}^{(i)}\left(\mathbf{r}_{i}\right)$, a superposition of the incident plane electromagnetic wave of amplitude $\mathbf{E}_{0}\left(\mathbf{R}_{i}\right)$ propagating in the direction $\widehat{\mathbf{s}}$ and of local plane electromagnetic waves of amplitudes $\mathbf{E}_{i j}$ propagating in the directions $\widehat{\mathbf{R}}_{i j}$ and coming from all particles $j$ excepting $i$. The far-field pattern of the field scattered by particle $i$ is then

$\mathbf{E}_{\mathrm{scti} i}^{\infty}\left(\widehat{\mathbf{r}}_{i}\right)=\overline{\mathbf{A}}\left(\widehat{\mathbf{r}}_{i}, \widehat{\mathbf{s}}\right) \cdot \mathbf{E}_{0}\left(\mathbf{R}_{i}\right)+\sum_{j \neq i} \overline{\mathbf{A}}\left(\widehat{\mathbf{r}}_{i}, \widehat{\mathbf{R}}_{i j}\right) \cdot \mathbf{E}_{i j}$,

where $\overline{\mathbf{A}}$ is the far-field scattering dyadic [1-3]. With the notation

$\overline{\mathbf{U}}\left(\mathbf{r}_{i}, \widehat{\mathbf{s}}\right)=g_{0}\left(r_{i}\right) \overline{\mathbf{A}}\left(\widehat{\mathbf{r}}_{i}, \widehat{\mathbf{s}}\right)$,

where, excepting the normalization factor $4 \pi, g_{0}(r)=\exp \left(\mathrm{jk}_{1} \mathrm{r}\right) / \mathrm{r}$ is the scalar Green's function of wavenumber $k_{1}$, the total field is

$$
\begin{aligned}
\mathbf{E}(\mathbf{r}) & =\mathbf{E}_{0}(\mathbf{r})+\sum_{i} g_{0}\left(r_{i}\right) \mathbf{E}_{\mathrm{sct} i}^{\infty}\left(\widehat{\mathbf{r}}_{i}\right) \\
& =\mathbf{E}_{0}(\mathbf{r})+\sum_{i} \frac{\mathrm{e}^{\mathrm{j} \mathrm{k}_{1} \mathrm{r}_{\mathrm{i}}}}{r_{i}} \overline{\mathbf{A}}\left(\widehat{\mathbf{r}_{i}}, \widehat{\mathbf{s}}\right) \cdot \mathbf{E}_{0}\left(\mathbf{R}_{i}\right) \\
& +\sum_{i} \sum_{j \neq i} \frac{\mathrm{e}^{\mathrm{j} \mathrm{k}_{1} \mathrm{r}_{\mathrm{i}}}}{r_{i}} \overline{\mathbf{A}}\left(\widehat{\mathbf{r}}_{i}, \widehat{\mathbf{R}}_{i j}\right) \cdot \mathbf{E}_{i j} .
\end{aligned}
$$

Eq. (17) can be transformed into an implicit equation for the amplitudes $\mathbf{E}_{i j}$. Writing the far-field pattern representation (17) for the field scattered by particle $j$, choosing $\widehat{\mathbf{r}}_{j}=\widehat{\mathbf{R}}_{i j}$, multiplying the resulting equation by $g_{0}\left(R_{i j}\right)$, and using Eq. (14) give

$\mathbf{E}_{i j}=\overline{\mathbf{U}}\left(\mathbf{R}_{i j}, \widehat{\mathbf{s}}\right) \cdot \mathbf{E}_{0}\left(\mathbf{R}_{j}\right)+\sum_{k \neq j} \overline{\mathbf{U}}\left(\mathbf{R}_{i j}, \widehat{\mathbf{R}}_{j k}\right) \cdot \mathbf{E}_{j k}, \quad i \neq j$.

Eqs. (19) and (20) are the multiple scattering equations for sparse media, and are referred to as the far-field Foldy equations. The full derivation of the approximate far-field Foldy equations from the rigorous integral Foldy equations can be found in Refs. [2,3].

\section{The Twersky approximation}

Eq. (20) for the amplitudes of the exciting fields is solved by iteration. Inserting the iterated solution of this equation, otherwise known as the Neumann series, i.e.,

$$
\begin{aligned}
\mathbf{E}_{i j} & =\overline{\mathbf{U}}\left(\mathbf{R}_{i j}, \widehat{\mathbf{s}}\right) \cdot \mathbf{E}_{0}\left(\mathbf{R}_{j}\right) \\
& +\sum_{k \neq j} \overline{\mathbf{U}}\left(\mathbf{R}_{i j}, \widehat{\mathbf{R}}_{j k}\right) \cdot \overline{\mathbf{U}}\left(\mathbf{R}_{j k}, \widehat{\mathbf{s}}\right) \cdot \mathbf{E}_{0}\left(\mathbf{R}_{k}\right)+\cdots, i \neq j,
\end{aligned}
$$

into the equation for the total field (19), gives a series expansion for the total field. Each term in this series corresponds to a scattering path involving one or more particles. In a scattering path, a particle can appear once or more than once. The paths in which particles appear only once are called self-avoiding scattering paths, and in the Twersky approximation [8], only self-avoiding scattering paths are assumed to contribute to the total field. The result is an order-of-scattering expansion for the total field

$$
\begin{aligned}
\mathbf{E}(\mathbf{r}) & =\mathbf{E}_{0}(\mathbf{r})+\sum_{i} \overline{\mathbf{U}}\left(\mathbf{r}_{i}, \widehat{\mathbf{s}}\right) \cdot \mathbf{E}_{0}\left(\mathbf{R}_{i}\right) \\
& +\sum_{i} \sum_{j \neq i} \overline{\mathbf{U}}\left(\mathbf{r}_{i}, \widehat{\mathbf{R}}_{i j}\right) \cdot \overline{\mathbf{U}}\left(\mathbf{R}_{i j}, \widehat{\mathbf{s}}\right) \cdot \mathbf{E}_{0}\left(\mathbf{R}_{j}\right) \\
& +\sum_{i} \sum_{j \neq i} \sum_{k \neq i, j} \overline{\mathbf{U}}\left(\mathbf{r}_{i}, \widehat{\mathbf{R}}_{i j}\right) \cdot \overline{\mathbf{U}}\left(\mathbf{R}_{i j}, \widehat{\mathbf{R}}_{j k}\right) \\
& \cdot \overline{\mathbf{U}}\left(\mathbf{R}_{j k}, \widehat{\mathbf{s}}\right) \cdot \mathbf{E}_{0}\left(\mathbf{R}_{k}\right)+\cdots .
\end{aligned}
$$

The first term on the right-hand sides of Eq. (22) corresponds to the incident field, the second term corresponds to the sum of all waves scattered by one particle, the third term corresponds to the 
sum of all waves scattered by two particles, and so on. Note that since only self-avoiding scattering paths are considered, the terms corresponding to the case $k=i$ in the triple sum of Eq. (22) have been excluded. For large $N$, the Twersky approximation includes the majority of multiple-scattering paths. Indeed, for the $n$th order scattering term, the difference between the number of terms in the exact series $N(N-1)^{n-1}$ and the number of terms in the Twersky series $N ! /(N-n)$ ! goes to zero as $N \rightarrow \infty$. It should be pointed out that the order-of-scattering expansion (22) is a purely mathematical construct, and not a real physical phenomenon [2,3]. An important assumption which we make in the following is that the (farfield) Neumann series for the total field (22) converges uniformly with respect to $\mathbf{r}$. In this case, we can operate on the Neumann series (22), e.g., we can integrate the series term by term.

The order-of-scattering expansion for the total field can be represented diagramatically as

$\mathbf{E}(\mathbf{r})=\triangleleft-+\sum_{i}-\stackrel{i}{\circ} \triangleleft-+\sum_{i, j j \neq i}-\stackrel{i}{\circ}-\stackrel{j}{\circ} \triangleleft-+\cdots$.

Here, the symbol - represents the incident field $\mathbf{E}_{0}$, while the symbols $\circ$ and - mean multiplying a field by the dyadic $\overline{\mathbf{A}}$, and by the field propagator $g_{0}\left(r_{i}\right)$, respectively. Equivalently, the composed symbol $-\circ$ denotes multiplying a field by the dyadic $\overline{\mathbf{U}}$.

Let us give an interpretation of the Twersky approximation. For this purpose, we consider a configuration of $N$ particles $\Lambda_{N}=$ $\left\{\mathbf{R}_{1}, \ldots, \mathbf{R}_{N}\right\}$ and a configuration of $N-1$ particles $\Lambda_{N-1}^{i}$, in which particle $i$ is absent (removed from the group). Inserting the iterated solution for $\mathbf{E}_{i j}$ as given by Eq. (21) in Eq. (16) gives the expression for the field exciting particle $i$ at a point $\mathbf{r}_{i}$ near particle $i$ (Fig. 1(b)):

$$
\begin{aligned}
\mathbf{E}_{\mathrm{exci}}^{(i)}\left(\mathbf{r}_{i}\right) & =\mathbf{E}_{0}\left(\mathbf{R}_{i}\right) \mathrm{e}^{\mathrm{j} \mathrm{k}_{1} \widehat{\mathbf{s}} \cdot \mathbf{r}_{\mathrm{i}}} \\
& +\sum_{j \neq i} \mathrm{e}^{\mathrm{j} k_{1} \widehat{\mathbf{R}}_{i j} \cdot \mathbf{r}_{i}} \frac{\mathrm{e}^{\mathrm{j} \mathrm{k}_{1} \mathrm{R}_{\mathrm{ij}}}}{R_{i j}} \overline{\mathbf{A}}\left(\widehat{\mathbf{R}}_{i j}, \widehat{\mathbf{S}}\right) \cdot \mathbf{E}_{0}\left(\mathbf{R}_{j}\right) \\
& +\sum_{j \neq i} \sum_{k \neq i, j} \mathrm{e}^{\mathrm{j} k_{1} \widehat{\mathbf{R}}_{i j} \cdot \mathbf{r}_{i}} \frac{\mathrm{e}^{\mathrm{j} \mathrm{k}_{1} \mathrm{R}_{\mathrm{ij}}}}{\mathrm{e}_{i j}} \frac{\mathrm{e}^{\mathrm{j} \mathrm{k}_{1} \mathrm{R}_{\mathrm{jk}}}}{R_{j k}} \\
& \times \overline{\mathbf{A}}\left(\widehat{\mathbf{R}}_{i j}, \widehat{\mathbf{R}}_{j k}\right) \cdot \overline{\mathbf{A}}\left(\widehat{\mathbf{R}}_{j k}, \widehat{\mathbf{s}}\right) \cdot \mathbf{E}_{0}\left(\mathbf{R}_{k}\right)+\cdots
\end{aligned}
$$

Upon removing particle $i$ from the group, the total field at the same field point $\mathbf{r}_{i}$ (produced by the rest of $N-1$ particles) in the coordinate system centered at the origin of particle $i$ before the removal, is computed by applying the order-of-scattering expansion (22) to the configuration $\Lambda_{N-1}^{i}$ and at the field point $\mathbf{r}=\mathbf{R}_{i}+\mathbf{r}_{i}$. This field, denoted by $\mathbf{E}^{(i)}\left(\mathbf{r}_{i} \mid \Lambda_{N-1}^{i}\right)$, is given by

$$
\begin{aligned}
\mathbf{E}^{(i)}\left(\mathbf{r}_{i} \mid \Lambda_{N-1}^{i}\right) & =\mathbf{E}_{0}^{(i)}\left(\mathbf{r}_{i}\right)+\sum_{j \neq i} \frac{\mathrm{e}^{\mathrm{j} \mathrm{k}_{1} \mathrm{r}_{\mathrm{j}}}}{r_{j}} \overline{\mathbf{A}}\left(\widehat{\mathbf{r}}_{j}, \widehat{\mathbf{s}}\right) \cdot \mathbf{E}_{0}\left(\mathbf{R}_{j}\right) \\
& +\sum_{j \neq i} \sum_{k \neq i, j} \frac{\mathrm{e}^{\mathrm{j} \mathrm{k}_{1} \mathrm{r}_{\mathrm{j}}}}{r_{j}} \frac{\mathrm{e}^{\mathrm{j} \mathrm{k}_{1} \mathrm{R}_{\mathrm{jk}}}}{R_{j k}} \\
& \times \overline{\mathbf{A}}\left(\widehat{\mathbf{r}}_{j}, \widehat{\mathbf{R}}_{j k}\right) \cdot \overline{\mathbf{A}}\left(\widehat{\mathbf{R}}_{j k}, \widehat{\mathbf{s}}\right) \cdot \mathbf{E}_{0}\left(\mathbf{R}_{k}\right)+\cdots
\end{aligned}
$$

From Eqs. (24) and (25), we find by using the approximations $\widehat{\mathbf{r}}_{j} \approx$ $\widehat{\mathbf{R}}_{i j}$ and (12) as well as the incident field representation (15), that

$\mathbf{E}_{\text {exci }}^{(i)}\left(\mathbf{r}_{i}\right)=\mathbf{E}^{(i)}\left(\mathbf{r}_{i} \mid \Lambda_{N-1}^{i}\right)$.

In other words, in the framework of the Twersky approximation, the field exciting particle $i$ and calculated at a point $\mathbf{r}_{i}$ near particle $i$ is the total electric field that would exists at that point if the particle $i$ were removed from the group.

\section{Coherent field}

As we have mentioned in Section 3, averaging the total electromagnetic field over time is meaningless since it yields a zero

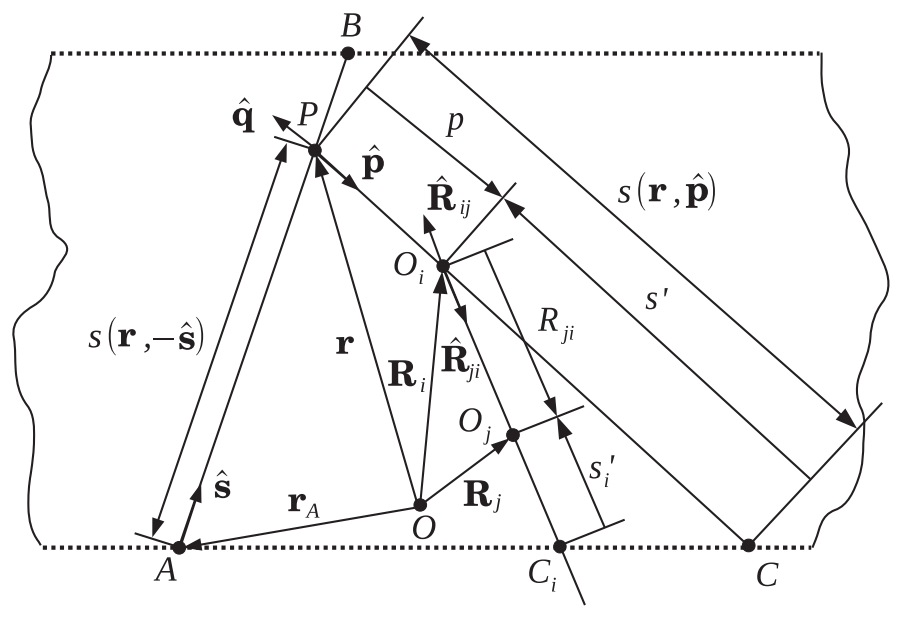

Fig. 2. Scattering geometry showing relevant quantities in computing integrals over particle positions.

result. However, it is sometimes instructive to consider the time average of a quantity obtained by artificially stripping the timeharmonic factor off the field. As usual, the temporal averaging is replaced by ensemble averaging based on the ergodicity assumption, and the resulting quantity is called the coherent field. It should always be recognized, however, that the coherent field is a purely mathematical, physically non-existent entity $[2,3,5]$.

The procedure of configuration averaging requires the computation of integrals over particle positions. To integrate over all positions of particle $i$ we use a local coordinate system with the origin at the observation point $P$, to integrate over all positions of particle $j$ we use a local coordinate system with the origin at particle $i$, and so on. In other words, we make the changes of variables

$\mathbf{R}_{i}=\mathbf{r}+\mathbf{p}, \quad \mathbf{R}_{j}=\mathbf{R}_{i}+\mathbf{R}_{j i}, \ldots$

The integration domain is $D$. As shown in Fig. 2, for direction $\widehat{\mathbf{p}}, p$ ranges from zero at the observation point $P$ to the corresponding value at the point $C$ (where the straight line with direction vector $\widehat{\mathbf{p}}$ crosses the lower plane boundary); for direction $\widehat{\mathbf{R}}_{j i}, R_{j i}$ ranges from zero at the origin of particle $i$ to the corresponding value at the point $C_{i}$ (where the straight line with direction vector $\widehat{\mathbf{R}}_{j i}$ crosses the lower plane boundary), etc.

Under the assumption that the positions of the particles are uncorrelated, the configuration average of the total field (22), or the coherent field $\mathbf{E}_{\mathrm{c}}(\mathbf{r})=\langle\mathbf{E}(\mathbf{r})\rangle$, is

$$
\begin{aligned}
\mathbf{E}_{\mathrm{c}}(\mathbf{r}) & =\mathbf{E}_{0}(\mathbf{r})+n_{0} \int_{D} \frac{\mathrm{e}^{\mathrm{j} \mathrm{k}_{1} \mathrm{r}_{\mathrm{i}}}}{r_{i}} \overline{\mathbf{A}}\left(\widehat{\mathbf{r}}_{i}, \widehat{\mathbf{s}}\right) \cdot \mathbf{E}_{0}\left(\mathbf{R}_{i}\right) \mathrm{d}^{3} \mathbf{R}_{i} \\
& +n_{0}^{2} \int_{D} \frac{\mathrm{e}^{\mathrm{j} \mathrm{k}_{1} \mathrm{r}_{\mathrm{i}}}}{\mathrm{e}_{i}} \frac{\mathrm{e}^{\mathrm{j} \mathrm{k}_{1} \mathrm{R}_{\mathrm{ij}}}}{R_{i j}} \\
& \times \overline{\mathbf{A}}\left(\widehat{\mathbf{r}}_{i}, \widehat{\mathbf{R}}_{i j}\right) \cdot \overline{\mathbf{A}}\left(\widehat{\mathbf{R}}_{i j}, \widehat{\mathbf{s}}\right) \cdot \mathbf{E}_{0}\left(\mathbf{R}_{j}\right) \mathrm{d}^{3} \mathbf{R}_{j} \mathrm{~d}^{3} \mathbf{R}_{i}+\cdots
\end{aligned}
$$

To compute the single-scattering term in Eq. (28), we make the change of variable (cf. Eq. (27)) $\mathbf{R}_{i}=\mathbf{r}+\mathbf{p}$, switch to spherical coordinates, i.e., $\mathrm{d}^{3} \mathbf{p}=p^{2} \mathrm{~d} p \mathrm{~d}^{2} \widehat{\mathbf{p}}$, and use the incident field representation

$\mathbf{E}_{0}\left(\mathbf{R}_{i}\right)=\mathrm{e}^{\mathrm{j} \mathrm{k}_{1} \widehat{\mathbf{s}} \cdot \mathbf{p}} \mathbf{E}_{0}(\mathbf{r})$

together with the asymptotic spherical waves representation for the plane wave $\exp \left(\mathrm{jk}_{1} \widehat{\mathbf{s}} \cdot \mathbf{p}\right)[9]$,

$\mathrm{e}^{\mathrm{j} k_{1} \widehat{\mathbf{s}} \mathbf{p}}=\frac{2 \pi}{\mathrm{j} k_{1} p}\left[\delta(\widehat{\mathbf{p}}-\widehat{\mathbf{s}}) \mathrm{e}^{\mathrm{j} k_{1} p}-\delta(\widehat{\mathbf{p}}+\widehat{\mathbf{s}}) \mathrm{e}^{-\mathrm{j} k_{1} p}\right], \quad k_{1} r \rightarrow \infty$,

where $\delta(\widehat{\mathbf{p}})$ is the solid-angle delta function. We thus obtain

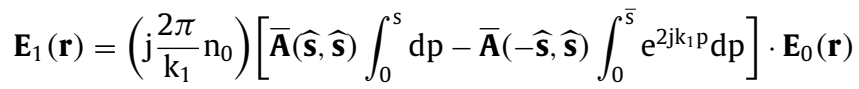




$$
=\left(\mathrm{j} \frac{2 \pi}{\mathrm{k}_{1}} \mathrm{n}_{0}\right)\left[\mathrm{s} \overline{\mathbf{A}}(\widehat{\mathbf{s}}, \widehat{\mathbf{s}})-\frac{\mathrm{e}^{2 \mathrm{j} \mathrm{k}_{1} \bar{s}}-1}{2 \mathrm{jk} \mathrm{k}_{1}} \overline{\mathbf{A}}(-\widehat{\mathbf{s}}, \widehat{\mathbf{s}})\right] \cdot \mathbf{E}_{0}(\mathbf{r}) .
$$

Here, $s=s(\mathbf{r},-\widehat{\mathbf{s}})=\widehat{\mathbf{s}} \cdot\left(\mathbf{r}-\mathbf{r}_{A}\right)$ and $\bar{s}=s(\mathbf{r}, \widehat{\mathbf{s}})=\widehat{\mathbf{s}} \cdot\left(\mathbf{r}_{B}-\mathbf{r}\right)$, where $\mathbf{r}_{A}$ and $\mathbf{r}_{B}$ are the points where the straight lines with the direction vectors $-\widehat{\mathbf{s}}$ and $\widehat{\mathbf{s}}$ going through the observation point cross the lower and the upper boundary of the layer, respectively. Because the medium is infinite in the horizontal directions, when switching to spherical coordinates, we exclude the direction characterized by the polar angle $\theta_{p}=\pi / 2$, where $\mathbf{p}=\left(p, \theta_{p}, \varphi_{p}\right)$; consequently, the single-scattering field is finite. By assuming that the particles are separated by distances much larger than the wavelength, i.e., $k_{1} s \gg 1$ (except for points in the immediate vicinity of the boundary), and taking account of

$\left|\frac{\mathrm{e}^{2 \mathrm{j} \mathrm{k}_{1} \overline{\mathrm{s}}}-1}{2 \mathrm{j}}\right|=\frac{1}{2}\left|\mathrm{e}^{2 \mathrm{j} \mathrm{k}_{1} \overline{\mathrm{s}}}-1\right| \leq \frac{1}{2}\left(\left|\mathrm{e}^{2 \mathrm{j} \mathrm{k}_{1} \overline{\mathrm{s}}}\right|+1\right)=1$,

we find

$s \gg \frac{1}{k_{1}} \geq\left|\frac{\mathrm{e}^{2 \mathrm{jk}_{1} \overline{\mathrm{s}}}-1}{2 \mathrm{jk}_{1}}\right|$.

Thus, the second term in the right-hand side of Eq. (31) is much smaller than the first term, and the result is

$\mathbf{E}_{1}(\mathbf{r})=\left(\mathrm{j} \frac{2 \pi}{\mathrm{k}_{1}} \mathrm{n}_{0} \mathrm{~s}\right) \overline{\mathbf{A}}(\widehat{\mathbf{s}}, \widehat{\mathbf{s}}) \cdot \mathbf{E}_{0}(\mathbf{r})$.

Similarly, for the double-scattering term in Eq. (28), we make the changes of variables $\mathbf{R}_{i}=\mathbf{r}+\mathbf{p}$ and $\mathbf{R}_{j}=\mathbf{R}_{i}+\mathbf{R}_{j i}$, and employ the spherical waves representations for the plane waves $\exp \left(j k_{1} \widehat{\mathbf{s}} \cdot \mathbf{p}\right)$ and $\exp \left(\mathrm{jk}_{1} \widehat{\mathbf{s}} \cdot \mathbf{R}_{\mathrm{ji}}\right)$; we get

$$
\begin{aligned}
\mathbf{E}_{2}(\mathbf{r}) & =n_{0}^{2} \int_{D} \frac{\mathrm{e}^{\mathrm{j} \mathrm{k}_{1} \mathrm{p}}}{p} \mathrm{e}^{\mathrm{j} \mathrm{k}_{1} \widehat{\mathbf{s}} \cdot \mathbf{p}}\left[\int_{D} \frac{\mathrm{e}^{\mathrm{j} \mathrm{k}_{1} \mathrm{R}_{\mathrm{ij}}}}{R_{i j}} \mathrm{e}^{\mathrm{j} \mathrm{k}_{1} \widehat{\widehat{s}} \mathbf{R}_{\mathrm{ji}}}\right. \\
& \left.\times \overline{\mathbf{A}}\left(-\widehat{\mathbf{p}},-\widehat{\mathbf{R}}_{j i}\right) \cdot \overline{\mathbf{A}}\left(-\widehat{\mathbf{R}}_{j i}, \widehat{\mathbf{s}}\right) \cdot \mathbf{E}_{0}(\mathbf{r}) \mathrm{d}^{3} \mathbf{R}_{j i}\right] \mathrm{d}^{3} \mathbf{p} \\
& =\left(\mathrm{j} \frac{2 \pi}{\mathrm{k}_{1}} \mathrm{n}_{0}\right)^{2} \overline{\mathbf{A}}(\widehat{\mathbf{s}}, \widehat{\mathbf{s}}) \cdot \overline{\mathbf{A}}(\widehat{\mathbf{s}}, \widehat{\mathbf{s}}) \cdot \mathbf{E}_{0}(\mathbf{r}) \int_{0}^{\mathrm{s}}\left(\int_{0}^{s-\mathrm{p}} \mathrm{dR} \mathrm{R}_{\mathrm{ji}}\right) \mathrm{dp} \\
& =\frac{1}{2}\left(\mathrm{j} \frac{2 \pi}{\mathrm{k}_{1}} \mathrm{n}_{0} \mathrm{~s}\right)^{2} \overline{\mathbf{A}}(\widehat{\mathbf{s}}, \widehat{\mathbf{s}}) \cdot \overline{\mathbf{A}}(\widehat{\mathbf{s}}, \widehat{\mathbf{s}}) \cdot \mathbf{E}_{0}(\mathbf{r}),
\end{aligned}
$$

Continuing this procedure, we find that Eq. (28) is the series expansion of a dyadic exponential function; the sum of the series is

$\mathbf{E}_{\mathrm{c}}(\mathbf{r})=\exp \left[\mathrm{j} \frac{2 \pi}{\mathrm{k}_{1}} \mathrm{n}_{0} \mathrm{~s}(\mathbf{r},-\widehat{\mathbf{s}}) \overline{\mathbf{A}}(\widehat{\mathbf{s}}, \widehat{\mathbf{s}})\right] \cdot \mathbf{E}_{0}(\mathbf{r})$.

From $\widehat{\mathbf{s}} \cdot \mathbf{E}_{0}(\mathbf{r})=0$ and $\widehat{\mathbf{s}} \cdot \overline{\mathbf{A}}(\widehat{\mathbf{s}}, \widehat{\mathbf{s}})=0$, we deduce that the coherent field is a transverse field, i.e., $\widehat{\mathbf{s}} \cdot \mathbf{E}_{\mathrm{c}}(\mathbf{r})=0$. Diagramatically, we have the representation

$$
\mathbf{E}_{\mathrm{c}}(\mathbf{r})=\triangleleft==\left\langle\triangleleft-+\sum_{i}-\stackrel{i}{\circ} \triangleleft-+\sum_{i, j j \neq i}-i \stackrel{\circ}{\circ}-\stackrel{\circ}{\bullet}-+\cdots\right\rangle,
$$

where the symbol $4=$ represents the coherent field.

Further transformations of Eq. (36) yield

$\mathbf{E}_{\mathrm{c}}(\mathbf{r})=\overline{\mathbf{t}}(\widehat{\mathbf{s}}, s(\mathbf{r},-\widehat{\mathbf{s}})) \cdot \mathbf{E}_{0}\left(\mathbf{r}_{A}\right)$,

where

$\overline{\mathbf{t}}(\widehat{\mathbf{s}}, s)=\exp [\mathrm{j} \overline{\mathbf{k}}(\widehat{\mathbf{s}}) s]$

with $s=s(\mathbf{r},-\widehat{\mathbf{s}})$ is the coherent transmission dyadic and

$\overline{\mathbf{k}}(\widehat{\mathbf{s}})=k_{1} \overline{\mathbf{I}}+\frac{2 \pi}{k_{1}} n_{0} \overline{\mathbf{A}}(\widehat{\mathbf{s}}, \widehat{\mathbf{s}})$,

with $\overline{\mathbf{I}}$ being the identity dyadic, is the dyadic propagation constant for direction $\widehat{\mathbf{s}}$. In matrix form, the dyadic propagation constant is

$\overline{\mathbf{k}}(\widehat{\mathbf{s}})=\sum_{\eta, \mu=s, \theta, \varphi}[\mathrm{K}(\widehat{\mathbf{s}})]_{\eta \mu} \widehat{\boldsymbol{\eta}}(\mathbf{s}) \otimes \widehat{\boldsymbol{\mu}}(\widehat{\mathbf{s}})$, where $\otimes$ is the dyadic product sign, and for $\eta=s, \theta, \varphi$, we have, respectively, $\widehat{\boldsymbol{\eta}}(\mathbf{s})=\widehat{\mathbf{s}}, \widehat{\boldsymbol{\theta}}(\mathbf{s}), \widehat{\boldsymbol{\varphi}}(\mathbf{s})$, and similarly for $\mu$. The $3 \times 3$ matrix K $(\widehat{\mathbf{s}})$ corresponding to the dyadic $\overline{\mathbf{k}}(\widehat{\mathbf{s}})$ is

$\mathrm{K}(\widehat{\mathbf{s}})=\left[\begin{array}{cc}k_{1} & 0 \\ 0 & \mathrm{k}(\widehat{\mathbf{s}})\end{array}\right]$,

and the $2 \times 2$ propagation constant matrix $\mathrm{k}(\boldsymbol{\mathrm { s }})$ is expressed in terms of the amplitude matrix $\mathrm{S}(\widehat{\mathbf{s}}, \widehat{\mathbf{s}})$ by the relation

$\mathrm{k}(\widehat{\mathbf{s}})=k_{1} \mathrm{I}_{2}+\frac{2 \pi}{k_{1}} n_{0} \mathrm{~S}(\widehat{\mathbf{s}}, \widehat{\mathbf{s}})$,

where $I_{2}$ is the $2 \times 2$ identity matrix. For the coherent transmission dyadic, we have a similar representation

$\overline{\mathbf{t}}(\widehat{\mathbf{s}}, s) \sum_{\eta, \mu=s, \theta, \varphi}[\mathrm{t}(\widehat{\mathbf{s}}, s)]_{\eta \mu} \widehat{\boldsymbol{\eta}}(\widehat{\mathbf{s}}) \otimes \widehat{\boldsymbol{\mu}}(\widehat{\mathbf{s}})$

with the coherent transmission matrix t being given by

$\mathrm{t}(\widehat{\mathbf{s}}, s)=\left[\begin{array}{cc}\exp \left(\mathrm{jk}_{1} \mathrm{~s}\right) & 0 \\ 0 & \exp [\mathrm{jk}(\widehat{\mathbf{s}}) \mathrm{s}]\end{array}\right]$.

Some properties of the coherent field are listed below $[2,3]$ :

1. Taking the derivative of Eq. (37) with respect to $s=s(\mathbf{r},-\widehat{\mathbf{s}})$, and using

$\frac{\mathrm{d} \overline{\mathbf{t}}(\widehat{\mathbf{s}}, s)}{\mathrm{d} s}=\mathrm{j} \overline{\mathbf{k}}(\mathbf{s}) \cdot \overline{\mathbf{t}}(\widehat{\mathbf{s}}, s)$,

gives

$\frac{\mathrm{d}_{\mathrm{c}}(\mathbf{r})}{\mathrm{d} s}=\mathrm{j} \overline{\mathbf{k}}(\widehat{\mathbf{s}}) \cdot \mathbf{E}_{\mathrm{c}}(\mathbf{r})$.

To find a matrix form representation of the above equation, we express the coherent field as

$\mathbf{E}_{\mathrm{c}}(\mathbf{r})=E_{\mathrm{c} \theta}(\mathbf{r}) \widehat{\boldsymbol{\theta}}(\widehat{\mathbf{s}})+E_{\mathrm{c} \varphi}(\mathbf{r}) \widehat{\boldsymbol{\varphi}}(\widehat{\mathbf{s}})$,

define the two-dimensional vector $\mathrm{E}_{\mathrm{c}}$ by

$\mathrm{E}_{\mathrm{c}}(\mathbf{r})=\left[\begin{array}{l}E_{\mathrm{c} \theta}(\mathbf{r}) \\ E_{\mathrm{c} \varphi}(\mathbf{r})\end{array}\right]$,

and obtain

$\frac{\mathrm{dE}_{\mathrm{c}}(\mathbf{r})}{\mathrm{d} s}=\mathrm{jk}(\widehat{\mathbf{s}}) \mathrm{E}_{\mathrm{c}}(\mathbf{r})$.

2. By direct calculation it can be shown that the coherent transmission dyadic satisfies the second-order ordinary differential equation

$\frac{\mathrm{d}^{2} \overline{\mathbf{t}}(\widehat{\mathbf{s}}, s)}{\mathrm{d} s^{2}}=-\overline{\mathbf{k}}^{2}(\widehat{\mathbf{s}}) \cdot \overline{\mathbf{t}}(\widehat{\mathbf{s}}, s)$.

As a result, the coherent field satisfies the dyadic wave equation

$\frac{\mathrm{d}^{2} \mathbf{E}_{\mathrm{c}}(\mathbf{r})}{\mathrm{d} s^{2}}+\overline{\mathbf{k}}^{2}(\widehat{\mathbf{s}}) \cdot \mathbf{E}_{\mathrm{c}}(\mathbf{r})=\mathbf{0}$.

3. Starting from the identity

$\frac{\mathrm{dE}_{\mathrm{c}}(\mathbf{r})}{\mathrm{d} s} \mathrm{E}_{\mathrm{c}}^{\dagger}(\mathbf{r})+\mathrm{E}_{\mathrm{c}}(\mathbf{r}) \frac{\mathrm{dE}_{\mathrm{c}}^{\dagger}(\mathbf{r})}{\mathrm{d} s}$

$=\frac{\mathrm{d}}{\mathrm{d} s}\left[\begin{array}{cc}\left|E_{\mathrm{c} \theta}(\mathbf{r})\right|^{2} & E_{\mathrm{c} \theta}(\mathbf{r}) E_{\mathrm{c} \varphi}^{\star}(\mathbf{r}) \\ E_{\mathrm{c} \varphi}(\mathbf{r}) E_{\mathrm{c} \theta}^{\star}(\mathbf{r}) & \left|E_{c \varphi}(\mathbf{r})\right|^{2}\end{array}\right]$,

where $\dagger$ stands for conjugate transpose, and using Eq. (49) together with Eqs. (40)-(42), we obtain

$\frac{\mathrm{d}_{\mathrm{c}}(\mathbf{r})}{\mathrm{d} s}=-n_{0} \mathrm{~K}_{\mathrm{J}}(\widehat{\mathbf{s}}) \mathrm{J}_{\mathrm{c}}(\mathbf{r})$, 
where

$\mathrm{J}_{\mathrm{c}}(\mathbf{r})=\frac{1}{2} \sqrt{\frac{\varepsilon_{1}}{\mu_{0}}}\left[\begin{array}{c}\left|E_{\mathrm{c} \theta}(\mathbf{r})\right|^{2} \\ E_{\mathrm{c} \theta}(\mathbf{r}) E_{c \varphi}^{\star}(\mathbf{r}) \\ E_{\mathrm{c} \varphi}(\mathbf{r}) E_{c \theta}^{\star}(\mathbf{r}) \\ \left|E_{\mathrm{c} \varphi}(\mathbf{r})\right|^{2}\end{array}\right]$

is the coherency column vector of the coherent field and $\mathrm{K}_{\mathrm{J}}$ is the coherency extinction matrix. Further, defining the Stokes column vector of the coherent field $I_{c}$ by

$\mathrm{I}_{\mathrm{c}}(\mathbf{r})=\mathrm{DJ}_{\mathrm{c}}(\mathbf{r})=\frac{1}{2} \sqrt{\frac{\varepsilon_{1}}{\mu_{0}}}\left[\begin{array}{c}\left|E_{c \theta}(\mathbf{r})\right|^{2}+\left|E_{c \varphi}(\mathbf{r})\right|^{2} \\ \left|E_{c \theta}(\mathbf{r})\right|^{2}-\left|E_{c \varphi}(\mathbf{r})\right|^{2} \\ -E_{\mathrm{c} \theta}(\mathbf{r}) E_{c \varphi}^{\star}(\mathbf{r})-E_{\mathrm{c} \varphi}(\mathbf{r}) E_{c \theta}^{\star}(\mathbf{r}) \\ \mathrm{j}\left[\mathrm{E}_{\mathrm{c} \varphi}(\mathbf{r}) \mathrm{E}_{\mathrm{c} \theta}^{\star}(\mathbf{r})-\mathrm{E}_{\mathrm{c} \theta}(\mathbf{r}) \mathrm{E}_{c \varphi}^{\star}(\mathbf{r})\right]\end{array}\right]$,

where $\mu_{0}$ is the magnetic permeability of a vacuum and the transformation matrix $\mathrm{D}$ is given by

$\mathrm{D}=\left[\begin{array}{cccc}1 & 0 & 0 & 1 \\ 1 & 0 & 0 & -1 \\ 0 & -1 & -1 & 0 \\ 0 & -\mathrm{j} & \mathrm{j} & 0\end{array}\right]$,

we find that $I_{c}(\mathbf{r})$ satisfies the differential equation

$\frac{\mathrm{dI}_{\mathrm{c}}(\mathbf{r})}{\mathrm{d} s}=-n_{0} \mathrm{~K}(\widehat{\mathbf{s}}) \mathrm{I}_{\mathrm{c}}(\mathbf{r})$,

with $\mathrm{K}(\widehat{\mathbf{s}})$ being the extinction matrix. Since, by assumption, the medium is homogeneous, $\mathrm{K}(\widehat{\mathbf{s}})$ does not change along the path $s$, and so the solution of the above equation is

$\mathrm{I}_{\mathrm{c}}(\mathbf{r})=\mathrm{e}^{-\mathrm{K}(\mathbf{s}) s(\mathbf{r},-\widehat{\mathbf{s}})} \mathrm{I}_{\mathrm{c}}\left(\mathbf{r}_{A}\right)$.

In Eq. (58), the matrix exponential $\mathrm{T}(\widehat{\mathbf{s}}, s)=\exp [-\mathrm{K}(\widehat{\mathbf{s}}) s(\mathbf{r},-\widehat{\mathbf{s}})]$ represents the coherent transmission Stokes matrix. Setting $\mathbf{r}=\mathbf{r}_{A}$ in Eq. (37) and accounting for $\overline{\mathbf{t}}(\widehat{\mathbf{s}}, 0)=\overline{\mathbf{I}}$, we obtain $\mathbf{E}_{\mathrm{c}}\left(\mathbf{r}_{A}\right)=\mathbf{E}_{0}\left(\mathbf{r}_{A}\right)$, and further

$\mathrm{I}_{\mathrm{c}}\left(\mathbf{r}_{A}\right)=\mathrm{I}_{0}(\mathbf{s})$.

Thus, the coherent Stokes column vector is the solution of the differential equation (57) with the initial condition (59).

\section{Inhomogeneous medium}

For an inhomogeneous medium, we follow Waterman and Truell [10] and define the density $n\left(\mathbf{R}_{i}\right)$ of particles at $\mathbf{R}_{i}$ by

$n\left(\mathbf{R}_{i}\right)=N p\left(\mathbf{R}_{i}\right)$.

In terms of densities, the joint probability $p\left(\mathbf{R}_{i}, \mathbf{R}_{j}\right)$ is

$p\left(\mathbf{R}_{i}, \mathbf{R}_{j}\right)=p\left(\mathbf{R}_{i}\right) p\left(\mathbf{R}_{j} \mid \mathbf{R}_{i}\right)=\frac{n\left(\mathbf{R}_{i}\right) n\left(\mathbf{R}_{j} \mid \mathbf{R}_{i}\right)}{N(N-1)}$

where we have taken into account that any one of the $N-1$ remaining particles has equal probability of residing in $\mathrm{d}^{3} \mathbf{R}_{j}$ if one particle resides in $\mathrm{d}^{3} \mathbf{R}_{i}$. The configuration average of the sum $\Sigma_{i} f(\mathbf{r}$, $\mathbf{R}_{i}$ ) is computed as

$\sum_{i}\left\langle f\left(\mathbf{r}, \mathbf{R}_{i}\right)\right\rangle=N \int f\left(\mathbf{r}, \mathbf{R}_{i}\right) p\left(\mathbf{R}_{i}\right) \mathrm{d}^{3} \mathbf{R}_{i}=\int f\left(\mathbf{r}, \mathbf{R}_{i}\right) n\left(\mathbf{R}_{i}\right) \mathrm{d}^{3} \mathbf{R}_{i}$,

and if the positions of the particles are independent, i.e., $n\left(\mathbf{R}_{j} \mid \mathbf{R}_{i}\right)=n\left(\mathbf{R}_{j}\right)$, the configuration average of the double sum $\Sigma_{i} \Sigma_{j \neq i} f\left(\mathbf{r}, \mathbf{R}_{i}, \mathbf{R}_{j}\right)$ is computed as

$\sum_{i} \sum_{j \neq i}\left\langle f\left(\mathbf{r}, \mathbf{R}_{i}, \mathbf{R}_{j}\right)\right\rangle$

$=N(N-1) \int f\left(\mathbf{r}, \mathbf{R}_{i}, \mathbf{R}_{j}\right) p\left(\mathbf{R}_{i}, \mathbf{R}_{j}\right) \mathrm{d}^{3} \mathbf{R}_{j} \mathrm{~d}^{3} \mathbf{R}_{i}$

$$
\approx \int f\left(\mathbf{r}, \mathbf{R}_{i}, \mathbf{R}_{j}\right) n\left(\mathbf{R}_{i}\right) n\left(\mathbf{R}_{j}\right) \mathrm{d}^{3} \mathbf{R}_{j} \mathrm{~d}^{3} \mathbf{R}_{i} .
$$

Applying these configuration average rules to the order-ofscattering expansion for the total field given by Eq. (22), we obtain (compare to Eq. (36))

$\mathbf{E}_{\mathrm{c}}(\mathbf{r})=\overline{\mathbf{T}}(\widehat{\mathbf{s}}, s) \cdot \mathbf{E}_{0}(\mathbf{r})$

where $s=s(\mathbf{r},-\widehat{\mathbf{s}})$, and with the notation of Fig. 2, the dyadic $\overline{\mathbf{T}}(\widehat{\mathbf{s}}, s)$ is

$$
\begin{aligned}
\overline{\mathbf{T}}(\widehat{\mathbf{s}}, s) & =\overline{\mathbf{I}}+\mathrm{j} \frac{2 \pi}{\mathrm{k}_{1}}\left[\int_{0}^{\mathrm{s}} \mathrm{n}\left(\mathrm{s}^{\prime}\right) \mathrm{ds} s^{\prime}\right] \overline{\mathbf{A}}(\widehat{\mathbf{s}}, \widehat{\mathbf{s}}) \\
& +\left(\mathrm{j} \frac{2 \pi}{\mathrm{k}_{1}}\right)^{2}\left\{\int_{0}^{s} \mathrm{n}\left(\mathrm{s}^{\prime}\right)\left[\int_{0}^{s^{\prime}} \mathrm{n}\left(\mathrm{s}_{\mathrm{i}}\right) \mathrm{d} s_{\mathrm{i}}\right] \mathrm{d} s^{\prime}\right\} \overline{\mathbf{A}}^{2}(\widehat{\mathbf{s}}, \widehat{\mathbf{s}})+\cdots .
\end{aligned}
$$

Assuming that the series (65) can be differentiated term by term, we find that $\overline{\mathbf{T}}$ satisfies the differential equation

$\frac{\mathrm{d} \overline{\mathbf{T}}(\widehat{\mathbf{s}}, s)}{\mathrm{d} s}=\mathrm{j} \frac{2 \pi}{\mathrm{k}_{1}} \mathrm{n}(\mathrm{s}) \overline{\mathbf{A}}(\widehat{\mathbf{s}}, \widehat{\mathbf{s}}) \cdot \overline{\mathbf{T}}(\widehat{\mathbf{s}}, s)$

with the initial condition $\overline{\mathbf{T}}(\widehat{\mathbf{s}}, 0)=\overline{\mathbf{I}}$. The solution to Eq. (66) is

$\overline{\mathbf{T}}(\widehat{\mathbf{s}}, s)=\exp \left\{\mathrm{j} \frac{2 \pi}{\mathrm{k}_{1}}\left[\int_{0}^{\mathrm{s}} \mathrm{n}\left(\mathrm{s}^{\prime}\right) \mathrm{d} \mathrm{s}^{\prime}\right] \overline{\mathbf{A}}(\widehat{\mathbf{s}}, \widehat{\mathbf{s}})\right\}$,

and we recover Eq. (37) with

$\overline{\mathbf{t}}(\widehat{\mathbf{s}}, s)=\exp \left[\mathrm{j} \int_{0}^{s} \overline{\mathbf{k}}\left(\widehat{\mathbf{s}}, \mathrm{s}^{\prime}\right) \mathrm{d} s^{\prime}\right]$

and

$\overline{\mathbf{k}}\left(\widehat{\mathbf{s}}, s^{\prime}\right)=k_{1} \overline{\mathbf{I}}+\frac{2 \pi}{k_{1}} n\left(s^{\prime}\right) \overline{\mathbf{A}}(\widehat{\mathbf{s}}, \widehat{\mathbf{s}})$.

\section{Multiple species of particles}

In this section, we extend the above results to the case where there are $P$ different species of particles. Each species can have a distinct size, shape, and permittivity, which in turn implies that each species $p=1, \ldots, P$ is characterized by the far-field scattering dyadic $\overline{\mathbf{A}}_{p}$. If $N_{p}$ is the number of particles of species $p$, we denote by $n_{p}=N_{p} / V$ the corresponding number concentration, and clearly, from $N=\sum_{p=1}^{P} N_{p}$, we see that $n_{0}=\sum_{p=1}^{P} n_{p}$.

For multiple species of particles, an order-of-scattering expansion for the total field reads as

$$
\begin{aligned}
\mathbf{E}(\mathbf{r}) & =\mathbf{E}_{0}(\mathbf{r})+\sum_{p=1}^{P} \sum_{i_{p}=1}^{N_{p}} \overline{\mathbf{U}}_{p}\left(\mathbf{r}_{i_{p}}, \widehat{\mathbf{s}}\right) \cdot \mathbf{E}_{0}\left(\mathbf{R}_{i_{p}}\right) \\
& +\sum_{p, q=1}^{P} \sum_{i_{p}=1}^{N_{p}} \sum_{j_{q}=1, j_{q} \neq i_{i} \delta_{p q}}^{N_{q}} \overline{\mathbf{U}}_{p}\left(\mathbf{r}_{i_{p}}, \widehat{\mathbf{R}}_{i_{p} j_{q}}\right) \\
& \cdot \overline{\mathbf{U}}_{q}\left(\mathbf{R}_{i_{p} j_{q}}, \widehat{\mathbf{s}}\right) \cdot \mathbf{E}_{0}\left(\mathbf{R}_{j_{q}}\right)+\cdots,
\end{aligned}
$$

with

$\overline{\mathbf{U}}_{p}\left(\mathbf{r}_{i_{p}}, \widehat{\mathbf{q}}\right)=g_{0}\left(r_{i_{p}}\right) \overline{\mathbf{A}}_{p}\left(\widehat{\mathbf{r}}_{i_{p}}, \widehat{\mathbf{q}}\right)$,

$\overline{\mathbf{U}}_{q}\left(\mathbf{R}_{i_{p} j_{q}}, \widehat{\mathbf{q}}\right)=g_{0}\left(R_{i_{p} j_{q}}\right) \overline{\mathbf{A}}_{q}\left(\widehat{\mathbf{R}}_{i_{p} j_{q}}, \widehat{\mathbf{q}}\right)$,

for any direction $\widehat{\mathbf{q}}$. Assuming that the positions of the particles of different species are independent and that these particles are uniformly distributed, we have

$p\left(\Lambda_{N_{1}}, \ldots, \Lambda_{N_{P}}\right)=\prod_{p=1}^{P} p\left(\Lambda_{N_{p}}\right)$,

with

$p\left(\Lambda_{N_{p}}\right)=\prod_{i_{p}=1}^{N_{p}} p\left(\mathbf{R}_{i_{p}}\right)$ and $p\left(\mathbf{R}_{i_{p}}\right)=1 / V$ 
where $\Lambda_{N_{p}}=\left\{\mathbf{R}_{i_{p}}\right\}_{i_{p}=1}^{N_{p}}$ is the spatial configuration of the particles of species $p$. Taking the configuration average of Eq. (70), we obtain

$$
\begin{aligned}
\mathbf{E}_{c}(\mathbf{r}) & =\mathbf{E}_{0}(\mathbf{r}) \\
& +\sum_{p=1}^{P} n_{p} \int_{D} \frac{\mathrm{e}^{\mathrm{j} \mathrm{k}_{1} \mathrm{r}_{\mathrm{p}}}}{r_{i_{p}}} \overline{\mathbf{A}}_{p}\left(\widehat{\mathbf{r}}_{i_{p}}, \widehat{\mathbf{s}}\right) \cdot \mathbf{E}_{0}\left(\mathbf{R}_{i_{p}}\right) \mathrm{d}^{3} \mathbf{R}_{i_{p}} \\
& +\sum_{p, q=1}^{P} n_{p} n_{q} \int_{D} \frac{\mathrm{e}^{\mathrm{j} \mathrm{k}_{1} \mathrm{r}_{\mathrm{i}_{\mathrm{p}}}}}{r_{i_{p}}} \frac{\mathrm{e}^{\mathrm{j} \mathrm{k}_{1} \mathrm{R}_{\mathrm{p} \mathrm{p} \mathrm{j}_{q}}}}{R_{i_{p} j_{q}}} \overline{\mathbf{A}}_{p}\left(\widehat{\mathbf{r}}_{i_{p}}, \widehat{\mathbf{R}}_{i_{p} j_{q}}\right) \\
& \cdot \overline{\mathbf{A}}_{q}\left(\widehat{\mathbf{R}}_{i_{p} j_{q}}, \widehat{\mathbf{s}}\right) \cdot \mathbf{E}_{0}\left(\mathbf{R}_{j_{q}}\right) \mathrm{d}^{3} \mathbf{R}_{j_{q}} \mathrm{~d}^{3} \mathbf{R}_{i_{p}}+\cdots
\end{aligned}
$$

The integrals in Eq. (75) are computed by using the same procedure as in Section 6; for example, we have

$\mathbf{E}_{1}(\mathbf{r})=\left(\mathrm{j} \frac{2 \pi}{\mathrm{k}_{1}} \mathrm{~s}\right)\left[\sum_{\mathrm{p}=1}^{\mathrm{P}} \mathrm{n}_{\mathrm{p}} \overline{\mathbf{A}}_{\mathrm{p}}(\widehat{\mathbf{s}}, \widehat{\mathbf{s}})\right] \cdot \mathbf{E}_{0}(\mathbf{r})$,

and

$$
\begin{aligned}
\mathbf{E}_{2}(\mathbf{r}) & =\frac{1}{2}\left(\mathrm{j} \frac{2 \pi}{\mathrm{k}_{1}} \mathrm{~s}\right)^{2}\left[\sum_{\mathrm{p}, \mathrm{q}=1}^{\mathrm{P}} \mathrm{n}_{\mathrm{p}} \mathrm{n}_{\mathrm{q}} \overline{\mathbf{A}}_{\mathrm{p}}(\widehat{\mathbf{s}}, \widehat{\mathbf{s}}) \cdot \overline{\mathbf{A}}_{\mathrm{q}}(\widehat{\mathbf{s}}, \widehat{\mathbf{s}})\right] \cdot \mathbf{E}_{0}(\mathbf{r}) \\
& =\frac{1}{2}\left(\mathrm{j} \frac{2 \pi}{\mathrm{k}_{1}} \mathrm{~s}\right)^{2}\left[\sum_{\mathrm{p}=1}^{\mathrm{P}} \mathrm{n}_{\mathrm{p}} \overline{\mathbf{A}}_{\mathrm{p}}(\widehat{\mathbf{s}}, \widehat{\mathbf{s}})\right]^{2} \cdot \mathbf{E}_{0}(\mathbf{r}) .
\end{aligned}
$$

Then, using the computation rule

$$
\sum_{p_{1}, \ldots, p_{k}=1}^{P} n_{p_{1}} \cdots n_{p_{k}} \overline{\mathbf{A}}_{p_{1}}(\widehat{\mathbf{s}}, \widehat{\mathbf{s}}) \cdot \ldots \cdot \overline{\mathbf{A}}_{p_{k}}(\widehat{\mathbf{s}}, \widehat{\mathbf{s}})=\left[\sum_{p=1}^{P} n_{p} \overline{\mathbf{A}}_{p}(\widehat{\mathbf{s}}, \widehat{\mathbf{s}})\right]^{k},
$$

where as usual, $\overline{\mathbf{X}}^{n}=\overline{\mathbf{X}}^{n-1} \cdot \overline{\mathbf{X}}$ for $n \geq 1$, we find that the coherent field is given by

$\mathbf{E}_{\mathrm{c}}(\mathbf{r})=\exp \left\{\mathrm{j} \frac{2 \pi}{\mathrm{k}_{1}} \mathrm{~s}(\mathbf{r},-\widehat{\mathbf{s}})\left[\sum_{\mathrm{p}=1}^{\mathrm{P}} \mathrm{n}_{\mathrm{p}} \overline{\mathbf{A}}_{\mathrm{p}}(\widehat{\mathbf{s}}, \widehat{\mathbf{s}})\right]\right\} \cdot \mathbf{E}_{0}(\mathbf{r})$.

Defining the species-averaged far-field scattering dyadic by

$\langle\overline{\mathbf{A}}(\widehat{\mathbf{s}}, \widehat{\mathbf{s}})\rangle_{\mathrm{s}}=\frac{1}{n_{0}} \sum_{p=1}^{P} n_{p} \overline{\mathbf{A}}_{p}(\widehat{\mathbf{s}}, \widehat{\mathbf{s}})=\frac{1}{N} \sum_{p=1}^{P} N_{p} \overline{\mathbf{A}}_{p}(\widehat{\mathbf{s}}, \widehat{\mathbf{s}})$,

we express Eq. (79) as

$\mathbf{E}_{\mathrm{c}}(\mathbf{r})=\exp \left[\mathrm{j} \frac{2 \pi}{\mathrm{k}_{1}} \mathrm{n}_{0} \mathrm{~s}(\mathbf{r},-\widehat{\mathbf{s}})\langle\overline{\mathbf{A}}(\widehat{\mathbf{s}}, \widehat{\mathbf{s}})\rangle_{\mathrm{s}}\right] \cdot \mathbf{E}_{0}(\mathbf{r})$.

Thus, the representation for the coherent field given by Eq. (36) remains valid with the species-averaged dyadic $\langle\overline{\mathbf{A}}(\widehat{\mathbf{s}}, \widehat{\mathbf{s}})\rangle_{\mathrm{s}}$ in place of $\overline{\mathbf{A}}(\widehat{\mathbf{s}}, \widehat{\mathbf{s}})$. In particular, for spherical particles with a continuous size distribution characterized by the probability density function $p(a)$, the species- or the size-averaged far-field scattering dyadic is (cf. Eq. (80))

$\langle\overline{\mathbf{A}}(\widehat{\mathbf{s}}, \widehat{\mathbf{s}})\rangle_{\mathbf{s}}=\frac{1}{N} \int_{0}^{\infty} \overline{\mathbf{A}}_{a}(\widehat{\mathbf{s}}, \widehat{\mathbf{s}}) \mathrm{d} N(a)=\int_{0}^{\infty} p(a) \overline{\mathbf{A}}_{a}(\widehat{\mathbf{s}}, \widehat{\mathbf{s}}) \mathrm{d} a$,

where $a$ is the particle radius, $\left.\overline{\mathbf{A}}_{a} \widehat{\mathbf{s}}, \widehat{\mathbf{s}}\right)$ is the far-field scattering dyadic of a spherical particle of radius $a$, and $\mathrm{d} N(a)=N p(a) \mathrm{d} a$ is the number of particles with the radius in the interval $[a, a+\mathrm{d} a]$.

\section{Continuous extension of the far-field representation}

In the above derivation we employed the far-field approximation which assumes that each particle is located in the far-field regions of all the other particles, and that the observation point is also located in the far-field region of any particle. Although the scattered field representations are valid in the far-field regions, when computing the configuration average and integrating over the particle positions we allowed the particles to come into the near-field regions of each other. This approach tacitly assumes that the far-field representation of the scattered field is "extended by continuity" to the near-field region. To explain the meaning of this process, we use the hole-correction approximation. In this case, the probability density function $p\left(\mathbf{R}_{i} ; \mathbf{r}\right)$ is constructed as

$$
\begin{aligned}
p\left(\mathbf{R}_{i} ; \mathbf{r}\right) & =\frac{1}{V-V_{R}} \Theta_{R}\left(\mathbf{R}_{i} ; \mathbf{r}\right), \\
\Theta_{R}\left(\mathbf{R}_{i} ; \mathbf{r}\right) & := \begin{cases}1, & \mathbf{R}_{i} \in D-D_{R}(\mathbf{r}), \\
0, & \text { rest }\end{cases}
\end{aligned}
$$

where $D$ is the domain occupied by the particles, $D_{R}(\mathbf{r})$ is a ball of radius $R$ around $\mathbf{r}$ with the property that the far-field approximation of the scattered field is valid in the exterior of $D_{R}(\mathbf{r})$, and $V_{R}=(4 / 3) \pi R^{3}$. The joint probability $p\left(\mathbf{R}_{i}, \mathbf{R}_{j} ; \mathbf{r}\right)$ is constructed in a similar manner; we have

$$
\begin{aligned}
p\left(\mathbf{R}_{i}, \mathbf{R}_{j} ; \mathbf{r}\right) & =p\left(\mathbf{R}_{i} ; \mathbf{r}\right) p\left(\mathbf{R}_{j} \mid \mathbf{R}_{i} ; \mathbf{r}\right), \\
p\left(\mathbf{R}_{j} \mid \mathbf{R}_{i} ; \mathbf{r}\right) & =\frac{1}{V-2 V_{R}} \Theta_{R}\left(\mathbf{R}_{j} \mid \mathbf{R}_{i} ; \mathbf{r}\right), \\
\Theta_{R}\left(\mathbf{R}_{j} \mid \mathbf{R}_{i} ; \mathbf{r}\right) & := \begin{cases}1, & \mathbf{R}_{j} \in D-\left(D_{R}(\mathbf{r}) \cup D_{R}\left(\mathbf{R}_{i}\right)\right), \\
0, & \text { rest }\end{cases}
\end{aligned}
$$

showing that (cf. Eq. (3))

$$
\begin{aligned}
1 & =\int_{D} p\left(\mathbf{R}_{i}, \mathbf{R}_{j} ; \mathbf{r}\right) \mathrm{d}^{3} \mathbf{R}_{i} \mathrm{~d}^{3} \mathbf{R}_{j} \\
& =\int_{D} p\left(\mathbf{R}_{i} ; \mathbf{r}\right)\left[\int_{D} p\left(\mathbf{R}_{j} \mid \mathbf{R}_{i} ; \mathbf{r}\right) \mathrm{d}^{3} \mathbf{R}_{j}\right] \mathrm{d}^{3} \mathbf{R}_{i} .
\end{aligned}
$$

Thus, the hole-correction approximation takes into account that each particle is situated outside the far-field spheres of all the other particles, and further assumes the uniform distribution outside the far-field sphere around the observation point. The configuration average of a function $f\left(\mathbf{r}, \mathbf{R}_{i}\right)$ is computed as

$\left\langle f\left(\mathbf{r}, \mathbf{R}_{i}\right)\right\rangle=\int f\left(\mathbf{r}, \mathbf{R}_{i}\right) p\left(\mathbf{R}_{i} ; \mathbf{r}\right) \mathrm{d}^{3} \mathbf{R}_{i}$,

while for a function $f\left(\mathbf{r}, \mathbf{R}_{i}, \mathbf{R}_{j}\right)$, we have

$\left\langle f\left(\mathbf{r}, \mathbf{R}_{i}, \mathbf{R}_{j}\right)\right\rangle=\int f\left(\mathbf{r}, \mathbf{R}_{i}, \mathbf{R}_{j}\right) p\left(\mathbf{R}_{i}, \mathbf{R}_{j} ; \mathbf{r}\right) \mathrm{d}^{3} \mathbf{R}_{j} \mathrm{~d}^{3} \mathbf{R}_{i}$.

Taking the configuration average of the total field in Eq. (22), and accounting of Eqs. (85) and (86), we obtain (compare to Eq. (28)),

$$
\begin{aligned}
\mathbf{E}_{\mathrm{c}}(\mathbf{r}, R) & =\mathbf{E}_{0}(\mathbf{r})+N \int_{D} \frac{\mathrm{e}^{\mathrm{j} \mathrm{k}_{1} \mathrm{r}_{\mathrm{i}}}}{r_{i}} \overline{\mathbf{A}}\left(\widehat{\mathbf{r}_{i}}, \widehat{\mathbf{s}}\right) \cdot \mathbf{E}_{0}\left(\mathbf{R}_{i}\right) p\left(\mathbf{R}_{i} ; \mathbf{r}\right) \mathrm{d}^{3} \mathbf{R}_{i} \\
& +N(N-1) \int_{D} \frac{\mathrm{e}^{\mathrm{j} \mathrm{k}_{1} \mathrm{r}_{\mathrm{i}}}}{r_{i}} \frac{\mathrm{e}^{\mathrm{j} \mathrm{k}_{1} \mathrm{R}_{\mathrm{ij}}}}{R_{i j}} \overline{\mathbf{A}}\left(\widehat{\mathbf{r}}_{i}, \widehat{\mathbf{R}}_{i j}\right) \cdot \overline{\mathbf{A}}\left(\widehat{\mathbf{R}}_{i j}, \widehat{\mathbf{s}}\right) \\
& \cdot \mathbf{E}_{0}\left(\mathbf{R}_{j}\right) p\left(\mathbf{R}_{i} ; \mathbf{r}\right) p\left(\mathbf{R}_{j} \mid \mathbf{R}_{i} ; \mathbf{r}\right) \mathrm{d}^{3} \mathbf{R}_{j} \mathrm{~d}^{3} \mathbf{R}_{i}+\cdots .
\end{aligned}
$$

The technique for computing the integrals in Eq. (87) also relies on the asymptotic representation of a plane wave in spherical waves by means of solid-angle delta functions. For example, and referring to Fig. 3, the double-scattering term in Eq. (87) computes as

$$
\begin{aligned}
\mathbf{E}_{2}(\mathbf{r}, R) & =N(N-1) \int_{D} \frac{\mathrm{e}^{\mathrm{j} \mathrm{k}_{1} \mathrm{r}_{\mathrm{i}}}}{r_{i}} \frac{\mathrm{e}^{\mathrm{j} \mathrm{k}_{1} \mathrm{R}_{\mathrm{ij}}}}{R_{i j}} \overline{\mathbf{A}}\left(\widehat{\mathbf{r}}_{i}, \widehat{\mathbf{R}}_{i j}\right) \cdot \overline{\mathbf{A}}\left(\widehat{\mathbf{R}}_{i j}, \widehat{\mathbf{s}}\right) \\
& \cdot \mathbf{E}_{0}\left(\mathbf{R}_{j}\right) p\left(\mathbf{R}_{i} ; \mathbf{r}\right) p\left(\mathbf{R}_{j} \mid \mathbf{R}_{i} ; \mathbf{r}\right) \mathrm{d}^{3} \mathbf{R}_{j} \mathrm{~d}^{3} \mathbf{R}_{i} \\
& =\frac{N(N-1)}{\left(V-V_{R}\right)\left(V-2 V_{R}\right)}\left(\mathrm{j} \frac{2 \pi}{\mathrm{k}_{1}}\right)^{2} \overline{\mathbf{A}}(\widehat{\mathbf{s}}, \widehat{\mathbf{s}}) \cdot \overline{\mathbf{A}}(\widehat{\mathbf{s}}, \widehat{\mathbf{s}}) \cdot \mathbf{E}_{0}(\mathbf{r}) \\
& \times \int_{0}^{s} h(p \mid R, s-R)\left[\int_{0}^{s} h\left(R_{j i} \mid R, s-p-2 R\right) \mathrm{d} R_{j i}\right] \mathrm{d} p,
\end{aligned}
$$




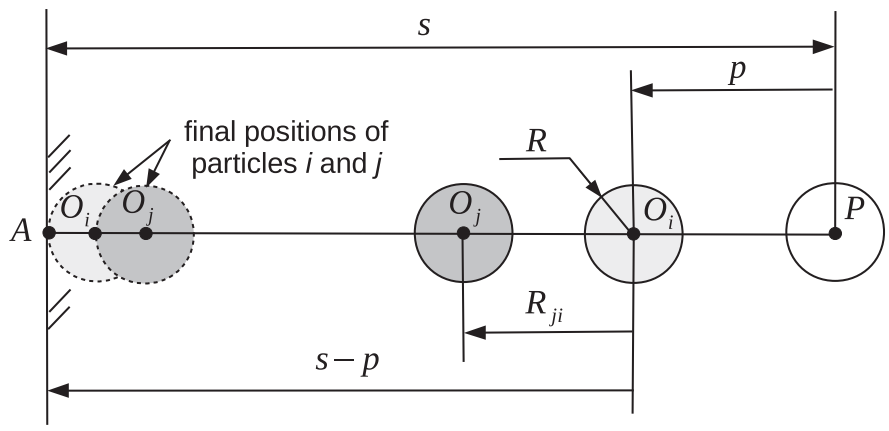

Fig. 3. Geometry showing the positions of particles $i$ and $j$ for computing the integral (88). The boundary is situated in the far-field region of particle $i$.

where $s=s(\mathbf{r},-\widehat{\mathbf{s}})$, and the function $h(x \mid a, b)$ is defined by

$h(x \mid a, b)=\left\{\begin{array}{l}1, \text { if } a \leq b \text { and } a \leq x \leq b \\ 0, \text { rest }\end{array}\right.$

It is not hard to check that in the limit $R \rightarrow 0$, we have

$\lim _{R \rightarrow 0} \int_{0}^{s} h(p \mid R, s-R)\left[\int_{0}^{s} h\left(R_{j i} \mid R, s-p-2 R\right) \mathrm{d} R_{j i}\right] \mathrm{d} p$

$=\int_{0}^{s} h(p \mid 0, s)\left[\int_{0}^{s} h\left(R_{j i} \mid 0, s-p\right) \mathrm{d} R_{j i}\right] \mathrm{d} p$

$=\int_{0}^{s}\left(\int_{0}^{s-p} \mathrm{~d} R_{j i}\right) \mathrm{d} p$

so that by approximating

$\lim _{R \rightarrow 0} \frac{N(N-1)}{\left(V-V_{R}\right)\left(V-2 V_{R}\right)}=n_{0}^{2}$,

we find that $\lim _{R \rightarrow 0} \mathbf{E}_{2}(\mathbf{r}, R)=\mathbf{E}_{2}(\mathbf{r})$, where $\mathbf{E}_{2}(\mathbf{r})$ is given by Eq. (35). Similar estimates hold for the rest of the terms in the series (87). Assuming now that the series (87) converges uniformly with respect to $R$, we get $\lim _{R \rightarrow 0} \mathbf{E}_{\mathrm{c}}(\mathbf{r}, R)=\mathbf{E}_{\mathrm{c}}(\mathbf{r})$; hence $\mathbf{E}_{\mathrm{c}}(\mathbf{r})$ is the limit of $\mathbf{E}_{\mathrm{c}}(\mathbf{r}, R)$ at the point $R=0$.

\section{The Foldy approximation}

We have seen that according to the Twersky approximation, the field exciting particle $i$ at a point $\mathbf{r}_{i}$ near particle $i$ is the total electric field that would exists at that point if the particle $i$ were removed from the group, i.e., (cf. Eq. (26))

$\mathbf{E}_{\text {exci }}^{(i)}\left(\mathbf{r}_{i}\right)=\mathbf{E}^{(i)}\left(\mathbf{r}_{i} \mid \Lambda_{N-1}^{i}\right)$.

Taking the configuration average of Eq. (90) with the position of particle $i$ held fixed, and using Eq. (5) yields

$\left\langle\mathbf{E}_{\text {exci }}^{(i)}\left(\mathbf{r}_{i}\right)\right\rangle_{i}=\mathbf{E}_{\mathrm{c}}^{(i)}\left(\mathbf{r}_{i} \mid \Lambda_{N-1}^{i}\right)$.

Thus, the average field exciting particle $i$ at a point $\mathbf{r}_{i}$ near particle $i$ is the coherent field that would exists at that point if the particle $i$ were removed from the group.

The Foldy approximation [11] states that the coherent field at a point $\mathbf{r}_{i}$ near particle $i$ is the conditional average of the field exciting particle $i$ at that point,

$\mathbf{E}_{\mathrm{c}}^{(i)}\left(\mathbf{r}_{i}\right)=\left\langle\mathbf{E}_{\text {exci }}^{(i)}\left(\mathbf{r}_{i}\right)\right\rangle_{i}$.

There are some interesting results which follow from the derivation of the coherent field under the Twersky approximation.

Corollary 1: The Twersky approximation implies the Foldy approximation. To show this, we take the configuration average of $\mathbf{E}_{\text {exci }}^{(i)}\left(\mathbf{r}_{i}\right)$ (given by Eq. (24)) with the position of particle $i$ held fixed, and employ exactly the same technique as that used when computing the coherent field; we obtain

$\left\langle\mathbf{E}_{\text {exci }}^{(i)}\left(\mathbf{r}_{i}\right)\right\rangle_{i}=\mathrm{e}^{\mathrm{j} \mathrm{k}_{1} \widehat{\widehat{s}} \cdot \mathbf{r}_{\mathrm{i}}} \exp \left[\mathrm{j} \frac{2 \pi}{\mathrm{k}_{1}} \mathrm{n}_{0} \mathrm{~s}\left(\mathbf{R}_{\mathrm{i}},-\widehat{\mathbf{s}}\right) \overline{\mathbf{A}}(\widehat{\mathbf{s}}, \widehat{\mathbf{s}})\right] \cdot \mathbf{E}_{0}\left(\mathbf{R}_{\mathrm{i}}\right)$.
On the other hand, setting $\mathbf{r}=\mathbf{R}_{i}+\mathbf{r}_{i}$ in Eq. (36) and using $\mathbf{E}_{0}\left(\mathbf{r}_{i}\right)=$ $\exp \left(\mathrm{jk}_{1} \widehat{\mathbf{s}} \cdot \mathbf{r}_{\mathrm{i}}\right) \mathbf{E}_{0}\left(\mathbf{R}_{\mathrm{i}}\right)$ gives

$\mathbf{E}_{\mathrm{c}}(\mathbf{r})=\mathbf{E}_{\mathrm{c}}^{(i)}\left(\mathbf{r}_{i}\right)=\mathrm{e}^{\mathrm{j} \mathrm{k}_{1} \widehat{\widehat{s}} \cdot \mathbf{r}_{\mathrm{i}}} \exp \left[\mathrm{j} \frac{2 \pi}{\mathrm{k}_{1}} \mathrm{n}_{0} \mathrm{~s}(\mathbf{r},-\widehat{\mathbf{s}}) \overline{\mathbf{A}}(\widehat{\mathbf{s}}, \widehat{\mathbf{s}})\right] \cdot \mathbf{E}_{0}\left(\mathbf{R}_{\mathrm{i}}\right)$.

If the point $\mathbf{r}_{i}$ is near particle $i$, and the boundary is in the far-field region of particle $i$, we approximate

$s\left(\mathbf{R}_{i},-\widehat{\mathbf{s}}\right) \approx s(\mathbf{r},-\widehat{\mathbf{s}})$.

Hence, from Eqs. (93) and (94), the Foldy approximation (92) readily follows. As a consequence, we note that by owing to Eqs. (91) and (92), we have

$\mathbf{E}_{\mathrm{c}}^{(i)}\left(\mathbf{r}_{i}\right)=\mathbf{E}_{\mathrm{c}}^{(i)}\left(\mathbf{r}_{i} \mid \Lambda_{N-1}^{i}\right)$,

that is, the coherent field at a point $\mathbf{r}_{i}$ near particle $i$ is the coherent field that would exist at that point if the particle $i$ were removed from the group.

Corollary 2: The coherent field near a particle can be analytically approximated by a plane electromagnetic wave of wavenumber $k_{1}$ and propagation direction $\widehat{\mathbf{s}}$. To prove this assertion, we set $\mathbf{r}=\mathbf{R}_{i}\left(\mathbf{r}_{i}=\right.$ 0 ) in Eq. (94) and use Eq. (93) to obtain

$$
\begin{aligned}
\mathbf{E}_{\mathrm{c}}\left(\mathbf{R}_{i}\right) & =\exp \left[\mathrm{j} \frac{2 \pi}{\mathrm{k}_{1}} \mathrm{n}_{0} \mathrm{~s}\left(\mathbf{R}_{\mathrm{i}},-\widehat{\mathbf{s}}\right) \overline{\mathbf{A}}(\widehat{\mathbf{s}}, \widehat{\mathbf{s}})\right] \cdot \mathbf{E}_{0}\left(\mathbf{R}_{\mathrm{i}}\right) \\
& =\mathrm{e}^{-\mathrm{j} \mathrm{k}_{1} \widehat{\mathbf{s}} \cdot \mathbf{r}_{\mathrm{i}}}\left\langle\mathbf{E}_{\text {exci }}^{(i)}\left(\mathbf{r}_{i}\right)\right\rangle_{i} ;
\end{aligned}
$$

thus,

$\left\langle\mathbf{E}_{\mathrm{exci}}^{(i)}\left(\mathbf{r}_{i}\right)\right\rangle_{i}=\mathrm{e}^{\mathrm{j} \mathrm{k}_{1} \widehat{\widehat{s}} \cdot \mathbf{r}_{\mathrm{i}}} \mathbf{E}_{\mathrm{c}}\left(\mathbf{R}_{i}\right)$.

From Eqs. (92) and (98), we then get

$\mathbf{E}_{\mathrm{c}}^{(i)}\left(\mathbf{r}_{i}\right)=\mathrm{e}^{\mathrm{j} \mathrm{k}_{1} \widehat{\widehat{s}} \cdot \mathbf{r}_{\mathrm{i}}} \mathbf{E}_{\mathrm{c}}\left(\mathbf{R}_{i}\right)$,

and the assertion is proved.

Corollary 3: The coherent field solves an integral equation, the socalled Foldy integral equation. To derive this equation, we consider the expression of the far-field pattern $\mathbf{E}_{\text {scti }}^{\infty}\left(\widehat{\mathbf{r}}_{i}\right)$ as given by Eq. (17). Inserting the iterated solution for $\mathbf{E}_{i j}$ given by Eq. (21) in Eq. (17), we find

$$
\begin{aligned}
\mathbf{E}_{\mathrm{scti} i}^{\infty}\left(\widehat{\mathbf{r}}_{i}\right) & =\overline{\mathbf{A}}\left(\widehat{\mathbf{r}}_{i}, \widehat{\mathbf{s}}\right) \cdot \mathbf{E}_{0}\left(\mathbf{R}_{i}\right) \\
& +\sum_{j \neq i} \frac{\mathrm{e}^{\mathrm{j} \mathrm{k}_{1} \mathrm{R}_{\mathrm{ij}}}}{R_{i j}} \overline{\mathbf{A}}\left(\widehat{\mathbf{r}}_{i}, \widehat{\mathbf{R}}_{i j}\right) \cdot \overline{\mathbf{A}}\left(\widehat{\mathbf{R}}_{i j}, \widehat{\mathbf{s}}\right) \cdot \mathbf{E}_{0}\left(\mathbf{R}_{j}\right) \\
& +\sum_{j \neq i} \sum_{k \neq i, j} \frac{\mathrm{e}^{\mathrm{j} \mathrm{k}_{1} \mathrm{R}_{\mathrm{ij}}}}{R_{i j}} \frac{\mathrm{e}^{\mathrm{j} \mathrm{j}_{1} \mathrm{R}_{\mathrm{jk}}}}{R_{j k}} \overline{\mathbf{A}}\left(\widehat{\mathbf{r}}_{i}, \widehat{\mathbf{R}}_{i j}\right) \\
& \cdot \overline{\mathbf{A}}\left(\widehat{\mathbf{R}}_{i j}, \widehat{\mathbf{R}}_{j k}\right) \cdot \overline{\mathbf{A}}\left(\widehat{\mathbf{R}}_{j k}, \widehat{\mathbf{s}}\right) \cdot \mathbf{E}_{0}\left(\mathbf{R}_{k}\right)+\cdots
\end{aligned}
$$

Taking the conditional average of Eq. (100) and applying the same technique as that used when computing the coherent field, we obtain

$\left\langle\mathbf{E}_{\mathrm{scti} i}^{\infty}\left(\widehat{\mathbf{r}}_{i}\right)\right\rangle_{i}=\overline{\mathbf{A}}\left(\widehat{\mathbf{r}}_{i}, \widehat{\mathbf{s}}\right) \cdot \exp \left[\mathrm{j} \frac{2 \pi}{\mathrm{k}_{1}} \mathrm{n}_{0} \mathrm{~s}\left(\mathbf{R}_{\mathrm{i}}\right) \overline{\mathbf{A}}(\widehat{\mathbf{s}}, \widehat{\mathbf{s}})\right] \cdot \mathbf{E}_{0}\left(\mathbf{R}_{\mathrm{i}}\right)$.

From Eqs. (93) and (101), we see that

$\left\langle\mathbf{E}_{\mathrm{sct} i}^{\infty}\left(\widehat{\mathbf{r}}_{i}\right)\right\rangle_{i}=\mathrm{e}^{-\mathrm{jk} \mathbf{k}_{1} \cdot \mathbf{r}_{\mathrm{i}}} \overline{\mathbf{A}}\left(\widehat{\mathbf{r}}_{i}, \widehat{\mathbf{s}}\right) \cdot\left\langle\mathbf{E}_{\mathrm{exc} i}^{(i)}\left(\mathbf{r}_{i}\right)\right\rangle_{i}$.

The configuration average of the first equation in Eq. (19) is

$\mathbf{E}_{\mathrm{c}}(\mathbf{r})=\mathbf{E}_{0}(\mathbf{r})+n_{0} \int_{D} g_{0}\left(r_{i}\right)\left\langle\mathbf{E}_{\mathrm{sct} i}^{\infty}\left(\widehat{\mathbf{r}}_{i}\right)\right\rangle_{i} \mathrm{~d}^{3} \mathbf{R}_{i}$,

so that using Eqs. (98) and (102), we find

$\mathbf{E}_{\mathrm{c}}(\mathbf{r})=\mathbf{E}_{0}(\mathbf{r})+n_{0} \int_{D} g_{0}\left(r_{i}\right) \overline{\mathbf{A}}\left(\widehat{\mathbf{r}}_{i}, \widehat{\mathbf{s}}\right) \cdot \mathbf{E}_{\mathrm{c}}\left(\mathbf{R}_{i}\right) \mathrm{d}^{3} \mathbf{R}_{i}$.

Eq. (104) is the Foldy integral equation for the coherent field [12]. Basically, we showed that if the coherent field is given by Eq. (36), 
that is, derived under the Twersky approximation, then it satisfies the Foldy integral equation (104). The converse result, namely that the solution of Eq. (104) is Eq. (36), will be proved in a forthcoming paper.

\section{Second-order moment in the electromagnetic field}

In this section we are concerned with the computation of the configuration average of energetic quantities, which are quadratic in the field amplitudes. The configuration average of an energetic quantity must be computed explicitly, because the process of averaging cannot be expect to commute with the nonlinear operation of squaring the absolute value of a field quantity. From the perspective of quantifying the reading of a (polarizationsensitive) well-collimated radiometer [3], the most appropriate quantity to average over the ensemble is the dyadic correlation function. However, we will simplify the discussion by considering the configuration-averaged coherency dyadic.

\subsection{Ensemble-averaged coherency dyadic}

An instructive second moment in the field is the time-averaged (or, equivalently, ensemble-averaged) coherency dyadic, defined by the relation

$$
\begin{aligned}
\overline{\mathbf{C}}(\mathbf{r}) & =\left\langle\mathbf{E}(\mathbf{r}, t) \otimes \mathbf{E}^{\star}(\mathbf{r}, t)\right\rangle \\
& =\left\langle\mathbf{E}(\mathbf{r}) \mathrm{e}^{-\mathrm{j} \omega \mathrm{t}} \otimes\left[\mathbf{E}(\mathbf{r}) \mathrm{e}^{-\mathrm{j} \omega t}\right]^{\star}\right\rangle \\
& =\left\langle\mathbf{E}(\mathbf{r}) \otimes \mathbf{E}^{\star}(\mathbf{r})\right\rangle .
\end{aligned}
$$

Representing the total field $\mathbf{E}$ as the sum of the coherent field $\mathbf{E}_{\mathrm{c}}$ and the diffuse (incoherent) scattered field $\mathscr{E}_{\text {sct }}$,

$\mathbf{E}=\mathbf{E}_{0}+\mathbf{E}_{\text {sct }}=\mathbf{E}_{0}+\left\langle\mathbf{E}_{\mathrm{sct}}\right\rangle+\mathscr{E}_{\text {sct }}=\mathbf{E}_{\mathrm{c}}+\mathscr{E}_{\text {sct }}$,

with (cf. Eq. (9))

$\mathscr{E}_{\mathrm{sct}}=\mathbf{E}_{\mathrm{sct}}-\left\langle\mathbf{E}_{\mathrm{sct}}\right\rangle=\sum_{i} \mathbf{E}_{\mathrm{sct} i}-\sum_{i}\left\langle\mathbf{E}_{\mathrm{sct} i}\right\rangle$,

and taking into account that $\left\langle\mathscr{E}_{\text {sct }}\right\rangle=\mathbf{0}$, we obtain the following representation for the coherency dyadic:

$\overline{\mathbf{C}}=\mathbf{E}_{\mathrm{c}} \otimes \mathbf{E}_{\mathrm{c}}^{\star}+\left\langle\mathscr{E}_{\mathrm{sct}} \otimes \mathscr{E}_{\mathrm{sct}}^{\star}\right\rangle=\overline{\mathbf{C}}_{\mathrm{c}}+\overline{\mathscr{C}}_{\mathrm{d}}$,

where

$\overline{\mathbf{C}}_{\mathrm{c}}=\mathbf{E}_{\mathrm{c}} \otimes \mathbf{E}_{\mathrm{c}}^{\star}$

is the coherent part of the coherency dyadic, and

$\overline{\mathscr{C}}_{\mathrm{d}}=\left\langle\mathscr{E}_{\mathrm{sct}} \otimes \mathscr{E}_{\mathrm{sct}}^{\star}\right\rangle$

is the diffuse (incoherent) coherency dyadic. In view of Eq. (107), the diffuse coherency dyadic (110) can be written as

$\overline{\mathscr{C}}_{\mathrm{d}}=\overline{\mathscr{C}}_{\mathrm{dL}}+\overline{\mathscr{C}}_{\mathrm{dC}}$,

where

$\overline{\mathscr{C}}_{\mathrm{dL}}=\sum_{i}\left\langle\mathbf{E}_{\mathrm{sct} i} \otimes \mathbf{E}_{\mathrm{sct} i}^{\star}\right\rangle=n_{0} \int_{D}\left\langle\mathbf{E}_{\mathrm{sct} i} \otimes \mathbf{E}_{\mathrm{sct} i}^{\star}\right\rangle_{i} \mathrm{~d}^{3} \mathbf{R}_{i}$

is the diffuse ladder coherency dyadic, and (in general, for a dense discrete random medium characterized by the pair distribution function $g$ )

$$
\begin{aligned}
\overline{\mathscr{C}}_{\mathrm{dc}} & =\sum_{i} \sum_{j \neq i}\left\langle\mathbf{E}_{\mathrm{sct} i} \otimes \mathbf{E}_{\mathrm{sct} j}^{\star}\right\rangle-\sum_{i} \sum_{j}\left\langle\mathbf{E}_{\mathrm{scti} i}\right\rangle \otimes\left\langle\mathbf{E}_{\mathrm{sct} j}^{\star}\right\rangle \\
& =n_{0}^{2} \int_{D}\left[\left\langle\mathbf{E}_{\mathrm{sct} i} \otimes \mathbf{E}_{\mathrm{sct} j}^{\star}\right\rangle_{i j} g\left(R_{i j}\right)\right. \\
& \left.-\left\langle\mathbf{E}_{\mathrm{sct} i}\right\rangle_{i} \otimes\left\langle\mathbf{E}_{\mathrm{sct} j}^{\star}\right\rangle_{j}\right] \mathrm{d}^{3} \mathbf{R}_{j} \mathrm{~d}^{3} \mathbf{R}_{i}
\end{aligned}
$$

is the diffuse cross coherency dyadic. Finally, defining the ladder coherency dyadic by the relation

$\overline{\mathbf{C}}_{\mathrm{L}}=\overline{\mathbf{C}}_{\mathrm{c}}+\overline{\mathscr{C}}_{\mathrm{dL}}=\overline{\mathbf{C}}_{\mathrm{c}}+n_{0} \int_{D}\left\langle\mathbf{E}_{\mathrm{sct} i} \otimes \mathbf{E}_{\mathrm{sct} i}^{\star}\right\rangle_{i} \mathrm{~d}^{3} \mathbf{R}_{i}$,

yields the representation

$\overline{\mathbf{C}}=\overline{\mathbf{C}}_{\mathrm{L}}+\overline{\mathscr{C}}_{\mathrm{dC}}=\overline{\mathbf{C}}_{\mathrm{c}}+\overline{\mathscr{C}}_{\mathrm{dL}}+\overline{\mathscr{C}}_{\mathrm{dC}}$.

Thus, the coherency dyadic is written as the sum of two terms: the configuration average of the dyadic product of the field scattered by one particle $i$ in the presence of other particles (the ladder term $\overline{\mathbf{C}}_{\mathrm{L}}$ ), and the correlation of the fields scattered by two distinct particles $i$ and $j$ (the cross term $\overline{\mathscr{C}}_{\mathrm{dc}}$ ). The ladder term $\overline{\mathbf{C}}_{\mathrm{L}}$ corresponds to the incoherent part of the scattered radiation and is obtained by summing the so-called ladder diagrams in a diagrammatic representation of $\left\langle\mathbf{E} \otimes \mathbf{E}^{\star}\right\rangle$, while the cross term $\overline{\mathscr{C}}_{\mathrm{dc}}$, arising from the interference of pairs of conjugate waves propagating along the same self-avoiding scattering path but in opposite directions, corresponds to the coherent part of the scattered radiation and is obtained by summing the cyclical diagrams in a diagrammatic representation of $\left\langle\mathbf{E} \otimes \mathbf{E}^{\star}\right\rangle$.

In the present analysis, we focus only on the incoherent part of the scattered radiation since it is straightforward to justify the use of the ladder approximation in the computation of second moments in the field at observation points in the near zone of the particulate layer. Indeed, different multi-particle contributions to the total field at an observation point residing in the far zone of any particle are transverse waves that can be characterized by the corresponding cumulative phases. The extreme sensitivity of the respective complex exponential phase factors on particle positions coupled with configuration averaging invariably serves to zero out the contributions of all second-moment diagrams except those of the ladder diagrams (see, e.g., Section 8.11 of Ref. [2] or Section 18.2 of Ref. [3]).

The quantity in terms of which the radiative transfer equation will be formulated is the diffuse specific coherency dyadic $\overline{\boldsymbol{\Sigma}}_{\mathrm{dL}}$, defined through the angular spectrum decomposition

$\overline{\mathscr{C}}_{\mathrm{dL}}(\mathbf{r})=\int \overline{\boldsymbol{\Sigma}}_{\mathrm{dL}}(\mathbf{r},-\widehat{\mathbf{p}}) \mathrm{d}^{2} \widehat{\mathbf{p}}$

The components of the diffuse specific coherency dyadic are the components of the diffuse specific coherency column vector, which in turn, determine the polarization signal of a detector. Therefore, the above equation provides an important link between the radiative transfer theory $\left(\overline{\boldsymbol{\Sigma}}_{\mathrm{dL}}\right)$ and the multiple scattering theory $\left(\overline{\mathscr{C}}_{\mathrm{dL}}\right)$. According to Eq. (114), the (ladder) specific coherency dyadic $\bar{\Sigma}_{\mathrm{L}}$, defined by

$\overline{\mathbf{C}}_{\mathrm{L}}(\mathbf{r})=\int \overline{\mathbf{\Sigma}}_{\mathrm{L}}(\mathbf{r},-\widehat{\mathbf{p}}) \mathrm{d}^{2} \widehat{\mathbf{p}}$,

is related to $\overline{\boldsymbol{\Sigma}}_{\mathrm{dL}}$ by the relation

$\overline{\boldsymbol{\Sigma}}_{\mathrm{L}}(\mathbf{r},-\widehat{\mathbf{p}})=\overline{\boldsymbol{\Sigma}}_{\mathrm{dL}}(\mathbf{r},-\widehat{\mathbf{p}})+\delta(\widehat{\mathbf{p}}+\widehat{\mathbf{s}}) \overline{\mathbf{C}}_{\mathrm{c}}(\mathbf{r})$.

\subsection{Ladder approximation of the coherency dyadic}

By virtue of Eqs. (22) and (105), the coherency dyadic can be represented as a series involving all possible schemes of scattering. This series can be represented diagramatically by means of Feynman diagrams [13], which were employed in the study of wave propagation by Bourret [14], Furutsu [15], Tatarski [16] and Frisch [17]. In the ladder approximation, the Feynman diagrams illustrated in Fig. 4 can be characterized as follows [1]:

1. The upper scattering path going through different particles corresponds to $\mathbf{E}$, the lower path corresponds to its complex conjugate value $\mathbf{E}^{\star}$, and the dyadic product $\otimes$ of the two paths is considered. 


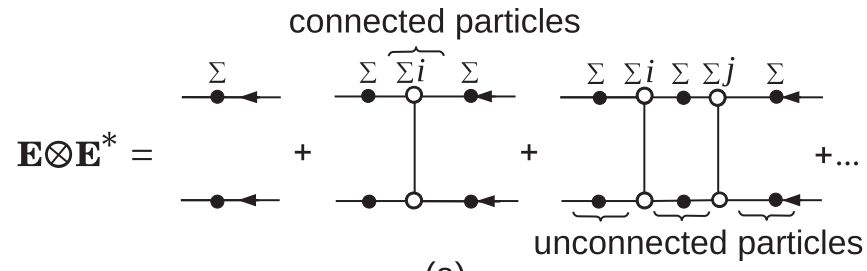

(a)

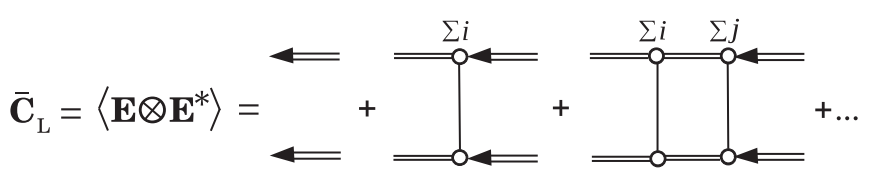

(b)

Fig. 4. (a) Diagramatic representation of $\mathbf{E} \otimes \mathbf{E}^{\star}$, and (b) the ladder coherency dyadic $\overline{\mathbf{C}}_{\mathrm{L}}=\left\langle\mathbf{E} \otimes \mathbf{E}^{\star}\right\rangle$.

2. The two paths can involve one or more common (connected) particles, and if the number of common particles is two or more, they can enter the upper and the lower path in the same order or in a different order.

3. By virtue of the Twersky approximation, neither the upper nor the lower path can go through a particle more than once, and therefore, no particles can be the origin of more than one connector.

4. In the ladder approximation, the connected particles (represented by vertical lines) are in the same order. In other words, only diagrams with vertical or no connectors are considered.

Using the diagram representation, the set of scattering schemes in the order-of-scattering series can be efficiently averaged. The configuration average over the positions of the unconnected particles, situated in the upper and the lower path between the entrance point, any connected particles and the observation point, can be computed independently (Fig. 4(a)). The result of the averaging process evidences that the source of the incoherent radiation is the coherent field $\mathbf{E}_{\mathrm{c}}$ (Fig. 4(b)). The upper (or lower) scattering path corresponds to the wave scattered by the first (right-most) connected particle which is excited by the coherent field, propagates to the second connected particle, which is again scattered and propagates to the third connected particle, and so on.

In Fig. 4(b), the symbol $\triangleleft=$ represents the coherent field, while the symbols $\circ$ and $=$ mean multiplying the coherent field by the far-field scattering dyadic $\overline{\mathbf{A}}\left(\widehat{\mathbf{r}}_{i}, \widehat{\mathbf{s}}\right)$, and by the coherent transmission dyadic $\overline{\mathbf{t}}\left(\widehat{\mathbf{r}}_{i}, r_{i}\right) / r_{i}$, respectively. The field

$\stackrel{i}{\circ} \triangleleft=\left\langle\stackrel{i}{\circ} \triangleleft-+\sum_{n} \stackrel{i}{\circ}-\stackrel{n}{\circ} \triangleleft-+\sum_{n, m, m \neq n} \stackrel{i}{\circ}-\stackrel{n}{\circ}-\stackrel{m}{\circ} \bullet-+\cdots\right\rangle$

is the coherent field at the origin of particle $i$, i.e., the configuration average sum of the incident field and the fields coming from the other particles, while the field

$$
\begin{aligned}
& =\stackrel{i}{\circ}==\left\langle-\stackrel{i}{\circ}=+\sum_{p}-\stackrel{p}{\circ}-\stackrel{i}{\circ} 4=\right. \\
& \left.+\sum_{p, q, q \neq p}-\stackrel{p}{\circ}-\stackrel{q}{\circ}-\stackrel{i}{\circ}=+\cdots\right\rangle
\end{aligned}
$$

is the configuration average of the field scattered by particle $i$, which is excited by the coherent field, and propagates through the discrete scattering medium to the observation point $\mathbf{r}$. It is important to note that in $\stackrel{i}{\circ}=$, the scattering characteristics of parti- cle $i$, excited by the coherent field, are described by the far-field scattering dyadic $\overline{\mathbf{A}}\left(\widehat{\mathbf{r}}_{i}, \widehat{\mathbf{s}}\right)$ corresponding to an excitation by a plane electromagnetic wave with wave number $k_{1}$ and propagation direction $\widehat{\mathbf{s}}$. Clearly, the use of $\overline{\mathbf{A}}\left(\widehat{\mathbf{r}}_{i}, \widehat{\mathbf{s}}\right)$ can be explained by means of Foldy's approximation, according to which, the coherent field near a particle can be analytically approximated by a plane electromagnetic wave of wavenumber $k_{1}$ (the wavenumber of the background medium) and propagation direction $\widehat{\mathbf{s}}$.

In the following, we give an analytical derivation of the ladder approximation for sparse media in order to clarify the underlying assumptions. The key point in our derivation is to consider the scattering path from the entrance point in the medium to the right-most connected particle and from this to the observation point or to another connected particle as a whole. This way of proceeding will enable us to prove all results without invoking Foldy's approximation.

Let $S$ be the set of all $N$ random scatterers. The order-ofscattering expansion allows one to represent the field at a point $\mathbf{r}$ as a sum of contributions arising from all self-avoiding scattering paths going through the particles in the set $S$. Let $A$ and $B$ be two subsets of $S$, such that the field $\mathbf{E}$ sums the contributions of all self-avoiding scattering paths connecting particles in the set $A$, while the complex conjugate field $\mathbf{E}^{\star}$ sums the contributions of all self-avoiding scattering paths connecting particles in the set $B$. The subsets $A$ and $B$ can be disjoint, they can contain one common particle, two common particles, and so on. In this regard, (i) we consider two disjoint subsets $A_{0}$ and $B_{0}$ of $S$, i.e., $A_{0} \cap B_{0}=\emptyset$, (ii) we fix a particle, say $i$, and consider two disjoint subsets $A_{i}$ and $B_{i}$ of $S_{i}=S \backslash\{i\}$, (iii) we fix two particles, say $i$ and $j$, and consider two disjoint subsets $A_{i j}$ and $B_{i j}$ of $S_{i j}=S \backslash\{i, j\}$, and so on. The direct field $\mathbf{E}$ is the sum of all scattering paths going through the particles in the sets $A_{0}, A_{i} \cup\{i\}, A_{i j} \cup\{i, j\}$, etc., while the complex conjugate field $\mathbf{E}^{\star}$ is the sum of all scattering paths going through the particles in the sets $B, B_{i} \cup\{i\}, B_{i j} \cup\{i, j\}$, etc. As the sets are disjoint by construction, the configuration average over these sets can be performed independently, i.e.,

$$
\begin{aligned}
\overline{\mathbf{C}}(\mathbf{r}) & =\sum_{A_{0}} \sum_{B_{0}}\{\mathbf{E}(\mathbf{r})\}_{A_{0}} \otimes\left\{\mathbf{E}^{\star}(\mathbf{r})\right\}_{B_{0}} \\
& +n_{0} \int_{D} \sum_{A_{i}} \sum_{B_{i}}\{\mathbf{E}(\mathbf{r})\}_{A_{i}} \otimes\left\{\mathbf{E}^{\star}(\mathbf{r})\right\}_{B_{i}} \mathrm{~d}^{3} \mathbf{R}_{i} \\
& +n_{0}^{2} \int_{D} \sum_{A_{i j}} \sum_{B_{i j}}\{\mathbf{E}(\mathbf{r})\}_{A_{i j}} \otimes\left\{\mathbf{E}^{\star}(\mathbf{r})\right\}_{B_{i j}} \mathrm{~d}^{3} \mathbf{R}_{j} \mathrm{~d}^{3} \mathbf{R}_{i} \\
& +\cdots,
\end{aligned}
$$

where

$\overline{\mathbf{C}}(\mathbf{r})=\left\langle\mathbf{E}(\mathbf{r}) \otimes \mathbf{E}^{\star}(\mathbf{r})\right\rangle:=\left\{\mathbf{E}(\mathbf{r}) \otimes \mathbf{E}^{\star}(\mathbf{r})\right\}_{S}$.

The notation $\{\mathbf{E}(\mathbf{r})\}_{A}$ stands for the configuration average of the fields corresponding to all self-avoiding scattering paths going through the particles in the set $A$, and being taken over the positions of the particles in the set $A$. The sets $A_{0}$ and $B_{0}$ are not unique, and the sums $\sum_{A_{0}} \sum_{B_{0}}$ involve all possible realizations of these sets. If in general, $\mathcal{P}(A)$ is a self-avoiding scattering path connecting particles in the set $A$, then for each realization of the sets $A_{0}$ and $B_{0}$, only those pairs of self-avoiding scattering paths $\left(\mathcal{P}\left(A_{0}\right), \mathcal{P}\left(B_{0}\right)\right)$ which do not appear in previous realizations of $A_{0}$ and $B_{0}$ are taken into account. With this convention we guarantee that the sums are taken over all distinct pairs of self-avoiding scattering paths. In the following, for large $N$, we approximate

$\sum_{A_{0}} \sum_{B_{0}}\{\mathbf{E}(\mathbf{r})\}_{A_{0}} \otimes\left\{\mathbf{E}^{\star}(\mathbf{r})\right\}_{B_{0}} \approx\{\mathbf{E}(\mathbf{r})\}_{S} \otimes\left\{\mathbf{E}^{\star}(\mathbf{r})\right\}_{S}$,

that is, we extend the sums over the particles in the sets $A_{0}$ and $B_{0}$ to the whole $S$. As $A_{0}$ and $B_{0}$ can be any subsets of $S$ and the sum is taken over all realizations of $A_{0}$ and $B_{0}$, we expect that the approximation error of Eq. (122) is small as $N$ tends to infinity. The same arguments also apply to the sets $A_{i}$ and $B_{i}$, in which case, the 


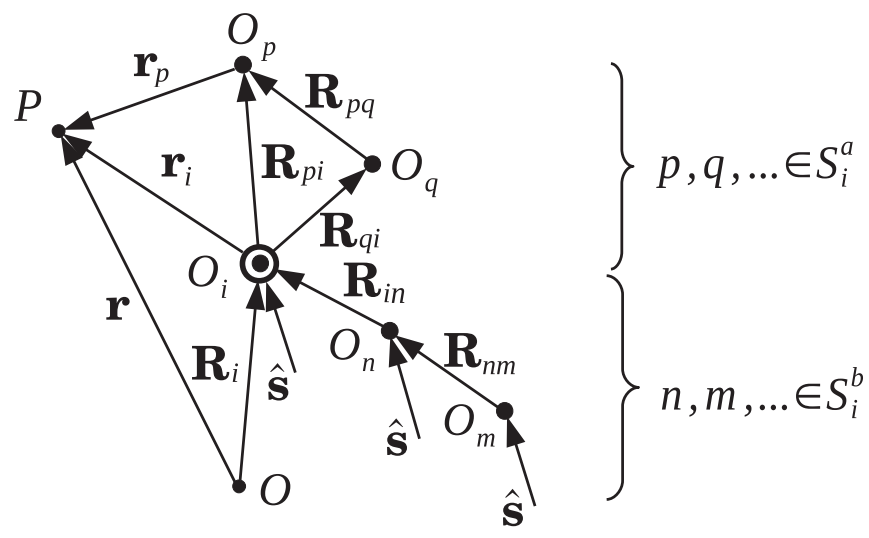

Fig. 5. Scattering paths ending at particle $i$ and connecting particle $i$ and the observation point $\mathbf{r}$.

pairs of self-avoiding scattering paths which are taken into account are of form $\left(\mathcal{P}\left(A_{i} \cup\{i\}\right), \mathcal{P}\left(B_{i} \cup\{i\}\right)\right)$. Employing the above assumption to all terms in series (121) yields

$$
\begin{aligned}
\overline{\mathbf{C}}(\mathbf{r}) & =\{\mathbf{E}(\mathbf{r})\}_{S} \otimes\left\{\mathbf{E}^{\star}(\mathbf{r})\right\}_{S}+n_{0} \int_{D}\{\mathbf{E}(\mathbf{r})\}_{S_{i}} \otimes\left\{\mathbf{E}^{\star}(\mathbf{r})\right\} S_{S_{i}} \mathrm{~d}^{3} \mathbf{R}_{i} \\
& +n_{0}^{2} \int_{D}\{\mathbf{E}(\mathbf{r})\}_{S_{i j}} \otimes\left\{\mathbf{E}^{\star}(\mathbf{r})\right\} S_{S_{i j}} \mathrm{~d}^{3} \mathbf{R}_{j} \mathrm{~d}^{3} \mathbf{R}_{i}+\cdots
\end{aligned}
$$

We are now confronted with the computation of the configuration average of the field taken over the positions of the particles in the sets $S_{i}$, $S_{i j}$, etc., that is, in subsets of $S$ with one, two, and more fixed particles. Let us consider the average field $\{\mathbf{E}(\mathbf{r})\}_{S_{i}}$, and let $S_{i}^{a}$ and $S_{i}^{b}$ be two disjoint subsets of $S_{i}$. As shown in Fig. 5 , the paths connecting the observation point $\mathbf{r}$ and particle $i$ go through all particles in the subset $S_{i}^{a}$, while the paths connecting the entrance point (the first particle struck by the incident field) and particle $i$ go through all particles in the subset $S_{i}^{b}$. By means of the Twersky approximation, the field corresponding to a direct path connecting the observation point $P$ and particle $i$ is

$\mathbf{E}^{(0)}(\mathbf{r})$

$$
\begin{aligned}
& =-\stackrel{i}{\circ}\left(\triangleleft-+\sum_{n \in S_{i}^{b}}-\stackrel{n}{\circ} \triangleleft-+\sum_{n, m \in S_{i}^{b} ; m \neq n}-\stackrel{n}{\circ}-\stackrel{m}{\circ} \triangleleft-+\cdots\right) \\
& =\overline{\mathbf{U}}\left(\mathbf{r}_{i}, \widehat{\mathbf{s}}\right) \cdot \mathbf{E}_{0}\left(\mathbf{R}_{i}\right)+\sum_{n \in S_{i}^{b}} \overline{\mathbf{U}}\left(\mathbf{r}_{i}, \widehat{\mathbf{R}_{i n}}\right) \cdot \overline{\mathbf{U}}\left(\mathbf{R}_{i n}, \widehat{\mathbf{s}}\right) \cdot \mathbf{E}_{0}\left(\mathbf{R}_{n}\right) \\
& +\sum_{n, m \in S_{i}^{b} ; m \neq n} \overline{\mathbf{U}}\left(\mathbf{r}_{i}, \widehat{\mathbf{R}}_{i n}\right) \cdot \overline{\mathbf{U}}\left(\mathbf{R}_{i n}, \widehat{\mathbf{R}}_{n m}\right) \\
& \cdot \overline{\mathbf{U}}\left(\mathbf{R}_{n m}, \widehat{\mathbf{S}}\right) \cdot \mathbf{E}_{0}\left(\mathbf{R}_{m}\right)+\cdots,
\end{aligned}
$$

the field corresponding to the single-scattering paths connecting $P$ and particle $i$ (and going through particle $p$ in Fig. 5) is

$\mathbf{E}^{(1)}(\mathbf{r})$

$$
\begin{aligned}
& =\sum_{p \in S_{i}^{a}}-\stackrel{p}{\circ}-\stackrel{i}{\circ}\left(\triangleleft-+\sum_{n \in S_{i}^{b}}-\stackrel{n}{\circ}-\right. \\
& \left.+\sum_{n, m \in S_{i}^{b} ; m \neq n}-\stackrel{n}{\circ}-\stackrel{m}{\circ}-+\ldots\right) \\
& =\sum_{p \in S_{i}^{a}} \overline{\mathbf{U}}\left(\mathbf{r}_{p}, \widehat{\mathbf{R}}_{p i}\right) \cdot \overline{\mathbf{U}}\left(\mathbf{R}_{p i}, \widehat{\mathbf{s}}\right) \cdot \mathbf{E}_{0}\left(\mathbf{R}_{i}\right)
\end{aligned}
$$

$$
\begin{aligned}
& +\sum_{p \in S_{i}^{a}} \overline{\mathbf{U}}\left(\mathbf{r}_{p}, \widehat{\mathbf{R}}_{p i}\right) \cdot \sum_{n \in S_{i}^{b}} \overline{\mathbf{U}}\left(\mathbf{R}_{p i}, \widehat{\mathbf{R}_{i n}}\right) \cdot \overline{\mathbf{U}}\left(\mathbf{R}_{i n}, \widehat{\mathbf{s}}\right) \cdot \mathbf{E}_{0}\left(\mathbf{R}_{n}\right) \\
& +\sum_{p \in S_{i}^{a}} \overline{\mathbf{U}}\left(\mathbf{r}_{p}, \widehat{\mathbf{R}}_{p i}\right) \cdot \sum_{n, m \in S_{i}^{b} ; m \neq n} \overline{\mathbf{U}}\left(\mathbf{R}_{p i}, \widehat{\mathbf{R}}_{i n}\right) \\
& \cdot \overline{\mathbf{U}}\left(\mathbf{R}_{i n}, \widehat{\mathbf{R}}_{n m}\right) \cdot \overline{\mathbf{U}}\left(\mathbf{R}_{n m}, \widehat{\mathbf{s}}\right) \cdot \mathbf{E}_{0}\left(\mathbf{R}_{m}\right)+\cdots,
\end{aligned}
$$

and so on. First, we take the configuration average over the positions of the particles in the set $S_{i}^{b}$. Extending the sums over the particles in the set $S_{i}^{b}$ (particles $n$ and $m$ in Fig. 5) to the whole set $S$, we obtain

$$
\begin{aligned}
& \sum_{S_{i}^{b}}\left\{\mathbf{E}^{(0)}(\mathbf{r})\right\}_{S_{i}^{b}}=-\stackrel{i}{\circ}\left\langle\triangleleft-+\sum_{n}-\stackrel{n}{\circ} \triangleleft-\right. \\
& \left.+\sum_{n, m ; m \neq n}-\stackrel{n}{\circ}-\stackrel{m}{\circ} \triangleleft-+\cdots\right\rangle \\
& =-\stackrel{i}{\circ} 4= \\
& =\overline{\mathbf{U}}\left(\mathbf{r}_{i}, \widehat{\mathbf{s}}\right) \cdot \mathbf{E}_{\mathrm{c}}\left(\mathbf{R}_{i}\right) \\
& \text { and } \\
& \sum_{S_{i}^{b}}\left\{\mathbf{E}^{(1)}(\mathbf{r})\right\}_{S_{i}^{b}}=\sum_{p \in S_{i}^{a}}-\stackrel{p}{\circ}-\stackrel{i}{\circ}\left\langle\triangleleft-+\sum_{n}-\stackrel{n}{\circ} \triangleleft-\right. \\
& \left.+\sum_{n, m ; m \neq n}-\stackrel{n}{\circ}-\stackrel{m}{\circ} \triangleleft-+\cdots\right\rangle \\
& =\sum_{p \in S_{i}^{a}}-\stackrel{p}{\circ}-\stackrel{i}{\circ} 4= \\
& =\sum_{p \in S_{i}^{a}} \overline{\mathbf{U}}\left(\mathbf{r}_{p}, \widehat{\mathbf{R}}_{p i}\right) \cdot \overline{\mathbf{U}}\left(\mathbf{R}_{p i}, \widehat{\mathbf{S}}\right) \cdot \mathbf{E}_{c}\left(\mathbf{R}_{i}\right) .
\end{aligned}
$$

The configuration average of the total field taken over the positions of the particles in the set $S_{i}^{b}$ is then

$$
\begin{aligned}
& \{\mathbf{E}(\mathbf{r})\}_{S_{i}^{b}}=-\stackrel{i}{\circ}=+\sum_{p \in S_{i}^{a}}-\stackrel{p}{\circ}-\stackrel{i}{\circ} \mathbf{=} \\
& +\sum_{p, q \in S_{i}^{a} ; q \neq p}-\stackrel{p}{\circ}-\stackrel{q}{\circ}-\stackrel{i}{\circ}=+\cdots,
\end{aligned}
$$

and what is left is the computation of the configuration average taken over the positions of the particles in the set $S_{i}^{a}$, that is,

$$
\begin{aligned}
\{\mathbf{E}(\mathbf{r})\}_{S_{i}}= & \sum_{S_{i}^{a}}\left\langle\stackrel{i}{\circ}=+\sum_{p \in S_{i}^{a}}-\stackrel{p}{\circ}-\stackrel{i}{\circ}=\right. \\
& \left.+\sum_{p, q \in S_{i}^{a} ; q \neq p}-\stackrel{p}{\circ}-\stackrel{q}{\circ}-\stackrel{i}{\circ}=+\cdots\right\rangle .
\end{aligned}
$$

To do this, we extend the sums over the particles in the set $S_{i}^{a}$ (particles $p$ and $q$ in Fig. 5) to the whole set $S$, giving

$$
\begin{aligned}
&\{\mathbf{E}(\mathbf{r})\}_{S_{i}}=\left\langle-\stackrel{i}{\circ}=+\sum_{p}-\stackrel{p}{\circ}-\stackrel{i}{\circ} \mathbf{=}\right. \\
&\left.+\sum_{p \cdot q ; q \neq p}-\stackrel{p}{\circ}-\stackrel{q}{\circ}-\stackrel{i}{\circ}=+\cdots\right\rangle \\
&=\left\langle\overline{\mathbf{U}}\left(\mathbf{r}_{i}, \widehat{\mathbf{s}}\right) \cdot \mathbf{E}_{\mathrm{c}}\left(\mathbf{R}_{i}\right)\right.
\end{aligned}
$$




$$
\left.+\sum_{p} \overline{\mathbf{U}}\left(\mathbf{r}_{p}, \widehat{\mathbf{R}}_{p i}\right) \cdot \overline{\mathbf{U}}\left(\mathbf{R}_{p i}, \widehat{\mathbf{s}}\right) \cdot \mathbf{E}_{c}\left(\mathbf{R}_{i}\right)+\cdots\right),
$$

and use the stationary phase method for computing integrals of the form [12]

$I=\int_{-\infty}^{\infty} \int_{-\infty}^{\infty} g(x, y) \frac{\mathrm{e}^{\mathrm{j}_{1}\left(\mathrm{r}_{1}+\mathrm{r}_{2}\right)}}{r_{1} r_{2}} \mathrm{~d} x \mathrm{~d} y$,

where

$r_{1}=r_{1}(x, y)=\sqrt{x^{2}+y^{2}+z^{2}}$,

$r_{2}=r_{2}(x, y)=\sqrt{(X-x)^{2}+(Y-y)^{2}+(Z-z)^{2}}$.

Note that for the integral (127), the stationary phase point $\left(x_{\mathrm{s}}, y_{\mathrm{s}}\right)$ of the function $f(x, y)=r_{1}(x, y)+r_{2}(x, y)$ is

$\frac{x_{\mathrm{s}}}{X}=\frac{y_{\mathrm{s}}}{Y}=\frac{r_{1 \mathrm{~s}}}{r_{1 \mathrm{~s}}+r_{2 \mathrm{~s}}}$,

and we have

$I \approx \mathrm{j}_{\mathrm{k}_{1} \mathrm{Z}_{\mathrm{s}}} \mathrm{g}\left(\mathrm{x}_{\mathrm{s}}, \mathrm{y}_{\mathrm{s}}\right) \mathrm{e}^{\mathrm{jk}\left(\mathrm{r}_{1 \mathrm{~s}}+\mathrm{r}_{2 \mathrm{~s}}\right)}$,

with $r_{1 \mathrm{~s}}=r_{1}\left(x_{\mathrm{s}}, y_{\mathrm{s}}\right), r_{2 \mathrm{~s}}=r_{2}\left(x_{\mathrm{s}}, y_{\mathrm{s}}\right)$, and

$Z_{\mathrm{s}}=|z|+|Z-z|= \begin{cases}Z+2|z|, Z<0 \\ Z, & 0<Z<Z \\ 2 z-Z, & z>Z\end{cases}$

If $X=Y=0$, the stationary point is $x_{\mathrm{s}}=y_{\mathrm{s}}=0$, and from Eqs. (128)-(129), we see that $r_{1 \mathrm{~s}}+r_{2 \mathrm{~s}}=|z|+|Z-z|=Z_{\mathrm{s}}$. For example, to compute the second term in Eq. (126), we use the Cartesian coordinate system centered at $O_{i}$ and with the $z$-axis directed along the vector $\mathbf{r}_{i}$. Application of the stationary phase method then yields

$$
\begin{aligned}
& \sum_{p}\left\langle\overline{\mathbf{U}}\left(\mathbf{r}_{p}, \widehat{\mathbf{R}}_{p i}\right) \cdot \overline{\mathbf{U}}\left(\mathbf{R}_{p i}, \widehat{\mathbf{s}}\right) \cdot \mathbf{E}_{\mathrm{c}}\left(\mathbf{R}_{i}\right)\right\rangle \\
& =n_{0} \int\left[\int_{-\infty}^{\infty} \int_{-\infty}^{\infty} \frac{\mathrm{e}^{\mathrm{j} \mathrm{k}_{1}\left(\mathrm{r}_{\mathrm{p}}+\mathrm{R}_{\mathrm{pi}}\right)}}{r_{p} R_{p i}} \overline{\mathbf{A}}\left(\widehat{\mathbf{r}}_{p}, \widehat{\mathbf{R}}_{p i}\right)\right. \\
& \left.\cdot \overline{\mathbf{A}}\left(\widehat{\mathbf{R}}_{p i}, \widehat{\mathbf{s}}\right) \cdot \mathbf{E}_{\mathrm{c}}\left(\mathbf{R}_{i}\right) \mathrm{d} x_{p i} \mathrm{~d} y_{p i}\right] \mathrm{d} z_{p i} \\
& =n_{0} \mathrm{j} \frac{2 \pi}{\mathrm{k}_{1}} \overline{\mathbf{A}}\left(\widehat{\mathbf{r}}_{\mathrm{i}}, \widehat{\mathbf{r}}_{\mathrm{i}}\right) \cdot \overline{\mathbf{A}}\left(\widehat{\mathbf{r}}_{\mathrm{i}}, \widehat{\mathbf{s}}\right) \cdot \mathbf{E}_{\mathrm{c}}\left(\mathbf{R}_{\mathrm{i}}\right) \frac{\mathrm{e}^{\mathrm{j} \mathrm{k}_{1} \mathrm{r}_{\mathrm{i}}}}{\mathrm{z}_{\mathrm{i}}} \int_{0}^{\mathrm{z}_{\mathrm{i}}} \mathrm{d} z_{\mathrm{pi}} \\
& =\frac{\mathrm{e}^{\mathrm{j} \mathrm{k}_{1} \mathrm{r}_{\mathrm{i}}}}{r_{i}} \mathrm{j} \frac{2 \pi}{\mathrm{k}_{1}} \mathrm{n}_{0} \mathrm{r}_{\mathrm{i}} \overline{\mathbf{A}}\left(\widehat{\mathbf{r}}_{\mathrm{i}}, \widehat{\mathbf{r}}_{\mathrm{i}}\right) \cdot \overline{\mathbf{A}}\left(\widehat{\mathbf{r}}_{\mathrm{i}}, \widehat{\mathbf{s}}\right) \cdot \mathbf{E}_{c}\left(\mathbf{R}_{\mathrm{i}}\right),
\end{aligned}
$$

where $\left(0,0, z_{i}\right)$ and $\left(x_{p i}, y_{p i}, z_{p i}\right)$ are the Cartesian coordinates of the points $P$ and $O_{p}$ in the coordinate system of particle $i$, respectively. In deriving Eq. (133), we considered only the contribution of the interval $0<z_{p i}<z_{i}$, and the fact that in the chosen coordinate system, $r_{i}=z_{i}$. Note that for $z_{p i}>z_{i}$ and $z_{p i}<0$ in Eq. (132), we obtain integrals which can be neglected. For example, in the case $z_{p i}>z_{i}$, we have $Z_{\mathrm{s}}=2 z_{p i}-z_{i}$, and the integral over $z_{p i}$ in Eq. (133) is

$$
\begin{aligned}
I\left(z_{i}, z_{B i}\right) & =\mathrm{j} \frac{2 \pi}{\mathrm{k}_{1}} \int_{\mathrm{z}_{\mathrm{i}}}^{\mathrm{z}_{\mathrm{Bi}}} \frac{\mathrm{e}^{\mathrm{j} \mathrm{k}_{1}\left(2 \mathrm{z}_{\mathrm{pi}}-\mathrm{z}_{\mathrm{i}}\right)}}{2 \mathrm{z}_{\mathrm{pi}}-\mathrm{z}_{\mathrm{i}}} \mathrm{d} \mathrm{z}_{\mathrm{pi}} \\
& =\mathrm{j} \frac{\pi}{\mathrm{k}_{1}}\left[\int_{\mathrm{k}_{1} \mathrm{z}_{\mathrm{i}}}^{\infty} \frac{\mathrm{e}^{\mathrm{jt}}}{\mathrm{t}} \mathrm{dt}-\int_{\mathrm{k}_{1}\left(2 \mathrm{z}_{\mathrm{Bi}}-\mathrm{z}_{\mathrm{i}}\right)}^{\infty} \frac{\mathrm{e}^{\mathrm{jt}}}{\mathrm{t}} \mathrm{dt}\right] \\
& =\mathrm{j} \frac{\pi}{\mathrm{k}_{1}}\left[\operatorname{Ei}\left(\mathrm{k}_{1} \mathrm{z}_{\mathrm{i}}\right)-\operatorname{Ei}\left(\mathrm{k}_{1}\left(2 \mathrm{z}_{\mathrm{Bi}}-\mathrm{z}_{\mathrm{i}}\right)\right)\right] .
\end{aligned}
$$

Here, $\left(0,0, z_{B i}\right)$ are the Cartesian coordinates of the point $B$, the intersection point of the $z$-axis and the upper layer boundary, in the coordinate system of particle $i$, and $\operatorname{Ei}(x)$ is the modified exponential integral,

$$
\operatorname{Ei}(x)=\int_{x}^{\infty} \frac{\mathrm{e}^{\mathrm{j} t}}{t} \mathrm{~d} t
$$

For large values of the argument, $\operatorname{Ei}(x)$ assumes the series representation (asymptotic expansion)

$$
\mathrm{Ei}(x)=\mathrm{j} \frac{\mathrm{e}^{\mathrm{j} x}}{\mathrm{x}}\left(1-\frac{2 !}{\mathrm{x}^{2}}+\frac{4 !}{\mathrm{x}^{4}}-\cdots\right)+\frac{\mathrm{e}^{\mathrm{j} \mathrm{x}}}{\mathrm{x}}\left(\frac{1}{\mathrm{x}}-\frac{3 !}{\mathrm{x}^{3}}+\frac{5 !}{\mathrm{x}^{5}}-\cdots\right),
$$

so that, by taking into account that $k_{1} z_{i}<k_{1}\left(2 z_{B i}-z_{i}\right)$, we infer that for large values of $k_{1} z_{i}$, the integral (134) behaves as $O\left(1 / k_{1} z_{i}\right)$. Thus, if the observation point is in the far-field region of particle $i$, the integral (134) vanishes. The configuration average over the positions of the particles in the set $S_{i}$ is then

$$
\begin{aligned}
\{\mathbf{E}(\mathbf{r})\}_{S_{i}} & =\frac{\mathrm{e}^{\mathrm{j} \mathrm{k}_{1} \mathrm{r}_{\mathrm{i}}}}{r_{i}}\left[\overline{\mathbf{I}}+\mathrm{j} \frac{2 \pi}{\mathrm{k}_{1}} \mathrm{n}_{0} \mathrm{r}_{\mathrm{i}} \overline{\mathbf{A}}\left(\widehat{\mathbf{r}}_{\mathrm{i}}, \widehat{\mathbf{r}}_{\mathrm{i}}\right)+\cdots\right] \cdot \overline{\mathbf{A}}\left(\widehat{\mathbf{r}}_{\mathrm{i}}, \widehat{\mathbf{s}}\right) \cdot \mathbf{E}_{\mathrm{c}}\left(\mathbf{R}_{\mathrm{i}}\right) \\
& =\frac{1}{r_{i}} \exp \left\{\mathrm{j}\left[\mathrm{k}_{1} \overline{\mathbf{I}}+\frac{2 \pi}{\mathrm{k}_{1}} \mathrm{n}_{0} \overline{\mathbf{A}}\left(\widehat{\mathbf{r}}_{\mathrm{i}}, \widehat{\mathbf{r}}_{\mathrm{i}}\right)\right] \mathrm{r}_{\mathrm{i}}\right\} \cdot \overline{\mathbf{A}}\left(\widehat{\mathbf{r}_{i}}, \widehat{\mathbf{s}}\right) \cdot \mathbf{E}_{\mathrm{c}}\left(\mathbf{R}_{\mathrm{i}}\right) \\
& =\overline{\mathbf{V}}\left(\mathbf{r}_{i}, \widehat{\mathbf{s}}\right) \cdot \mathbf{E}_{\mathrm{c}}\left(\mathbf{R}_{i}\right),
\end{aligned}
$$

with

$\overline{\mathbf{V}}\left(\mathbf{r}_{i}, \widehat{\mathbf{s}}\right)=\frac{\overline{\mathbf{t}}\left(\widehat{\mathbf{r}}_{i}, r_{i}\right)}{r_{i}} \cdot \overline{\mathbf{A}}\left(\widehat{\mathbf{r}}_{i}, \widehat{\mathbf{s}}\right)$.

Thus, we have shown that

$$
\{\mathbf{E}(\mathbf{r})\}_{S_{i}} \otimes\left\{\mathbf{E}^{\star}(\mathbf{r})\right\}_{S_{i}}=\overline{\mathbf{V}}\left(\mathbf{r}_{i}, \widehat{\mathbf{s}}\right) \cdot \overline{\mathbf{C}}_{\mathrm{c}}\left(\mathbf{R}_{i}\right) \cdot \overline{\mathbf{V}}^{\dagger}\left(\mathbf{r}_{i}, \widehat{\mathbf{s}}\right) .
$$

To compute the configuration average with two fixed particles we proceed analogously and obtain

$$
\{\mathbf{E}(\mathbf{r})\}_{s_{i j}}=\overline{\mathbf{V}}\left(\mathbf{r}_{i}, \widehat{\mathbf{R}}_{i j}\right) \cdot \overline{\mathbf{V}}\left(\mathbf{R}_{i j}, \widehat{\mathbf{s}}\right) \cdot \mathbf{E}_{c}\left(\mathbf{R}_{j}\right) \text {. }
$$

In the ladder approximation for the coherency dyadic the order of particles $i$ and $j$ in the expressions for $\{\mathbf{E}\}_{S_{i j}}$ and $\left\{\mathbf{E}^{\star}\right\}_{S_{i j}}$ is considered to be the same. In other words, the ordered set of connected particles $\{i, j\}$ is associated with both the direct and the complexconjugate field. We obtain

$$
\begin{aligned}
\{\mathbf{E}(\mathbf{r})\}_{S_{i j}} \otimes\left\{\mathbf{E}^{\star}(\mathbf{r})\right\}_{S_{i j}} & =\overline{\mathbf{V}}\left(\mathbf{r}_{i}, \widehat{\mathbf{R}}_{i j}\right) \cdot \overline{\mathbf{V}}\left(\mathbf{R}_{i j}, \widehat{\mathbf{s}}\right) \\
& \cdot \overline{\mathbf{C}}_{\mathrm{c}}\left(\mathbf{R}_{j}\right) \cdot \overline{\mathbf{V}}^{\dagger}\left(\mathbf{R}_{i j}, \widehat{\mathbf{s}}\right) \cdot \overline{\mathbf{V}}^{\dagger}\left(\mathbf{r}_{i}, \widehat{\mathbf{R}}_{i j}\right) .
\end{aligned}
$$

Note that when averaging over the positions of particles $i$ and $j$, the term corresponding to the ordered set $\{j, i\}$ (the reverse order of particles $i$ and $j$ ) is much smaller than the term corresponding to the ordered set $\{i, j\}$. More specifically, the contribution of the term

$$
\begin{aligned}
& \overline{\mathbf{V}}\left(\mathbf{r}_{i}, \widehat{\mathbf{R}}_{i j}\right) \cdot \overline{\mathbf{V}}\left(\mathbf{R}_{i j}, \widehat{\mathbf{s}}\right) \cdot\left[\mathbf{E}_{c}\left(\mathbf{R}_{j}\right) \otimes \mathbf{E}_{c}^{\star}\left(\mathbf{R}_{i}\right)\right] \\
& \cdot \overline{\mathbf{V}}^{\dagger}\left(\mathbf{R}_{j i}, \widehat{\boldsymbol{s}}\right) \cdot \overline{\mathbf{V}}^{\dagger}\left(\mathbf{r}_{j}, \widehat{\mathbf{R}}_{j i}\right)
\end{aligned}
$$

is much smaller than that of the term

$$
\begin{aligned}
& \overline{\mathbf{V}}\left(\mathbf{r}_{i}, \widehat{\mathbf{R}}_{i j}\right) \cdot \overline{\mathbf{V}}\left(\mathbf{R}_{i j}, \widehat{\mathbf{s}}\right) \cdot\left[\mathbf{E}_{c}\left(\mathbf{R}_{j}\right) \otimes \mathbf{E}_{c}^{*}\left(\mathbf{R}_{j}\right)\right] \\
& \cdot \overline{\mathbf{V}}^{\dagger}\left(\mathbf{R}_{i j}, \widehat{\mathbf{s}}\right) \cdot \overline{\mathbf{V}}^{\dagger}\left(\mathbf{r}_{i}, \widehat{\mathbf{R}}_{i j}\right) .
\end{aligned}
$$

This statement has been justified as follows. Using Eq. (138), the result

$$
\frac{\overline{\mathbf{t}}\left(\widehat{\mathbf{r}}_{i}, r_{i}\right)}{r_{i}}=\frac{\mathrm{e}^{\mathrm{j} \mathrm{k}_{1} \mathrm{r}_{\mathrm{i}}}}{r_{i}} \exp \left[\mathrm{j} \frac{2 \pi}{\mathrm{k}_{1}} \mathrm{n}_{0} \mathrm{r}_{\mathrm{i}} \overline{\mathbf{A}}\left(\widehat{\mathbf{r}}_{\mathrm{i}}, \widehat{\mathbf{r}}_{\mathrm{i}}\right)\right] \text {, }
$$

and the approximation $s\left(\mathbf{R}_{i},-\widehat{\mathbf{s}}\right) \approx s\left(\mathbf{R}_{j},-\widehat{\mathbf{s}}\right)+\widehat{\mathbf{s}} \cdot \mathbf{R}_{i j}$, we find that Eq. (142) contains the additional exponential factor $\exp \left[j k_{1}\left(r_{i}-\right.\right.$ $\left.\mathrm{r}_{\mathrm{j}}-\widehat{\mathbf{s}} \cdot \mathbf{R}_{\mathrm{ij}}\right)$ ]. This rapidly oscillating factor causes the contribution of this cyclic term to vanish upon averaging over the positions of particles $i$ and $j$. The contribution of the cyclical diagrams 
becomes comparable to the contribution of the ladder diagrams when the observation point is in the far-field region of the scattering medium and is in the direction opposite to the direction of incidence $(\widehat{\mathbf{r}}=-\widehat{\mathbf{s}})$. In the limit $r \rightarrow \infty$, we have $r_{j}=r_{i}-\widehat{\mathbf{r}} \cdot \mathbf{R}_{j i}=$ $r_{i}-\widehat{\mathbf{s}} \cdot \mathbf{R}_{i j}$, and the exponential factor becomes identically equal to unity. Strictly speaking, this never happens to an infinite particulate layer.

Collecting all results, we arrive at

$$
\begin{aligned}
\overline{\mathbf{C}}_{\mathrm{L}}(\mathbf{r}) & =\overline{\mathbf{C}}_{\mathrm{c}}(\mathbf{r})+n_{0} \int_{D} \overline{\mathbf{V}}\left(\mathbf{r}_{i}, \widehat{\mathbf{s}}\right) \cdot \overline{\mathbf{C}}_{\mathrm{c}}\left(\mathbf{R}_{i}\right) \cdot \overline{\mathbf{V}}^{\dagger}\left(\mathbf{r}_{i}, \widehat{\mathbf{s}}\right) \mathrm{d}^{3} \mathbf{R}_{i} \\
& +n_{0}^{2} \int_{D} \overline{\mathbf{V}}\left(\mathbf{r}_{i}, \widehat{\mathbf{R}}_{i j}\right) \cdot \overline{\mathbf{V}}\left(\mathbf{R}_{i j}, \widehat{\mathbf{s}}\right) \cdot \overline{\mathbf{C}}_{\mathrm{c}}\left(\mathbf{R}_{j}\right) \cdot \overline{\mathbf{V}}^{\dagger}\left(\mathbf{R}_{i j}, \widehat{\mathbf{s}}\right) \\
& \cdot \overline{\mathbf{V}}^{\dagger}\left(\mathbf{r}_{i}, \widehat{\mathbf{R}}_{i j}\right) \mathrm{d}^{3} \mathbf{R}_{j} \mathrm{~d}^{3} \mathbf{R}_{i}+\cdots
\end{aligned}
$$

In conclusion, our analysis shows that

1. the ladder approximation depicted in Fig. 4(b) includes only diagrams with vertical or no connectors,

2. the expression of the field $₫=$, representing the configuration average of the wave scattered by particle $i$ and propagating through the effective medium to the observation point $\mathbf{r}$, is given by Eqs. (137) and (138), and

3. the scattering characteristics of particle $i$, excited by the coherent field propagating in the direction $\widehat{\mathbf{s}}$, are described by the far-field scattering dyadic $\overline{\mathbf{A}}\left(\widehat{\mathbf{r}}_{i}, \widehat{\mathbf{s}}\right)$.

\subsection{Vector radiative transfer equation}

From Eqs. (117) and (145), the specific coherency dyadic is found to be

$$
\begin{aligned}
\overline{\mathbf{\Sigma}}_{\mathrm{L}}(\mathbf{r},-\widehat{\mathbf{p}}) & =\delta(\widehat{\mathbf{p}}+\widehat{\mathbf{s}}) \overline{\mathbf{C}}_{\mathrm{c}}(\mathbf{r})+n_{0} \int \overline{\mathbf{t}}(-\widehat{\mathbf{p}}, p) \cdot \overline{\mathbf{A}}(-\widehat{\mathbf{p}}, \widehat{\mathbf{s}}) \\
& \cdot \overline{\mathbf{C}}_{\mathrm{c}}\left(\mathbf{R}_{i}\right) \cdot \overline{\mathbf{A}}^{\dagger}(-\widehat{\mathbf{p}}, \widehat{\mathbf{s}}) \cdot \overline{\mathbf{t}}^{\dagger}(-\widehat{\mathbf{p}}, p) \mathrm{d} p \\
& +n_{0}^{2} \int \overline{\mathbf{t}}(-\widehat{\mathbf{p}}, p) \cdot \overline{\mathbf{A}}\left(-\widehat{\mathbf{p}},-\widehat{\mathbf{R}}_{j i}\right) \cdot \overline{\mathbf{t}}\left(-\widehat{\mathbf{R}}_{j i}, R_{j i}\right) \\
& \cdot \overline{\mathbf{A}}\left(-\widehat{\mathbf{R}}_{j i}, \widehat{\mathbf{s}}\right) \cdot \overline{\mathbf{C}}_{\mathrm{c}}\left(\mathbf{R}_{j}\right) \cdot \overline{\mathbf{A}}^{\dagger}\left(-\widehat{\mathbf{R}}_{j i}, \widehat{\mathbf{s}}\right) \cdot \overline{\mathbf{t}}^{\dagger}\left(-\widehat{\mathbf{R}}_{j i}, R_{j i}\right) \\
& \cdot \overline{\mathbf{A}}^{\dagger}\left(-\widehat{\mathbf{p}},-\widehat{\mathbf{R}}_{j i}\right) \overline{\mathbf{t}}^{\dagger}(-\widehat{\mathbf{p}}, p) \mathrm{d} R_{j i} \mathrm{~d}^{2} \widehat{\mathbf{R}}_{j i} \mathrm{~d} p+\cdots,
\end{aligned}
$$

where the relevant quantities for integral calculations are illustrated in Fig. 2. The series (146) is the expanded form of the integral equation

$$
\begin{aligned}
\overline{\mathbf{\Sigma}}_{\mathrm{L}}(\mathbf{r},-\widehat{\mathbf{p}}) & =\delta(\widehat{\mathbf{p}}+\widehat{\mathbf{s}}) \overline{\mathbf{C}}_{\mathrm{c}}(\mathbf{r})+n_{0} \int \overline{\mathbf{t}}(-\widehat{\mathbf{p}}, p) \cdot \overline{\mathbf{A}}\left(-\widehat{\mathbf{p}},-\widehat{\mathbf{p}}^{\prime}\right) \\
& \cdot \overline{\boldsymbol{\Sigma}}_{\mathrm{L}}\left(\mathbf{r}+\mathbf{p},-\widehat{\mathbf{p}}^{\prime}\right) \cdot \overline{\mathbf{A}}^{\dagger}\left(-\widehat{\mathbf{p}},-\widehat{\mathbf{p}}^{\prime}\right) \cdot \overline{\mathbf{t}}^{\dagger}(-\widehat{\mathbf{p}}, p) \mathrm{d}^{2} \widehat{\mathbf{p}}^{\prime} \mathrm{d} p,
\end{aligned}
$$

which, with the notation of Fig. $2, s=s(\mathbf{r}, \widehat{\mathbf{p}})$, and $\widehat{\mathbf{q}}=-\widehat{\mathbf{p}}$, can be expressed in a more familiar form as

$$
\begin{aligned}
\overline{\boldsymbol{\Sigma}}_{\mathrm{L}}(s, \widehat{\mathbf{q}}) & =\delta(\widehat{\mathbf{q}}-\widehat{\mathbf{s}}) \overline{\mathbf{C}}_{\mathrm{c}}(s)+n_{0} \int_{0}^{s}\left[\int \overline{\mathbf{t}}\left(\widehat{\mathbf{q}}, s-s^{\prime}\right) \cdot \overline{\mathbf{A}}\left(\widehat{\mathbf{q}}, \widehat{\mathbf{q}}^{\prime}\right)\right. \\
& \left.\cdot \overline{\boldsymbol{\Sigma}}_{\mathrm{L}}\left(s^{\prime}, \widehat{\mathbf{q}}^{\prime}\right) \cdot \overline{\mathbf{A}}^{\dagger}\left(\widehat{\mathbf{q}}, \widehat{\mathbf{q}}^{\prime}\right) \cdot \overline{\mathbf{t}}^{\dagger}\left(\widehat{\mathbf{q}}, s-s^{\prime}\right) \mathrm{d}^{2} \widehat{\mathbf{q}}^{\prime}\right] \mathrm{d} s^{\prime} .
\end{aligned}
$$

Differentiating Eq. (148) with respect to $s$ yields

$$
\begin{aligned}
\frac{\mathrm{d} \overline{\boldsymbol{\Sigma}}_{\mathrm{L}}(s, \widehat{\mathbf{q}})}{\mathrm{d} s} & =\mathrm{j} \overline{\mathbf{k}}(\widehat{\mathbf{q}}) \cdot \overline{\boldsymbol{\Sigma}}_{\mathrm{L}}(\mathrm{s}, \widehat{\mathbf{q}})-\mathrm{j} \overline{\boldsymbol{\Sigma}}_{\mathrm{L}}(\mathrm{s}, \widehat{\mathbf{q}}) \cdot \overline{\mathbf{k}}^{\dagger}(\widehat{\mathbf{q}}) \\
& +n_{0} \int \overline{\mathbf{A}}\left(\widehat{\mathbf{q}}, \widehat{\mathbf{q}}^{\prime}\right) \cdot \overline{\boldsymbol{\Sigma}}_{\mathrm{L}}\left(s, \widehat{\mathbf{q}}^{\prime}\right) \cdot \overline{\mathbf{A}}^{\dagger}\left(\widehat{\mathbf{q}}, \widehat{\mathbf{q}}^{\prime}\right) \mathrm{d}^{2} \widehat{\mathbf{q}}^{\prime} .
\end{aligned}
$$

For the diffuse specific coherency dyadic, defined by (cf. Eq. (118))

$\overline{\boldsymbol{\Sigma}}_{\mathrm{dL}}(s, \widehat{\mathbf{q}})=\overline{\boldsymbol{\Sigma}}_{\mathrm{L}}(s, \widehat{\mathbf{q}})-\delta(\widehat{\mathbf{q}}-\widehat{\mathbf{s}}) \overline{\mathbf{C}}_{\mathrm{c}}(s)$,

Eqs. (148) and (149) translate into

$\bar{\Sigma}_{\mathrm{dL}}(s, \widehat{\mathbf{q}})=n_{0} \int_{0}^{s} \overline{\mathbf{t}}\left(\widehat{\mathbf{q}}, s-s^{\prime}\right) \cdot \overline{\mathbf{A}}(\widehat{\mathbf{q}}, \widehat{\mathbf{s}})$

$$
\begin{aligned}
& \cdot \overline{\mathbf{C}}_{\mathrm{c}}\left(s^{\prime}\right) \cdot \overline{\mathbf{A}}^{\dagger}(\widehat{\mathbf{q}}, \widehat{\mathbf{s}}) \cdot \overline{\mathbf{t}}^{\dagger}\left(\widehat{\mathbf{q}}, s-s^{\prime}\right) \mathrm{d} s^{\prime} \\
& +n_{0} \int_{0}^{s}\left[\int \overline{\mathbf{t}}\left(\widehat{\mathbf{q}}, s-s^{\prime}\right) \cdot \overline{\mathbf{A}}\left(\widehat{\mathbf{q}}, \widehat{\mathbf{q}}^{\prime}\right)\right. \\
& \left.\cdot \overline{\boldsymbol{\Sigma}}_{\mathrm{dL}}\left(s^{\prime}, \widehat{\mathbf{q}}^{\prime}\right) \cdot \overline{\mathbf{A}}^{\dagger}\left(\widehat{\mathbf{q}}, \widehat{\mathbf{q}}^{\prime}\right) \cdot \overline{\mathbf{t}}^{\dagger}\left(\widehat{\mathbf{q}}, s-s^{\prime}\right) \mathrm{d}^{2} \widehat{\mathbf{q}}^{\prime}\right] \mathrm{d} s^{\prime}
\end{aligned}
$$

and

$$
\begin{aligned}
\frac{\mathrm{d} \overline{\boldsymbol{\Sigma}}_{\mathrm{dL}}(s, \widehat{\mathbf{q}})}{\mathrm{d} s} & =\mathrm{j} \overline{\mathbf{k}}(\widehat{\mathbf{q}}) \cdot \overline{\boldsymbol{\Sigma}}_{\mathrm{dL}}(\mathrm{s}, \widehat{\mathbf{q}})-\mathrm{j} \overline{\boldsymbol{\Sigma}}_{\mathrm{dL}}(\mathrm{s}, \widehat{\mathbf{q}}) \cdot \overline{\mathbf{k}}^{\dagger}(\widehat{\mathbf{q}}) \\
& +n_{0} \overline{\mathbf{A}}(\widehat{\mathbf{q}}, \widehat{\mathbf{s}}) \cdot \overline{\mathbf{C}}_{\mathrm{c}}(s) \cdot \overline{\mathbf{A}}^{\dagger}(\widehat{\mathbf{q}}, \widehat{\mathbf{s}}) \\
& +n_{0} \int \overline{\mathbf{A}}\left(\widehat{\mathbf{q}}, \widehat{\mathbf{q}}^{\prime}\right) \cdot \overline{\boldsymbol{\Sigma}}_{\mathrm{dL}}\left(s, \widehat{\mathbf{q}}^{\prime}\right) \cdot \overline{\mathbf{A}}^{\dagger}\left(\widehat{\mathbf{q}}, \widehat{\mathbf{q}}^{\prime}\right) \mathrm{d}^{2} \widehat{\mathbf{q}}^{\prime},
\end{aligned}
$$

respectively.

To obtain the vector radiative transfer equation we put $s \rightarrow \mathbf{r}$, and pass from the dyadic form representation (151) to a matrix form representation. For doing this, we take into account that the diffuse specific coherency dyadic $\overline{\boldsymbol{\Sigma}}_{\mathrm{dL}}(\mathbf{r}, \widehat{\mathbf{q}})$ and the coherent part of the coherency dyadic $\overline{\mathbf{C}}_{\mathrm{c}}(\mathbf{r})$ are transverse dyadics, i.e.,

$\overline{\boldsymbol{\Sigma}}_{\mathrm{dL}}(\mathbf{r}, \widehat{\mathbf{q}})=\sum_{\eta, \mu=\theta, \varphi} \Sigma_{\eta \mu}(\mathbf{r}, \widehat{\mathbf{q}}) \widehat{\boldsymbol{\eta}}(\widehat{\mathbf{q}}) \otimes \widehat{\boldsymbol{\mu}}(\widehat{\mathbf{q}})$

and

$\overline{\mathbf{C}}_{\mathrm{c}}(\mathbf{r})=\sum_{\eta, \mu=\theta, \varphi} E_{\mathrm{c} \eta}(\mathbf{r}) E_{\mathrm{c} \mu}^{\star}(\mathbf{r}) \widehat{\boldsymbol{\eta}}(\widehat{\mathbf{q}}) \otimes \widehat{\boldsymbol{\mu}}(\widehat{\mathbf{q}})$.

These representations together with the representation of the farfield scattering dyadic $\overline{\mathbf{A}}\left(\widehat{\mathbf{q}}, \widehat{\mathbf{q}}^{\prime}\right)$ in terms of the amplitude matrix $\mathrm{S}\left(\widehat{\mathbf{q}}, \widehat{\mathbf{q}}^{\prime}\right)$, give

$$
\begin{aligned}
\frac{\mathrm{d} \Sigma_{\mathrm{dL}}(\mathbf{r}, \widehat{\mathbf{q}})}{\mathrm{d} s} & =\mathrm{jk}(\widehat{\mathbf{q}}) \Sigma_{\mathrm{dL}}(\mathbf{r}, \widehat{\mathbf{q}})-\mathrm{j} \Sigma_{\mathrm{dL}}(\mathbf{r}, \widehat{\mathbf{q}}) \mathrm{k}^{\dagger}(\widehat{\mathbf{q}}) \\
& +n_{0} \mathrm{~S}(\widehat{\mathbf{q}}, \widehat{\mathbf{s}}) \mathrm{C}_{\mathrm{c}}(\mathbf{r}) \mathrm{S}^{\dagger}(\widehat{\mathbf{q}}, \widehat{\mathbf{s}}) \\
& +n_{0} \int \mathrm{s}\left(\widehat{\mathbf{q}}, \widehat{\mathbf{q}}^{\prime}\right) \Sigma_{\mathrm{dL}}\left(\mathbf{r}, \widehat{\mathbf{q}}^{\prime}\right) \mathrm{S}^{\dagger}\left(\widehat{\mathbf{q}}, \widehat{\mathbf{q}}^{\prime}\right) \mathrm{d}^{2} \widehat{\mathbf{q}}^{\prime},
\end{aligned}
$$

where $\mathrm{k}(\widehat{\mathbf{q}})$ is the propagation constant matrix. Eq. (154) can be expressed in terms of the elements of the diffuse specific coherency matrix $\Sigma_{\mathrm{dL}}(\mathbf{r}, \widehat{\mathbf{q}})$ encapsulated in the diffuse specific coherency column vector

$\mathrm{J}_{\mathrm{d}}(\mathbf{r}, \widehat{\mathbf{q}})=\frac{1}{2} \sqrt{\frac{\varepsilon_{1}}{\mu_{0}}}\left[\begin{array}{c}\Sigma_{\theta \theta}(\mathbf{r}, \widehat{\mathbf{q}}) \\ \Sigma_{\theta \varphi}(\mathbf{r}, \widehat{\mathbf{q}}) \\ \Sigma_{\varphi \theta}(\mathbf{r}, \widehat{\mathbf{q}}) \\ \Sigma_{\varphi \varphi}(\mathbf{r}, \widehat{\mathbf{q}})\end{array}\right]$

To this end, we consider the transformation $\mathcal{T}$ mapping a $2 \times 2$ matrix into a 4-dimensional vector, i.e.,

$\mathcal{T}:\left[\begin{array}{cc}x_{\theta \theta} & x_{\theta \varphi} \\ x_{\varphi \theta} & x_{\varphi \varphi}\end{array}\right] \longmapsto\left[\begin{array}{c}x_{\theta \theta} \\ x_{\theta \varphi} \\ x_{\varphi \theta} \\ x_{\varphi \varphi}\end{array}\right]$.

Then, using the transformation rules

$$
\begin{aligned}
& \frac{1}{2} \sqrt{\frac{\varepsilon_{1}}{\mu_{0}}} \mathrm{~S}(\widehat{\mathbf{q}}, \widehat{\mathbf{s}}) \mathrm{C}_{\mathrm{c}}(\mathbf{r}) \mathrm{S}^{\dagger}(\widehat{\mathbf{q}}, \widehat{\mathbf{s}}) \stackrel{T}{\longmapsto} \mathrm{Z}_{\mathrm{J}}(\widehat{\mathbf{q}}, \widehat{\mathbf{s}}) \mathrm{J}_{\mathrm{c}}(\mathbf{r}), \\
& \frac{1}{2} \sqrt{\frac{\varepsilon_{1}}{\mu_{0}}} \mathrm{~S}\left(\widehat{\mathbf{q}}, \widehat{\mathbf{q}}^{\prime}\right) \Sigma_{\mathrm{dL}}\left(\mathbf{r}, \widehat{\mathbf{q}}^{\prime}\right) \mathrm{S}^{\dagger}\left(\widehat{\mathbf{q}}, \widehat{\mathbf{q}}^{\prime}\right) \stackrel{T}{\longmapsto} \mathrm{Z}_{\mathrm{J}}\left(\widehat{\mathbf{q}}, \widehat{\mathbf{q}}^{\prime}\right) \mathrm{J}_{\mathrm{d}}\left(\mathbf{r}, \widehat{\mathbf{q}}^{\prime}\right), \\
& \frac{1}{2} \sqrt{\frac{\varepsilon_{1}}{\mu_{0}}}\left[\mathrm{j} \mathrm{k}(\widehat{\mathbf{q}}) \Sigma_{\mathrm{dL}}(\mathbf{r}, \widehat{\mathbf{q}})-\mathrm{j} \Sigma_{\mathrm{dL}}(\mathbf{r}, \widehat{\mathbf{q}}) \mathrm{k}^{\dagger}(\widehat{\mathbf{q}})\right] \stackrel{T}{\longmapsto}-n_{0} \mathrm{~K}_{\mathrm{J}}(\widehat{\mathbf{q}}) \mathrm{J}_{\mathrm{d}}(\mathbf{r}, \widehat{\mathbf{q}}),
\end{aligned}
$$

where as in Eq. (54), $\mathrm{J}_{\mathrm{c}}$ is the coherency column vector of the coherent field, $\mathrm{z}_{\mathrm{J}}$ is the coherency phase matrix, and $\mathrm{K}_{\mathrm{J}}$ is the coherency extinction matrix, a radiative transfer equation for the diffuse specific coherency column vector

$\mathrm{J}_{\mathrm{d}}(\mathbf{r}, \widehat{\mathbf{q}})=\mathrm{J}(\mathbf{r}, \widehat{\mathbf{q}})-\delta(\widehat{\mathbf{q}}-\widehat{\mathbf{s}}) \mathrm{J}_{\mathrm{c}}(\mathbf{r})$ 
is obtained. This equation is then transformed into the vector radiative transfer equation for the diffuse specific intensity column vector

$\mathrm{I}_{\mathrm{d}}=\mathrm{DJ}_{\mathrm{d}}=\frac{1}{2} \sqrt{\frac{\varepsilon_{1}}{\mu_{0}}}\left[\begin{array}{c}\Sigma_{\theta \theta}+\Sigma_{\varphi \varphi} \\ \Sigma_{\theta \theta}-\Sigma_{\varphi \varphi} \\ -\Sigma_{\theta \varphi}-\Sigma_{\varphi \theta} \\ \mathrm{j}\left(\Sigma_{\varphi \theta}-\Sigma_{\theta \varphi}\right)\end{array}\right]$,

that is,

$$
\begin{aligned}
\frac{\mathrm{dI}_{\mathrm{d}}(\mathbf{r}, \widehat{\mathbf{q}})}{\mathrm{d} s} & =-n_{0} \mathrm{~K}(\widehat{\mathbf{q}}) \mathrm{I}_{\mathrm{d}}(\mathbf{r}, \widehat{\mathbf{q}})+n_{0} \mathrm{Z}(\widehat{\mathbf{q}}, \widehat{\mathbf{s}}) \mathrm{I}_{\mathrm{c}}(\mathbf{r}) \\
& +n_{0} \int \mathrm{Z}\left(\widehat{\mathbf{q}}, \widehat{\mathbf{q}}^{\prime}\right) \mathrm{I}_{\mathrm{d}}\left(\mathbf{r}, \widehat{\mathbf{q}}^{\prime}\right) \mathrm{d}^{2} \widehat{\mathbf{q}}^{\prime},
\end{aligned}
$$

where as in Eq. (55), $I_{c}=D_{J}$ is the Stokes column vector of the coherent field, while $\mathrm{Z}=\mathrm{DZ}_{\mathrm{J}} \mathrm{D}^{-1}$ and $\mathrm{K}=\mathrm{DK}_{\mathrm{J}} \mathrm{D}^{-1}$ are the phase and the extinction matrix of a non-spherical particle in a fixed orientation.

\subsection{Ensemble-averaged Poynting-Stokes dyadic}

In classical electromagnetics, the instantaneous local directional flow of electromagnetic energy is described by the ensembleaveraged Poynting vector involving both the electric and the magnetic field at the observation point,

$\mathbf{S}(\mathbf{r})=\frac{1}{2}\left\langle\mathbf{E}(\mathbf{r}) \times \mathbf{H}^{\star}(\mathbf{r})\right\rangle$.

Unfortunately, by virtue of being a vector product of the electric and magnetic fields, the Poynting vector does not carry explicit information on the polarization state of the scattered electromagnetic field and the field itself. A more appropriate quantity having the dimension of electromagnetic energy flux and serving this purpose is the Poynting-Stokes dyadic. The Poynting-Stokes dyadic, introduced in [18], is defined as the ensemble-averaged dyadic product of the magnetic field and the complex conjugate of the electric field, that is,

$\overline{\mathbf{P}}(\mathbf{r})=\frac{1}{2}\left\langle\mathbf{H}(\mathbf{r}) \otimes \mathbf{E}^{\star}(\mathbf{r})\right\rangle$.

In the first step of our analysis we intend to establish a relationship between the specific coherency dyadic $\overline{\boldsymbol{\Sigma}}_{\mathrm{L}}$ (and so, the specific intensity column vector) and the Poynting-Stokes dyadic $\overline{\mathbf{P}}$. Considering the order-of-scattering expansion for the magnetic field

$$
\begin{aligned}
\mathbf{H}(\mathbf{r}) & =\sqrt{\frac{\varepsilon_{1}}{\mu_{0}}}\left[\widehat{\mathbf{s}} \times \mathbf{E}_{0}(\mathbf{r})+\sum_{i} \widehat{\mathbf{r}}_{i} \times \overline{\mathbf{U}}\left(\mathbf{r}_{i}, \widehat{\mathbf{s}}\right) \cdot \mathbf{E}_{0}\left(\mathbf{R}_{i}\right)\right. \\
& +\sum_{i} \sum_{j \neq i} \widehat{\mathbf{r}}_{i} \times \overline{\mathbf{U}}\left(\mathbf{r}_{i}, \widehat{\mathbf{R}}_{i j}\right) \cdot \overline{\mathbf{U}}\left(\mathbf{R}_{i j}, \widehat{\mathbf{s}}\right) \cdot \mathbf{E}_{0}\left(\mathbf{R}_{j}\right) \\
& +\sum_{i} \sum_{j \neq i} \sum_{l \neq i, j} \widehat{\mathbf{r}}_{i} \times \overline{\mathbf{U}}\left(\mathbf{r}_{i}, \widehat{\mathbf{R}}_{i j}\right) \cdot \overline{\mathbf{U}}\left(\mathbf{R}_{i j}, \widehat{\mathbf{R}}_{j l}\right) \\
& \left.\cdot \overline{\mathbf{U}}\left(\mathbf{R}_{j l}, \widehat{\mathbf{s}}\right) \cdot \mathbf{E}_{0}\left(\mathbf{R}_{l}\right)+\cdots\right],
\end{aligned}
$$

we find that in the ladder approximation, the Poynting-Stokes dyadic possesses the series representation

$$
\begin{aligned}
\overline{\mathbf{P}}(\mathbf{r}) & =\frac{1}{2} \sqrt{\frac{\varepsilon_{1}}{\mu_{0}}}\left[\widehat{\mathbf{s}} \times \overline{\mathbf{C}}_{\mathrm{c}}(\mathbf{r})+n_{0} \int_{D} \widehat{\mathbf{r}}_{i} \times \overline{\mathbf{V}}\left(\mathbf{r}_{i}, \widehat{\mathbf{s}}\right) \cdot \overline{\mathbf{C}}_{\mathrm{c}}\left(\mathbf{R}_{i}\right)\right. \\
& \cdot \overline{\mathbf{V}}^{\dagger}\left(\mathbf{r}_{i}, \widehat{\mathbf{s}}\right) \mathrm{d}^{3} \mathbf{R}_{i}+n_{0}^{2} \int_{D} \widehat{\mathbf{r}}_{i} \times \overline{\mathbf{V}}\left(\mathbf{r}_{i}, \widehat{\mathbf{R}}_{i j}\right) \cdot \overline{\mathbf{V}}\left(\mathbf{R}_{i j}, \widehat{\mathbf{s}}\right) \\
& \left.\cdot \overline{\mathbf{C}}_{\mathrm{c}}\left(\mathbf{R}_{j}\right) \cdot \overline{\mathbf{V}}^{\dagger}\left(\mathbf{R}_{i j}, \widehat{\mathbf{s}}\right) \cdot \overline{\mathbf{V}}^{\dagger}\left(\mathbf{r}_{i}, \widehat{\mathbf{R}}_{i j}\right) \mathrm{d}^{3} \mathbf{R}_{j} \mathrm{~d}^{3} \mathbf{R}_{i}+\cdots\right] .
\end{aligned}
$$

Using the series expansion for the specific coherency dyadic (which follows from Eq. (145) with $\mathbf{R}_{i}=\mathbf{r}+\mathbf{p}$, that is, with $\mathbf{p}=-\mathbf{r}_{i}$

$$
\begin{aligned}
& \text { and } \mathrm{d}^{3} \mathbf{R}_{i}= \\
& \begin{aligned}
\overline{\mathbf{\Sigma}}_{\mathrm{L}}(\mathbf{r},-\widehat{\mathbf{p}}) & =\delta(\widehat{\mathbf{p}}+\widehat{\mathrm{s}}) \overline{\mathbf{C}}_{\mathrm{c}}(\mathbf{\mathbf { p }})+n_{0} \int \overline{\mathbf{V}}\left(\mathbf{r}_{i}, \widehat{\mathbf{s}}\right) \cdot \overline{\mathbf{C}}_{\mathrm{c}}\left(\mathbf{R}_{i}\right) \\
& \cdot \overline{\mathbf{V}}^{\dagger}\left(\mathbf{r}_{i}, \widehat{\mathbf{s}}\right) p^{2} \mathrm{~d} p+n_{0}^{2} \int \overline{\mathbf{V}}\left(\mathbf{r}_{i}, \widehat{\mathbf{R}}_{i j}\right) \cdot \overline{\mathbf{V}}\left(\mathbf{R}_{i j}, \widehat{\mathbf{s}}\right) \\
& \cdot \overline{\mathbf{C}}_{\mathrm{c}}\left(\mathbf{R}_{j}\right) \cdot \overline{\mathbf{V}}^{\dagger}\left(\mathbf{R}_{i j}, \widehat{\mathbf{s}}\right) \cdot \overline{\mathbf{V}}^{\dagger}\left(\mathbf{r}_{i}, \widehat{\mathbf{R}}_{i j}\right) \mathrm{d}^{3} \mathbf{R}_{j} p^{2} \mathrm{~d} p+\cdots,
\end{aligned}
\end{aligned}
$$

and combining this result with Eq. (163) we obtain the desired relation, namely

$\overline{\mathbf{P}}(\mathbf{r})=-\frac{1}{2} \sqrt{\frac{\varepsilon_{1}}{\mu_{0}}} \int \widehat{\mathbf{p}} \times \overline{\mathbf{\Sigma}}_{\mathrm{L}}(\mathbf{r},-\widehat{\mathbf{p}}) \mathrm{d}^{2} \widehat{\mathbf{p}}$.

The first element of the specific intensity column vector I is called the specific intensity, and analogously to Eq. (158), is given by

$I(\mathbf{r},-\widehat{\mathbf{p}})=\frac{1}{2} \sqrt{\frac{\varepsilon_{1}}{\mu_{0}}}\left[\Sigma_{\theta \theta}(\mathbf{r},-\widehat{\mathbf{p}})+\Sigma_{\varphi \varphi}(\mathbf{r},-\widehat{\mathbf{p}})\right]$.

Let us derive a series expansion for the specific intensity. For doing this we introduce the single-scattering field

$\mathbf{E}_{i}\left(\mathbf{r}_{i}, \mathbf{R}_{i}\right)=\stackrel{i}{\circ} \triangleleft=r_{i} \overline{\mathbf{V}}\left(\mathbf{r}_{i}, \widehat{\mathbf{s}}\right) \cdot \mathbf{E}_{\mathrm{c}}\left(\mathbf{R}_{i}\right)$,

the double-scattering field

$\mathbf{E}_{i j}\left(\mathbf{r}_{i}, \mathbf{R}_{i j}, \mathbf{R}_{j}\right)=\stackrel{i}{=}=\stackrel{j}{\circ} \triangleleft=$

$=r_{i} R_{i j} \overline{\mathbf{V}}\left(\mathbf{r}_{i}, \widehat{\mathbf{R}}_{i j}\right) \cdot \overline{\mathbf{V}}\left(\mathbf{R}_{i j}, \widehat{\mathbf{s}}\right) \cdot \mathbf{E}_{\mathrm{c}}\left(\mathbf{R}_{j}\right)$,

and so on. In Eqs. (167) and (168), the symbol $4=$ represents the coherent field, but now and in view of the relation $r_{i} \overline{\mathbf{V}}\left(\mathbf{r}_{i}, \widehat{\mathbf{s}}\right)=\overline{\mathbf{t}}\left(\widehat{\mathbf{r}}_{i}, r_{i}\right) \cdot \overline{\mathbf{A}}\left(\widehat{\mathbf{r}}_{i}, \widehat{\mathbf{s}}\right)$, the symbols $\circ$ and $=$ mean multiplying the coherent field by the far-field scattering dyadic $\overline{\mathbf{A}}\left(\widehat{\mathbf{r}}_{i}, \widehat{\mathbf{s}}\right)$, and the coherent transmission dyadic $\overline{\mathbf{t}}\left(\widehat{\mathbf{r}}_{i}, r_{i}\right.$ ) (in contrast to $\overline{\mathbf{t}}\left(\widehat{\mathbf{r}}_{i}, r_{i}\right) / r_{i}$ appearing in Eq. (120)), respectively. Excepting the factor $r_{i}$ in Eq. (167), $\mathbf{E}_{i}\left(\mathbf{r}_{i}, \mathbf{R}_{i}\right)$ is the field scattered by particle $i$ and propagating to the observation point $\mathbf{r}=\mathbf{R}_{i}+\mathbf{r}_{i}$ through the discrete random medium when the particle is illuminated by the coherent field $\mathbf{E}_{\mathrm{c}}\left(\mathbf{R}_{i}\right)$. In terms of these fields, the coherency dyadic is written as

$$
\begin{aligned}
\overline{\mathbf{C}}_{\mathrm{L}}(\mathbf{r}) & =\overline{\mathbf{C}}_{\mathrm{c}}(\mathbf{r})+n_{0} \int_{D} \frac{1}{r_{i}^{2}} \mathbf{E}_{i}\left(\mathbf{r}_{i}, \mathbf{R}_{i}\right) \otimes \mathbf{E}_{i}^{\star}\left(\mathbf{r}_{i}, \mathbf{R}_{i}\right) \mathrm{d}^{3} \mathbf{R}_{i} \\
& +n_{0}^{2} \int_{D} \frac{1}{r_{i}^{2} R_{i j}^{2}} \mathbf{E}_{i j}\left(\mathbf{r}_{i}, \mathbf{R}_{i j}, \mathbf{R}_{j}\right) \otimes \mathbf{E}_{i j}^{\star}\left(\mathbf{r}_{i}, \mathbf{R}_{i j}, \mathbf{R}_{j}\right) \mathrm{d}^{3} \mathbf{R}_{j} \mathrm{~d}^{3} \mathbf{R}_{i} \\
& +\cdots
\end{aligned}
$$

implying

$$
\begin{aligned}
\boldsymbol{\Sigma}_{\mathrm{L}}(\mathbf{r},-\widehat{\mathbf{p}}) & =\delta(\widehat{\mathbf{p}}+\widehat{\mathbf{s}}) \overline{\mathbf{C}}_{\mathrm{c}}(\mathbf{r})+n_{0} \int \mathbf{E}_{i}\left(-\mathbf{p}, \mathbf{R}_{i}\right) \otimes \mathbf{E}_{i}^{\star}\left(-\mathbf{p}, \mathbf{R}_{i}\right) \mathrm{d} p \\
& +n_{0}^{2} \int \mathbf{E}_{i j}\left(-\mathbf{p},-\mathbf{R}_{j i}, \mathbf{R}_{j}\right) \otimes \mathbf{E}_{i j}^{\star}\left(-\mathbf{p},-\mathbf{R}_{j i}, \mathbf{R}_{j}\right) \\
& \times \mathrm{d} R_{j i} \mathrm{~d}^{2} \widehat{\mathbf{R}}_{j i} \mathrm{~d} p+\cdots,
\end{aligned}
$$

where, as usual, $\mathbf{R}_{i}=\mathbf{r}+\mathbf{p}, \mathbf{R}_{j}=\mathbf{R}_{i}+\mathbf{R}_{j i}$, etc. The fields $\mathbf{E}_{i}$, $\mathbf{E}_{i j}$, etc. are orthogonal to the direction $\widehat{\mathbf{r}}_{i}$, e.g., from $\widehat{\mathbf{r}}_{i} \cdot \overline{\mathbf{t}}\left(\widehat{\mathbf{r}}_{i}, r_{i}\right)=0$, we have $\widehat{\mathbf{r}}_{i} \cdot \mathbf{E}_{i}=0$. Moreover, assuming for example, that

$\mathbf{E}_{i}\left(\mathbf{r}_{i}, \cdot\right)=E_{i \theta}\left(\mathbf{r}_{i}, \cdot\right) \widehat{\boldsymbol{\theta}}\left(\widehat{\mathbf{r}}_{i}\right)+E_{i \varphi}\left(\mathbf{r}_{i}, \cdot\right) \widehat{\boldsymbol{\varphi}}\left(\widehat{\mathbf{r}}_{i}\right)$,

we have the following matrix-form representation for the dyadic product $\mathbf{E}_{i} \otimes \mathbf{E}_{i}^{\star}$ :

$$
\begin{aligned}
\mathbf{E}_{i}\left(\mathbf{r}_{i}, \cdot\right) \otimes \mathbf{E}_{i}^{\star}\left(\mathbf{r}_{i}, \cdot\right) & =\left|E_{i \theta}\left(\mathbf{r}_{i}, \cdot\right)\right|^{2} \widehat{\boldsymbol{\theta}}\left(\widehat{\mathbf{r}}_{i}\right) \otimes \widehat{\boldsymbol{\theta}}\left(\widehat{\mathbf{r}}_{i}\right) \\
& +\left|E_{i \varphi}\left(\mathbf{r}_{i}, \cdot\right)\right|^{2} \widehat{\boldsymbol{\varphi}}\left(\widehat{\mathbf{r}}_{i}\right) \otimes \widehat{\boldsymbol{\varphi}}\left(\widehat{\mathbf{r}}_{i}\right)
\end{aligned}
$$




$$
\begin{aligned}
& +E_{i \theta}\left(\mathbf{r}_{i}, \cdot\right) E_{i \varphi}^{*}\left(\mathbf{r}_{i}, \cdot\right) \widehat{\boldsymbol{\theta}}\left(\widehat{\mathbf{r}}_{i}\right) \otimes \widehat{\boldsymbol{\varphi}}\left(\widehat{\mathbf{r}}_{i}\right) \\
& +E_{i \varphi}\left(\mathbf{r}_{i}, \cdot\right) E_{i \theta}^{*}\left(\mathbf{r}_{i}, \cdot\right) \widehat{\boldsymbol{\varphi}}\left(\widehat{\mathbf{r}}_{i}\right) \otimes \widehat{\boldsymbol{\theta}}\left(\widehat{\mathbf{r}}_{i}\right) .
\end{aligned}
$$

Similar representations hold for all multiple-scattering terms in the series (170). Noting for example that $\left(\mathbf{p}=-\mathbf{r}_{i}\right)$

$$
\left|\mathbf{E}_{i}\left(-\mathbf{p}, \mathbf{R}_{i}\right)\right|^{2}=\left|E_{i \theta}\left(-\mathbf{p}, \mathbf{R}_{i}\right)\right|^{2}+\left|E_{i \varphi}\left(-\mathbf{p}, \mathbf{R}_{i}\right)\right|^{2},
$$

we obtain from Eq. (170) in conjunction with Eqs. (166) and (172), the following series expansion for the specific intensity:

$$
\begin{aligned}
I(\mathbf{r},-\widehat{\mathbf{p}}) & =\frac{1}{2} \sqrt{\frac{\varepsilon_{1}}{\mu_{0}}}\left[\delta(\widehat{\mathbf{p}}+\widehat{\mathbf{s}})\left|\mathbf{E}_{\mathrm{c}}(\mathbf{r})\right|^{2}+n_{0} \int\left|\mathbf{E}_{i}\left(-\mathbf{p}, \mathbf{R}_{i}\right)\right|^{2} \mathrm{~d} p\right. \\
& \left.+n_{0}^{2} \int\left|\mathbf{E}_{i j}\left(-\mathbf{p},-\mathbf{R}_{j i}, \mathbf{R}_{j}\right)\right|^{2} \mathrm{~d} R_{j i} \mathrm{~d}^{2} \widehat{\mathbf{R}}_{j i} \mathrm{~d} p+\cdots\right] .
\end{aligned}
$$

As the next step, we aim to establish a relationship between the specific intensity and the configuration-averaged Poynting vector. In the ladder approximation, the configuration-averaged Poynting vector $\mathbf{S}$ of Eq. (160) can be written in terms of the fields $\mathbf{E}_{i}, \mathbf{E}_{i j}$, etc. as

$$
\begin{aligned}
\mathbf{S}(\mathbf{r}) & =\frac{1}{2} \sqrt{\frac{\varepsilon_{1}}{\mu_{0}}}\left\{\mathbf{E}_{\mathrm{c}}(\mathbf{r}) \times\left[\widehat{\mathbf{s}} \times \mathbf{E}_{\mathrm{c}}^{\star}(\mathbf{r})\right]\right. \\
& +n_{0} \int_{D} \frac{1}{r_{i}^{2}} \mathbf{E}_{i}\left(\mathbf{r}_{i}, \mathbf{R}_{i}\right) \times\left[\widehat{\mathbf{r}}_{i} \times \mathbf{E}_{i}^{\star}\left(\mathbf{r}_{i}, \mathbf{R}_{i}\right)\right] \mathrm{d}^{3} \mathbf{R}_{i} \\
& +n_{0}^{2} \int_{D} \frac{1}{r_{i}^{2} R_{i j}^{2}} \mathbf{E}_{i j}\left(\mathbf{r}_{i}, \mathbf{R}_{i j}, \mathbf{R}_{j}\right) \times\left[\widehat{\mathbf{r}}_{i} \times \mathbf{E}_{i j}^{\star}\left(\mathbf{r}_{i}, \mathbf{R}_{i j}, \mathbf{R}_{j}\right)\right] \\
& \left.\times \mathrm{d}^{3} \mathbf{R}_{j} \mathrm{~d}^{3} \mathbf{R}_{i}+\cdots\right\},
\end{aligned}
$$

so that by means of the vector identity $\mathbf{a} \times\left(\widehat{\mathbf{b}} \times \mathbf{a}^{\star}\right)=|\mathbf{a}|^{2} \widehat{\mathbf{b}}$ for $\mathbf{a}$. $\widehat{\mathbf{b}}=0$, we infer that

$$
\begin{aligned}
\mathbf{S}(\mathbf{r}) & =\frac{1}{2} \sqrt{\frac{\varepsilon_{1}}{\mu_{0}}}\left[\left|\mathbf{E}_{\mathrm{c}}(\mathbf{r})\right|^{2} \widehat{\mathbf{s}}+n_{0} \int_{D} \frac{1}{r_{i}^{2}}\left|\mathbf{E}_{i}\left(\mathbf{r}_{i}, \mathbf{R}_{i}\right)\right|^{2} \widehat{\mathbf{r}}_{i} \mathrm{~d}^{3} \mathbf{R}_{i}\right. \\
& \left.+n_{0}^{2} \int_{D} \frac{1}{r_{i}^{2} R_{i j}^{2}}\left|\mathbf{E}_{i j}\left(\mathbf{r}_{i}, \mathbf{R}_{i j}, \mathbf{R}_{j}\right)\right|^{2} \widehat{\mathbf{r}}_{i} \mathrm{~d}^{3} \mathbf{R}_{j} \mathrm{~d}^{3} \mathbf{R}_{i}+\cdots\right] .
\end{aligned}
$$

From Eqs. (174) and (176), we obtain the following angular spectrum representation for the configuration-averaged Poynting vector in terms of the specific intensity:

$\mathbf{S}(\mathbf{r})=-\int \widehat{\mathbf{p}} I(\mathbf{r},-\widehat{\mathbf{p}}) \mathrm{d}^{2} \widehat{\mathbf{p}}$.

The above derivation shows that to compute the configurationaveraged Poynting vector, we have to solve the vector radiative transfer equation for the direction-dependent specific intensity column vector, and then to integrate the direction-weighted specific intensity over all directions.

\subsection{Scalar radiative transfer equation}

The scalar radiative transfer equation can be obtained from the vector equation by neglecting polarization, that is, by assuming that the light is unpolarized. If in Eq. (159) we apply the transformations

$I_{d}=\left[\begin{array}{c}I_{d} \\ Q_{d} \\ U_{d} \\ V_{d}\end{array}\right] \rightarrow\left[\begin{array}{c}I_{d} \\ 0 \\ 0 \\ 0\end{array}\right]$ and $I_{c}=\left[\begin{array}{c}I_{c} \\ Q_{c} \\ U_{c} \\ V_{c}\end{array}\right] \rightarrow\left[\begin{array}{c}I_{c} \\ 0 \\ 0 \\ 0\end{array}\right]$,

we get a radiative transfer equation for the diffuse specific intensity $I_{d}$ involving the $(1,1)$ element $\bar{Z}_{11}$ of the normalized phase matrix (the phase function). This simplification is used when the medium is illuminated by unpolarized light and only the specific intensity of the multiply scattered light needs to be computed. It is clear that the specific intensities obtained by solving the vector and the scalar radiative transfer equations are different because the equations that they are satisfying are different.

In the last step of our analysis we present a more strict derivation of the scalar radiative transfer equation with the goal of emphasizing the underlying assumptions. To simplify the analysis, we assume that the particles are spherical. The procedure is to obtain representations of the "energetic" quantities $\left|\mathbf{E}_{i}\right|^{2},\left|\mathbf{E}_{i j}\right|^{2}$, etc. appearing in the series (174) for unpolarized light. We begin with some preliminary results characterizing the propagation and scattering of unpolarized light in a discrete random medium consisting of spherical particles. For this purpose, we express the singlescattering field $\mathbf{E}_{i}$ of Eq. (167) as

$\mathbf{E}_{i}\left(\mathbf{r}_{i}, \mathbf{R}_{i}\right)=\overline{\mathbf{t}}\left(\widehat{\mathbf{r}}_{i}, r_{i}\right) \cdot \mathbf{E}_{\mathrm{scti}}^{\infty}\left(\widehat{\mathbf{r}}_{i}\right)$,

with

$\mathbf{E}_{\mathrm{scti} i}^{\infty}\left(\widehat{\mathbf{r}}_{i}\right)=\overline{\mathbf{A}}\left(\widehat{\mathbf{r}}_{i}, \widehat{\mathbf{s}}\right) \cdot \mathbf{E}_{\mathrm{c}}\left(\mathbf{R}_{i}\right)$.

Obviously, we have $\widehat{\mathbf{r}}_{i} \cdot \mathbf{E}_{\mathrm{scti}}^{\infty}\left(\widehat{\mathbf{r}}_{i}\right)=0$, and from $\overline{\mathbf{t}}\left(\widehat{\mathbf{r}}_{i}, 0\right)=\overline{\mathbf{I}}$, we get $\mathbf{E}_{i}\left(0, \mathbf{R}_{i}\right)=\mathbf{E}_{\text {sct }}^{\infty}\left(\widehat{\mathbf{r}}_{i}\right)$.

1. The Stokes column vector $I_{c}$ associated with the coherent field $\mathbf{E}_{\mathrm{c}}$, defined by (cf. Eq. (37)),

$\mathbf{E}_{\mathrm{c}}(\mathbf{r})=\overline{\mathbf{t}}(\widehat{\mathbf{s}}, s(\mathbf{r},-\widehat{\mathbf{s}})) \cdot \mathbf{E}_{0}\left(\mathbf{r}_{A}\right)$

and satisfying $\mathbf{E}_{\mathrm{c}}\left(\mathbf{r}_{A}\right)=\mathbf{E}_{0}\left(\mathbf{r}_{A}\right)$, solves the differential equation (cf. Eq. (57))

$$
\frac{\mathrm{d}_{\mathrm{c}}(\mathbf{r})}{\mathrm{d} s}=-n_{0} C_{\mathrm{ext}} \mathrm{I}_{\mathrm{c}}(\mathbf{r}),
$$

where $s=s(\mathbf{r},-\widehat{\mathbf{s}})$ and $C_{\text {ext }}$ is the extinction cross section. In (181) we used the fact that for spherical particles, the extinction matrix is $\mathrm{K}=C_{\text {ext }} \mathrm{I}_{4}$, where $\mathrm{I}_{4}$ is the $4 \times 4$ identity matrix ( $\mathrm{K}$ is diagonal and does not depend on the incidence direction). Taking into account the similarity between Eqs. (178) and (180), we deduce that the specific intensity column vector $\mathrm{I}_{i}=\mathrm{I}_{i}\left(\mathbf{r}_{i}\right)$ associated with $\mathbf{E}_{i}=\mathbf{E}_{i}\left(\mathbf{r}_{i}, \mathbf{R}_{i}\right)$, i.e.,

$\mathrm{I}_{i}=\frac{1}{2} \sqrt{\frac{\varepsilon_{1}}{\mu_{0}}}\left[\begin{array}{c}\left|E_{i \theta}\right|^{2}+\left|E_{i \varphi}\right|^{2} \\ \left|E_{i \theta}\right|^{2}-\left|E_{i \varphi}\right|^{2} \\ -E_{i \theta} E_{i \varphi}^{\star}-E_{i \varphi} E_{i \theta}^{\star} \\ \mathrm{j}\left[\mathrm{E}_{\mathrm{i} \varphi} \mathrm{E}_{\mathrm{i} \theta}^{\star}-\mathrm{E}_{\mathrm{i} \theta} \mathrm{E}_{\mathrm{i} \varphi}^{\star}\right]\end{array}\right]$,

satisfies the differential equation

$\frac{\mathrm{dI}_{i}\left(\mathbf{r}_{i}\right)}{\mathrm{d} r_{i}}=-n_{0} C_{\text {ext }} \mathrm{I}_{i}\left(\mathbf{r}_{i}\right)$.

For the first component of $I_{i}$,

$I_{i}\left(\mathbf{r}_{i}\right)=\frac{1}{2} \sqrt{\frac{\varepsilon_{1}}{\mu_{0}}}\left|\mathbf{E}_{i}\left(\mathbf{r}_{i}, \mathbf{R}_{i}\right)\right|^{2}$,

the differential equation (182) gives

$\frac{\mathrm{d} I_{i}\left(\mathbf{r}_{i}\right)}{\mathrm{d} r_{i}}=-n_{0} C_{\text {ext }} I_{i}\left(\mathbf{r}_{i}\right)$.

The solution to Eq. (184) is

$I_{i}\left(\mathbf{r}_{i}\right)=\mathrm{e}^{-n_{0} C_{\mathrm{ext}} r_{i}} I_{i}(0)$;

whence, by using the initial condition $\left(\mathrm{cf} . \mathbf{E}_{i}\left(0, \mathbf{R}_{i}\right)=\mathbf{E}_{\mathrm{sct}}^{\infty}\left(\widehat{\mathbf{r}}_{i}\right)\right)$

$I_{i}(0)=\frac{1}{2} \sqrt{\frac{\varepsilon_{1}}{\mu_{0}}}\left|\mathbf{E}_{\mathrm{sct}}^{\infty}\left(\widehat{\mathbf{r}}_{i}\right)\right|^{2}$,

we find

$\left|\mathbf{E}_{i}\left(\mathbf{r}_{i}, \mathbf{R}_{i}\right)\right|^{2}=\mathrm{e}^{-n_{0} \mathrm{Cext}_{\mathrm{e}} r_{i}}\left|\mathbf{E}_{\mathrm{sct}}^{\infty}\left(\widehat{\mathbf{r}}_{i}\right)\right|^{2}$,

or equivalently,

$\left|\overline{\mathbf{t}}\left(\widehat{\mathbf{r}}_{i}, r_{i}\right) \cdot \mathbf{E}_{\mathrm{sct}}^{\infty}\left(\widehat{\mathbf{r}}_{i}\right)\right|^{2}=\mathrm{e}^{-n_{0} C_{\text {ext }} r_{i}}\left|\mathbf{E}_{\mathrm{sct}}^{\infty}\left(\widehat{\mathbf{r}}_{i}\right)\right|^{2}$. 
Eq. (188) describes the propagation (attenuation) of the field scattered by the spherical particle $i$ at the observation point through a discrete random medium.

2. For the far-field pattern $\mathbf{E}_{\mathrm{sct} i}^{\infty}\left(\widehat{\mathbf{r}}_{i}\right)$, we have the representation

$\left|\mathbf{E}_{\mathrm{scti}}^{\infty}\left(\widehat{\mathbf{r}}_{i}\right)\right|^{2}=\frac{C_{\mathrm{sct}}}{4 \pi} p\left(\widehat{\mathbf{r}}_{i}, \widehat{\mathbf{s}}\right)\left|\mathbf{E}_{\mathrm{c}}\left(\mathbf{R}_{i}\right)\right|^{2}$,

where $C_{\text {sct }}$ is the scattering cross section and $p\left(\widehat{\mathbf{r}_{i}}, \widehat{\mathbf{s}}\right)$ is the phase function. Using $\mathbf{E}_{\mathrm{sct} i}^{\infty}\left(\widehat{\mathbf{r}}_{i}\right)=\overline{\mathbf{A}}\left(\widehat{\mathbf{r}}_{i}, \widehat{\mathbf{s}}\right) \cdot \mathbf{E}_{\mathrm{c}}\left(\mathbf{R}_{i}\right)$, we express Eq. (189) as

$$
\left|\overline{\mathbf{A}}\left(\widehat{\mathbf{r}}_{i}, \widehat{\mathbf{s}}\right) \cdot \mathbf{E}_{\mathrm{c}}\left(\mathbf{R}_{i}\right)\right|^{2}=\frac{C_{\mathrm{sct}}}{4 \pi} p\left(\widehat{\mathbf{r}}_{i}, \widehat{\mathbf{s}}\right)\left|\mathbf{E}_{\mathrm{c}}\left(\mathbf{R}_{i}\right)\right|^{2}
$$

In general, for polarized light and in terms of the Stokes column vector $I_{\mathrm{c}}\left(\mathbf{R}_{i}\right)=\left[I_{\mathrm{c}}, Q_{\mathrm{c}}, U_{\mathrm{c}}, V_{\mathrm{c}}\right]^{T}$ associated with $\mathbf{E}_{\mathrm{c}}\left(\mathbf{R}_{i}\right)$, the scattering cross section $C_{\text {sct }}(\widehat{\mathbf{s}})$ and the phase function $p\left(\widehat{\mathbf{r}}_{i}, \widehat{\mathbf{s}}\right)$ are given respectively, by [1-3]

$$
\begin{aligned}
C_{\mathrm{sct}}(\widehat{\mathbf{s}}) & =\frac{1}{I_{\mathrm{c}}} \int\left[Z_{11}\left(\widehat{\mathbf{r}_{i}}, \widehat{\mathbf{s}}\right) I_{\mathrm{c}}+Z_{12}\left(\widehat{\mathbf{r}}_{i}, \widehat{\mathbf{s}}\right) Q_{\mathrm{c}}\right. \\
& \left.+Z_{13}\left(\widehat{\mathbf{r}}_{i}, \widehat{\mathbf{s}}\right) U_{\mathrm{c}}+Z_{14}\left(\widehat{\mathbf{r}_{i}}, \widehat{\mathbf{s}}\right) V_{\mathrm{c}}\right] \mathrm{d}^{2} \widehat{\mathbf{r}}_{i},
\end{aligned}
$$

and

$$
\begin{aligned}
p\left(\widehat{\mathbf{r}}_{i}, \widehat{\mathbf{s}}\right) & =\frac{4 \pi}{C_{\mathrm{sct}}(\widehat{\mathbf{s}})} \frac{1}{I_{\mathrm{c}}}\left[Z_{11}\left(\widehat{\mathbf{r}}_{i}, \widehat{\mathbf{s}}\right) I_{\mathrm{c}}+Z_{12}\left(\widehat{\mathbf{r}}_{i}, \widehat{\mathbf{s}}\right) Q_{\mathrm{c}}\right. \\
& \left.+Z_{13}\left(\widehat{\mathbf{r}}_{i}, \widehat{\mathbf{s}}\right) U_{\mathrm{c}}+Z_{14}\left(\widehat{\mathbf{r}}_{i}, \widehat{\mathbf{s}}\right) V_{\mathrm{c}}\right],
\end{aligned}
$$

where $Z_{i j}$ are elements of the phase matrix $z$. For unpolarized light, we have $Q_{c}=U_{c}=V_{c}=0$; hence, Eq. (189) is still valid, but with

$$
C_{\text {sct }}(\widehat{\mathbf{s}})=\int Z_{11}\left(\widehat{\mathbf{r}_{i}}, \widehat{\mathbf{s}}\right) \mathrm{d}^{2} \widehat{\mathbf{r}}_{i},
$$

and

$$
p\left(\widehat{\mathbf{r}}_{i}, \widehat{\mathbf{s}}\right)=\frac{4 \pi}{C_{\mathrm{sct}}(\widehat{\mathbf{s}})} Z_{11}\left(\widehat{\mathbf{r}}_{i}, \widehat{\mathbf{s}}\right) .
$$

If, moreover, the particles are spherical, the scattering cross section does not depend on the incidence direction. Thus, for unpolarized light, Eq. (190) with the phase function as in Eq. (194), describes the scattering by particle $i$ illuminated by the coherent field $\mathbf{E}_{\mathrm{c}}\left(\mathbf{R}_{i}\right)$.

We compute now all terms $\left|\mathbf{E}_{i}\right|^{2},\left|\mathbf{E}_{i j}\right|^{2}$, etc. in the series (174) by means of Eqs. (188) and (190). The assumption that we make is that all scattering processes (e.g., the scattering by particle $j$ when excited by the field coming from i) correspond to unpolarized light. In this case, for the single-scattering field, we have

$$
\left|\mathbf{E}_{i}\left(\mathbf{r}_{i}, \mathbf{R}_{i}\right)\right|^{2}=\mathrm{e}^{-n_{0} C_{\mathrm{ext}} r_{i}} \frac{C_{\mathrm{sct}}}{4 \pi} p\left(\widehat{\mathbf{r}}_{i}, \widehat{\mathbf{s}}\right)\left|\mathbf{E}_{\mathrm{c}}\left(\mathbf{R}_{i}\right)\right|^{2}
$$

while for the double-scattering field, we have

$$
\begin{aligned}
\left|\mathbf{E}_{i j}\left(\mathbf{r}_{i}, \mathbf{R}_{i j}, \mathbf{R}_{j}\right)\right|^{2} & =\mathrm{e}^{-n_{0} C_{\mathrm{ext}} r_{i}} \mathrm{e}^{-n_{0} C_{\mathrm{ext}} R_{i j}}\left(\frac{C_{\mathrm{sct}}}{4 \pi}\right)^{2} \\
& \times p\left(\widehat{\mathbf{r}}_{i}, \widehat{\mathbf{R}}_{i j}\right) p\left(\widehat{\mathbf{R}}_{i j}, \widehat{\mathbf{s}}\right)\left|\mathbf{E}_{\mathrm{c}}\left(\mathbf{R}_{j}\right)\right|^{2} .
\end{aligned}
$$

Then, we find that the specific intensity, given by (cf. Eq. (174))

$$
\begin{aligned}
I(\mathbf{r},-\widehat{\mathbf{p}}) & =\frac{1}{2} \sqrt{\frac{\varepsilon_{1}}{\mu_{0}}}\left[\delta(\widehat{\mathbf{p}}+\widehat{\mathbf{s}})\left|\mathbf{E}_{\mathrm{c}}(\mathbf{r})\right|^{2}\right. \\
& +n_{0} \frac{C_{\mathrm{sct}}}{4 \pi} \int \mathrm{e}^{-n_{0} C_{\mathrm{ext}} p} p(-\widehat{\mathbf{p}}, \widehat{\mathbf{s}})\left|\mathbf{E}_{\mathrm{c}}\left(\mathbf{R}_{i}\right)\right|^{2} \mathrm{~d} p \\
& +n_{0}^{2}\left(\frac{C_{\mathrm{sct}}}{4 \pi}\right)^{2} \int \mathrm{e}^{-n_{0} C_{\mathrm{ext}} p} \mathrm{e}^{-n_{0} C_{\mathrm{ext}} R_{j i}} p\left(-\widehat{\mathbf{p}},-\widehat{\mathbf{R}}_{j i}\right) \\
& \left.\times p\left(-\widehat{\mathbf{R}}_{j i}, \widehat{\mathbf{s}}\right)\left|\mathbf{E}_{\mathrm{c}}\left(\mathbf{R}_{j}\right)\right|^{2} \mathrm{~d} R_{j i} \mathrm{~d}^{2} \widehat{\mathbf{R}}_{j i} \mathrm{~d} p+\cdots\right],
\end{aligned}
$$

solves the integral equation

$$
\begin{aligned}
I(\mathbf{r},-\widehat{\mathbf{p}}) & =\delta(\widehat{\mathbf{p}}+\widehat{\mathbf{s}}) I_{\mathrm{c}}(\mathbf{r}) \\
& +\frac{C_{\mathrm{sct}}}{4 \pi} \int \mathrm{e}^{-n_{0} C_{\mathrm{ext}} p} p\left(-\widehat{\mathbf{p}},-\widehat{\mathbf{p}}^{\prime}\right) I\left(\mathbf{r}+\mathbf{p},-\widehat{\mathbf{p}}^{\prime}\right) \mathrm{d}^{2} \widehat{\mathbf{p}}^{\prime} \mathrm{d} p
\end{aligned}
$$

with

$I_{\mathrm{c}}(\mathbf{r})=\frac{1}{2} \sqrt{\frac{\varepsilon_{1}}{\mu_{0}}}\left|\mathbf{E}_{\mathrm{c}}(\mathbf{r})\right|^{2}$.

The differential form of Eq. (198) gives the scalar radiative transfer equation for the diffuse specific intensity

$I_{\mathrm{d}}(\mathbf{r}, \widehat{\mathbf{q}})=I(\mathbf{r}, \widehat{\mathbf{q}})-\delta(\widehat{\mathbf{q}}-\widehat{\mathbf{s}}) I_{\mathrm{c}}(\mathbf{r})$,

that is,

$$
\begin{aligned}
\frac{\mathrm{d} I_{\mathrm{d}}(\mathbf{r}, \widehat{\mathbf{q}})}{\mathrm{d} s} & =-n_{0} C_{\mathrm{ext}} I_{\mathrm{d}}(\mathbf{r}, \widehat{\mathbf{q}})+\frac{C_{\mathrm{sct}}}{4 \pi} p(\widehat{\mathbf{q}}, \widehat{\mathbf{s}}) I_{\mathrm{c}}(\mathbf{r}) \\
& +\frac{C_{\mathrm{sct}}}{4 \pi} \int p\left(\widehat{\mathbf{q}}, \widehat{\mathbf{q}}^{\prime}\right) I_{\mathrm{d}}\left(\mathbf{r}, \widehat{\mathbf{q}}^{\prime}\right) \mathrm{d}^{2} \widehat{\mathbf{q}}^{\prime},
\end{aligned}
$$

where $p\left(\widehat{\mathbf{q}}, \widehat{\mathbf{q}}^{\prime}\right)=\left(4 \pi / C_{\text {sct }}\right) Z_{11}\left(\widehat{\mathbf{q}}, \widehat{\mathbf{q}}^{\prime}\right)$ (the $(1,1)$ element of the normalized phase matrix).

\section{Conclusions}

The vector radiative transfer theory has been derived for a discrete random layer with non-scattering boundaries and a sparse concentration of particles by using the far-field Foldy equations. The sequential steps of the derivation are summarized below.

1. By means of the far-field approximation, we derived the farfield Foldy equations for the total field and the exciting fields.

2. Considering an iterated solution of the exciting fields equation and employing the Twersky approximation, we obtained an order-of-scattering expansion for the total field. In the Twersky approximation, all scattering paths involving a particle two or more times are neglected.

3. By averaging the Twersky expansion for the total field under the assumption that the positions of the particles are uncorrelated, we computed the coherent field. We discussed several topics related to this issue as for example, the cases of inhomogeneous media and multiple species of particles, the continuous extension of the far-field representation to the near field, the Foldy approximation, and the Foldy integral equation for the coherent field.

4. We computed the coherency dyadic under the ladder approximation, that is, in a Feynman diagram representation, the diagrams with crossing connectors having been neglected. For reasons of clarification, we presented an analytical proof of the ladder approximation. From the ladder expansion, we derived an integral equation for the diffuse specific coherency dyadic, defined through an angular spectrum representation of the coherency dyadic. This integral equation gives the vector radiative transfer equation for the diffuse specific intensity column vector. Finally, we deduced the angular spectrum representations for the Poynting-Stokes dyadic and the configuration-averaged Poynting vector in terms of the specific intensity column vector and the specific intensity, respectively, and derived the scalar radiative transfer equation.

In summary, the main assumptions and approximations of the solution method are (i) the assumption that the positions of the particles are statistically independent (including the assumption that the particles are uniformly distributed), (ii) the far-field approximation, (iii) the Twersky approximation for the total field, and (iv) the ladder approximation for the coherency dyadic. The Twersky approximation as well as the ladder approximation are valid if the number of particles $N$ is very large. 


\section{References}

[1] Mishchenko MI. Vector radiative transfer equation for arbitrarily shaped and arbitrarily oriented particles: a microphysical derivation from statistical electromagnetics. Appl Opt 2002:41:7114-34.

[2] Mishchenko MI, Travis LD, Lacis AA. Multiple scattering of light by particles: radiative transfer and coherent backscattering. Cambridge: Cambridge University Press; 2006

[3] Mishchenko MI. Electromagnetic scattering by particles and particle groups: an introduction. Cambridge, UK: Cambridge University Press; 2014. https://www. giss.nasa.gov/staff/mmishchenko/publications/Book_4.pdf

[4] Mishchenko MI. Directional radiometry and radiative transfer: the convoluted path from centuries-old phenomenology to physical optics. J Quant Spectrosc Radiat Transfer 2014;146:4-33.

[5] Mishchenko MI, Dlugach JM, Yurkin MA, Bi L, Cairns B, Liu L, Panetta RL, Travis LD, Yang P, Zakharova NT. First-principles modeling of electromagnetic scattering by discrete and discretely heterogeneous random media. Phys Rep 2016;632:1-75.

[6] Dyson FJ. Why is maxwell's theory so hard to understand?http://www. clerkmaxwellfoundation.org/DysonFreemanArticle.pdf.

[7] Truesdell C. Ergodic theory in classical statistical mechanics. New York: Ergodic Theories, Academic Press; 1961. p. 21-56.
[8] Twersky V. On propagation in random media of discrete scatterers. Proc Symp Appl Math 1964;16:84-116.

[9] Saxon DS. Lectures on the scattering of light. Science report no. 9. Log Angeles: Department of Meteorology, Unviversity of California; 1955.

[10] Waterman PC, Truell R. Multiple scattering of waves. J Math Phys $1961 ; 2: 512-37$.

[11] Foldy LL. The multiple scattering of waves. Phys Rev 1945;67:107-19.

[12] Ishimaru A. Wave propagation and scattering in random media. New York: Academic Press; 1978. (also IEEE Press-Oxford University Press Classic Reissue; 1997)

[13] Feynman RP. Space-time approach to nonrelativistic quantum mechanics. Rev Mod Phys 1948;20:367-87.

[14] Bourret RC. Propagation of randomly perturbed fields. Can J Phys 1962;40:782-90.

[15] Furutsu K. On the statistical theory of electromagnetic waves in a fluctuating medium. J Res Natl Bur Stand D 1963;67:303-23.

[16] Tatarski VI. Wave propagation in a turbulent medium. New York: McGraw-Hill; 1961.

[17] Frisch U. Wave propagation in random media. In: Bharucha-Reid AT, editor. Probabilistic methods in applied mathematics. New York: Academic Press; 1968. p. 75-198.

[18] Mishchenko MI. Poynting-stokes tensor and radiative transfer in discrete random media: the microphysical paradigm. Opt Express 2010;18:19771-91. 